Portland State University

PDXScholar

Dissertations and Theses

Dissertations and Theses

Spring 6-10-2019

\title{
Sensors and Portable Instruments for Postharvest Agriculture
}

Ryan M. Lerud

Portland State University

Follow this and additional works at: https://pdxscholar.library.pdx.edu/open_access_etds

Part of the Food Science Commons, and the Physics Commons Let us know how access to this document benefits you.

Recommended Citation

Lerud, Ryan M., "Sensors and Portable Instruments for Postharvest Agriculture" (2019). Dissertations and Theses. Paper 4994.

https://doi.org/10.15760/etd.6870

This Dissertation is brought to you for free and open access. It has been accepted for inclusion in Dissertations and Theses by an authorized administrator of PDXScholar. Please contact us if we can make this document more accessible: pdxscholar@pdx.edu. 
Sensors and Portable Instruments for Postharvest Agriculture

\author{
by
}

Ryan M. Lerud

A dissertation submitted in partial fulfillment of the requirements for the degree of

Doctor of Philosophy

in

Applied Physics

Dissertation Committee:

Raj Solanki, Chair

Shankar Rananavare, Co-Chair

Andrew Rice

Robert Bass

Portland State University

2019 


\section{Abstract}

The sensing needs for the fresh produce industry can be split into two primary stages: during maturation in the field, also referred to as Precision Farming, and during storage and transport of the produce, or Postharvest Storage. This work seeks to improve the accuracy and reliability of commercially available electrochemical and spectroscopic sensors tailored to the sensing needs of the fresh produce industry. For electrochemical sensing, this study proposes the use of an inline filter to remove polar organic compounds, which can interfere with the readings of a platinum-based electrochemical sensor. A 50\% improvement in measurement accuracy was achieved when monitoring the storage headspace of a container of apples. For portable spectroscopy instruments, this study suggests improvements for the alignment of the optical bench and the spectral collect protocol. Methods to reduce the influence of environmental noise, such as variability of background light (sunlight in the field) and thermal effects on hardware performance, are presented. This study also presents the first report of the calibration transfer of spectral regressions developed with Karl Norris's Derivative Quotient Method. The motivation for this aspect of research was to develop methods to collect stable and accurate data in the field, which can be used to improve the quality of fresh produce reaching the customer and reduce premature food spoilage. 


\section{Acknowledgements}

I would like to extend my sincerest thanks and appreciation to the people who helped me on this journey.

My first acknowledgement must go to Dr. Peter Moeck, for picking me up out of the mud during my undergrad and setting me on this path. I would like to also acknowledge Dr Shankar Rananavare for the freedom to find my own path during my graduate studies.

I would like to express my gratitude to Dr. Kerry Walsh and Dr. Phul Subedi of Central Queensland University for their mentorship and friendship over the years. I can say whole heartily that both of you help me grow as a Scientist and a Human. Mr. Gary Ritchie deserves a great deal of recognition for pushing me to be a better writer and giving me the opportunity to build my professional brand in the international near infrared spectroscopy community and encouraging me to serve as elected delegate-atlarge for the Council for Near Infrared Spectroscopy (CNIRS).

I would also like to extend my thanks to my brother Philip Lerud, my lab mates, and my coworkers for their assistance and support over the years. Finally, a special thank you to Dr. Raj Solanki for helping me see this journey through to the end. 
Table of Contents

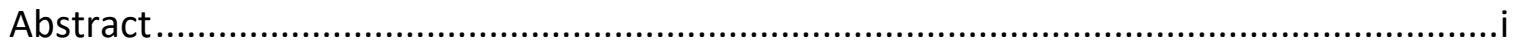

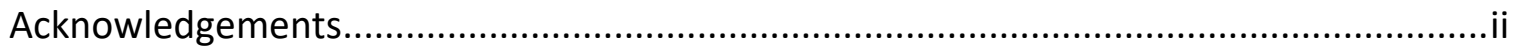

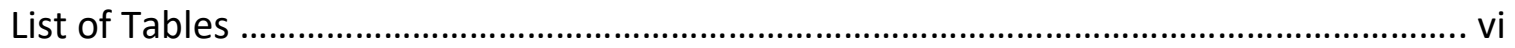

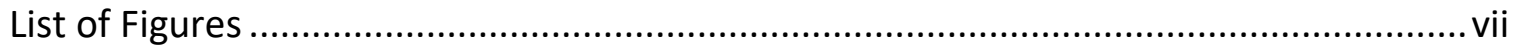

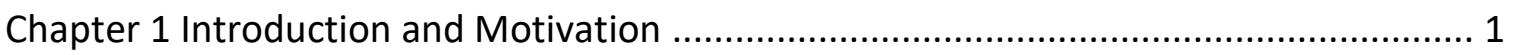

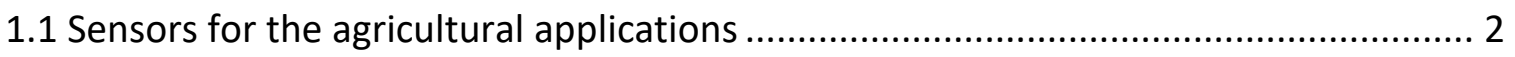

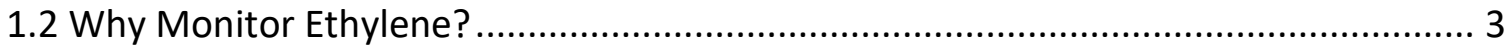

1.3 Current Methods for Ethylene Detection ........................................................... 4

1.4 Theory of Operation Electrochemical Ethylene Sensor ......................................... 4

1.5 Theoretical Performance Criteria for Electrochemical Detection of Ethylene Gas ...... 6

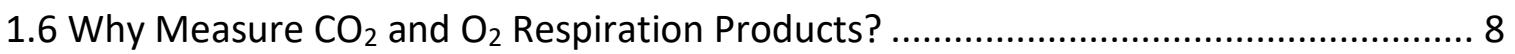

1.7 Why use Handheld Reflectance NIR Spectroscopy to Monitor Fruit Maturity? .......... 8

1.8 Combining the Two Sensing Platforms to Optimize Fruit Quality ............................. 8

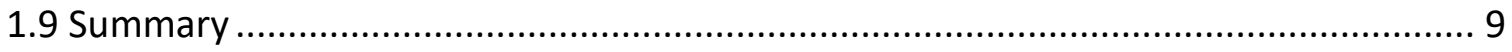

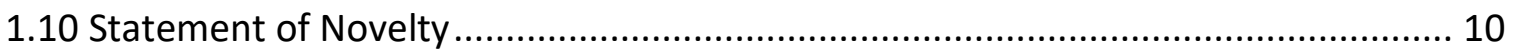

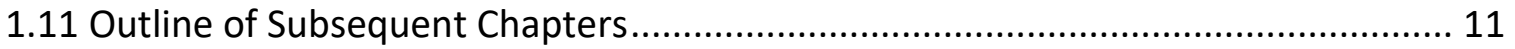

Chapter 2 Optimizing the Performance of a Commercial Electrochemical Ethylene Sensor via Controlled Ethylene Generation in Situ...................................................... 12

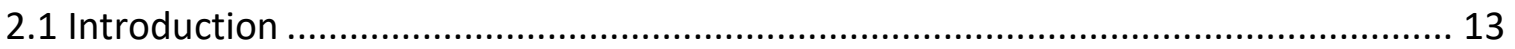

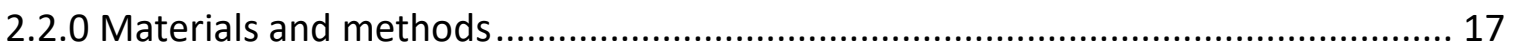

2.2.1 Preparation of Ethephon $\mathrm{KCl}$ Powder......................................................... 17

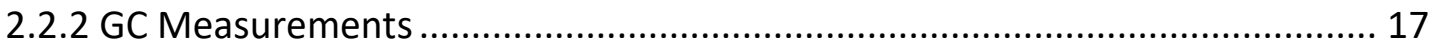

2.2.3 Startup procedure of F-900 for Ethephon Headspace ................................. 18

2.2.4 Preparation of Calibration Standards using Ethephon .................................. 18

2.2.5 Measurement of Ethylene Released by Ethephon Reaction........................... 18

2.2.6 Measurements of Ethylene Production from Apple ...................................... 20

2.2.7 Validation of Reduction of Volatile Organic Compounds in Airstream ............ 22

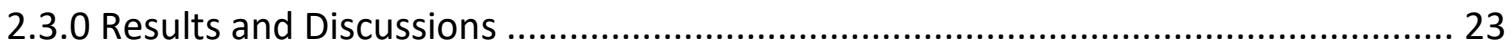




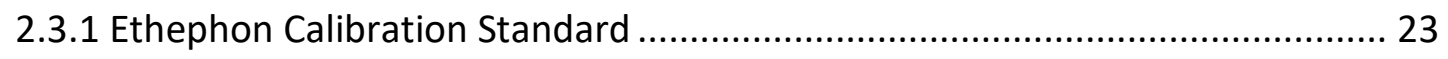

2.3.2 Operational principle for polar volatile organic compounds trap ................... 26

2.3.3 Theory of regenerating the trap ................................................................. 27

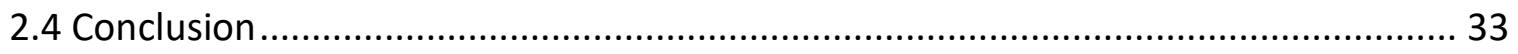

Chapter 3 Handheld diode array spectrometer for maturity estimations in thin-skinned

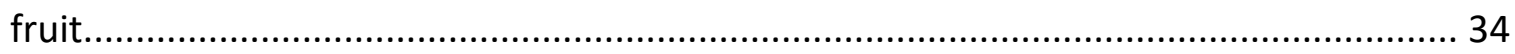

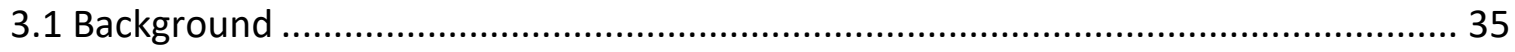

3.1.2 Background on Multivariate Math .............................................................. 37

3.1.3 The Importance of Reference Method Accuracy In PLSR................................. 39

3.1.4 Practical Constraints on Sampling and Population Selection for PLSR in

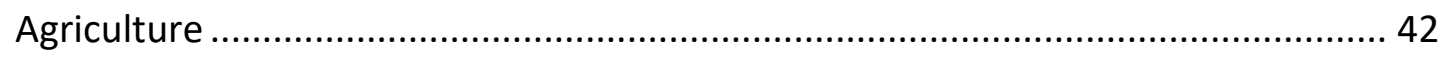

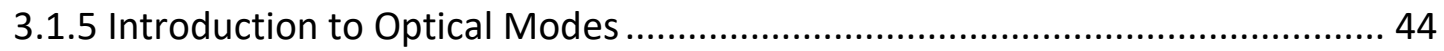

3.1.6 Types of Reflectance Rays from a Sample..................................................... 45

3.1.7 Why use Shortwave Near Infrared Spectroscopy? .......................................... 47

3.1.8 Experiment for Approximation of Penetration Depth into Cantaloupe .......... 48

3.2 The Influence of Dynamic Environmental Factors...................................................... 50

3.3 Optimizing the sample probe distance ................................................................. 51

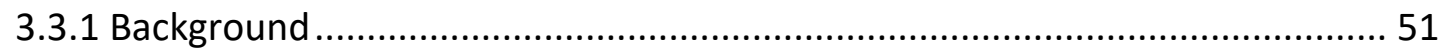

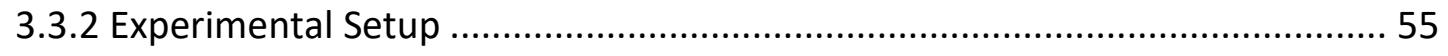

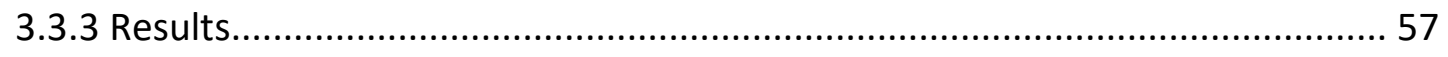

3.3.4 Conclusion of the collimating tube experiment ............................................ 59

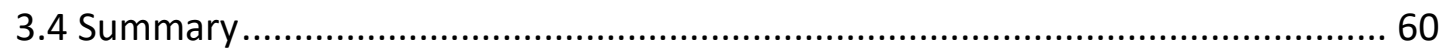

Chapter 4 The Role of Spectrum Collection Protocols and Reference Scans ................... 62

4.1 Overview of Design Considerations for Handheld Spectrometers ............................. 63

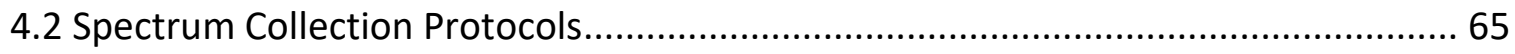

4.3 Thermal Effects on The Illumination Source ............................................................... 66

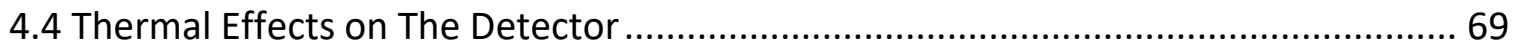

4.5 Two Methods for White Tile Reference................................................................ 71

4.6 Comparison of Thompson grape calibration for an Internal on-demand vs External White Reference Target spectrometer system under Ideal Conditions.......................... 72 
4.7 External White Reference Target under Ideal and Non-ideal Conditions ................. 74

4.8 Internal White Reference Target under Ideal and Non-ideal Conditions.................. 76

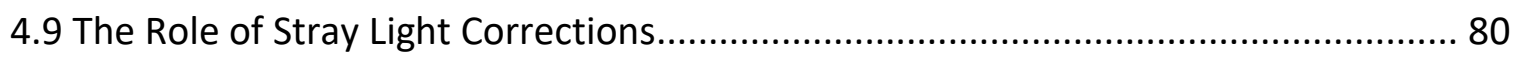

4.9.1 SCiO Spectrometer Susceptibility to External Light ...................................... 80

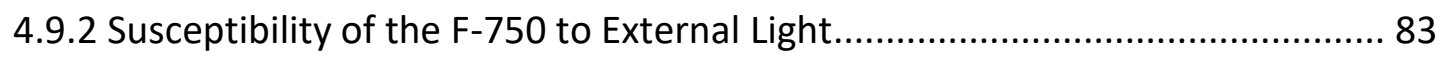

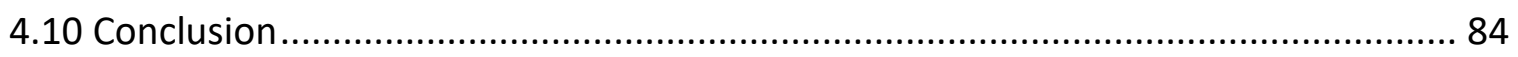

Chapter 5 Comparison of Derivative Quotient Method and PLSR for SWNIRS model

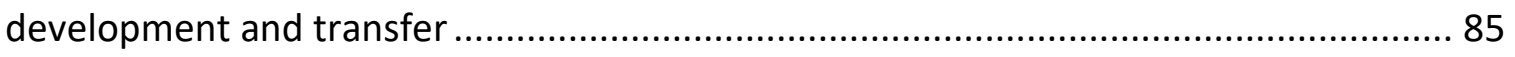

5.1 Evaluation of the Derivative Quotient Method For Spectral Regression .................. 86

5.2 Understanding how the Gap-Smoothing Convolution Function is used by DQM ...... 89

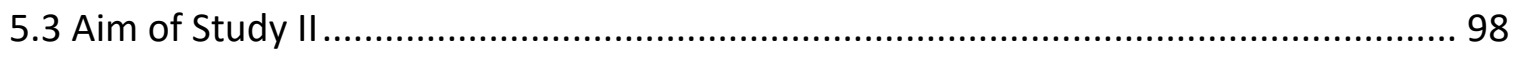

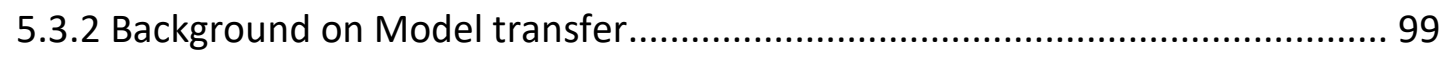

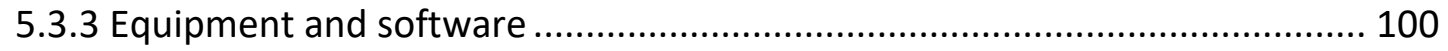

5.3.4 Exercise 1 - Comparison of DQM and PLSR for sugar water mixtures .......... 102

5.3.5 Exercise 2 - Direct calibration transfer of sugar water models .................... 104

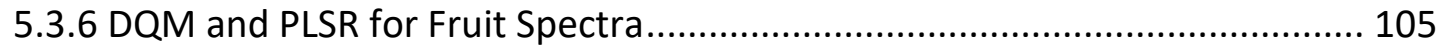

5.3.7 Exercise 3 How the number of terms influence a single instrument DQM calibration For DMC of Mango ......................................................................... 108

5.3.8 Exercise 4 Mixing First and Second Derivative Terms ................................. 110

5.3.9 Exercise 5 Development of a Global 6 instrument DQM Calibration ............. 111

5.3.10 Exercise 6 - Calibration transfer of PLSR and DQM models for mangos ...... 113

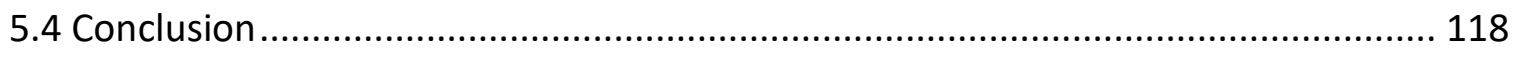

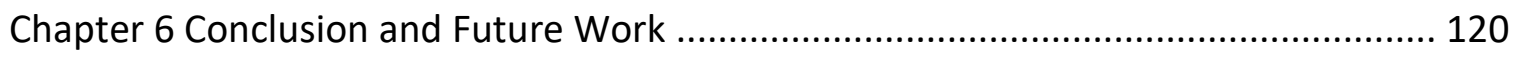

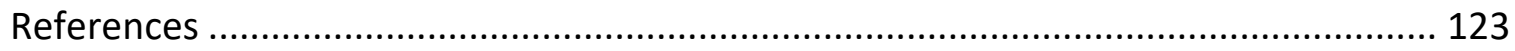

Appendix A - Supplemental Information Chapter 2 .............................................. 131

Appendix B - Calibration transfer between short wave near infrared photodiode array

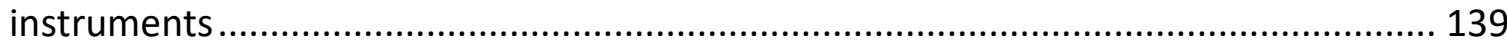


List of Tables

Table 1.1. Performance statistics for a typical electrochemical sensor from Interscan when exposed to dry nitrogen and ethylene gas standards. ......................................... 7

Table 2.1. Dependence of ethylene concentration on ethephon concentration. ............ 26

Table 3.1. Comparison of sugar water concentration (measured in ${ }^{\circ} \mathrm{Brix}$ ) reference

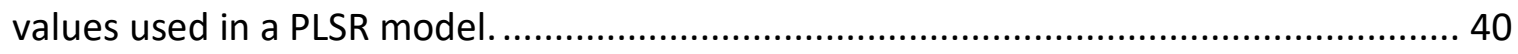

Table 4.1. Contrasts PLSR statistics for two shortwave-NIR handheld spectrometers... 73

Table 4.2. Comparison of pixel values for dark current correction in the F-750............. 78

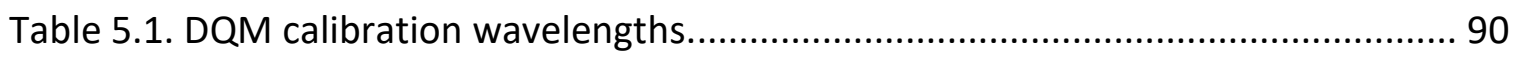

Table 5.2. Wavelength range / parameters used for PLSR and DQM models. ................ 103

Table 5.3. Prediction model validation results for sugar water (Brix) models. Exercise 1

Table 5.4. Performance of the master instrument...................................................... 107

Table 5.5. Influence of the number of terms for a 1st derivative calibration. ............... 108

Table 5.6. Influence of the number of terms for a 2 nd derivative calibration............... 109

Table 5.7. Influence of the number of terms for a 1st derivative calibration with gap limit

9. 110

Table 5.8. 2-term Second Derivative Numerator, First Derivative Denominator, gap limit

7.

Table 5.9. 2-term First Derivative Numerator, Second Derivative Denominator, gap limit

7.

Table 5.10. Influence of the number of terms for Global $1^{\text {st }}$ derivative calibration for

DMC.

Table 5.11. Influence of the number of terms for an Global $1^{\text {st }}$ derivative calibration for

DMC.

Table 5.12. DQM vs PLS calibration transfer results. $R^{2}$

Table 5.13. DQM vs PLS calibration transfer results. Bias 118 


\section{List Of Figures}

Figure 1.1. Diagram of Electrochemical Sensor Photo of an actual electrochemical sensor

Figure 2.1. Block diagram of the external setup for ethephon reaction ........................ 20

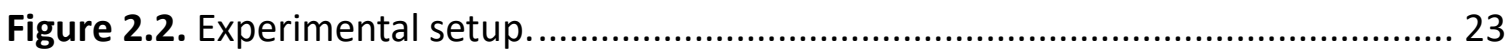

Figure 2.3. Electrochemical response as recorded by the F-900 for a standard calibration gas and ethephon generated ethylene ..................................................................... 25

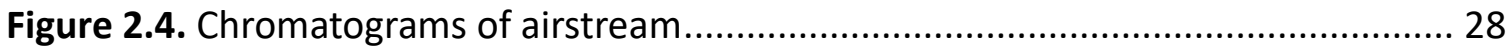

Figure 2.5. Continuous measurement of apple headspace........................................ 29

Figure 2.6. Six repeated measurements and regeneration cycles (0-100 minutes) of apple

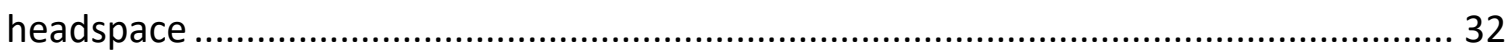

Figure 3.1. Determining the number of principle components selected ......................... 39

Figure 3.2. Interpolated 9 point Savitzky-Golay second derivative transflection spectra of sugar water

Figure 3.3. Predicted vs Reference graph for A) the estimated ${ }^{\circ}$ Brix concentration B) Actual ${ }^{\circ}$ Brix concentration reference values. ................................................................. 41

Figure 3.4. Optical modes used in NIR Spectroscopy of Fresh Produce......................... 44 Figure 3.5. Specular reflection, shown in blue, and diffuse reflection, shown in green,

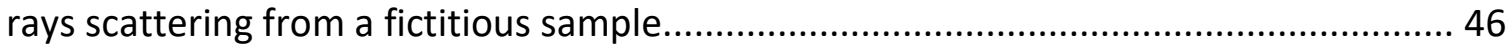

Figure 3.6. Interactance spectra path or a body transflection path ............................... 46

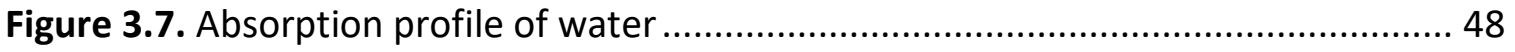

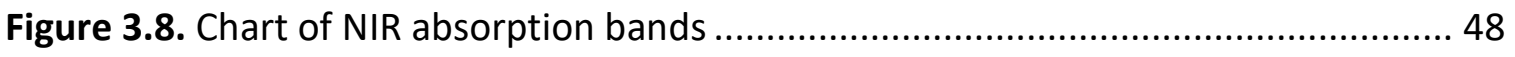

Figure 3.9. Experimental setup for the qualitative measurement of path length .......... 49

Figure 3.10. Cantaloupe spectra based on pathlength ............................................ 50

Figure 3.11. The prototype shadow probe spectrometer collecting sugar water spectra.

Figure 3.12. Contrasting the performance for a sugar water transflection experiment, which is thought to be a first approximation of an apple, and an apple dry matter

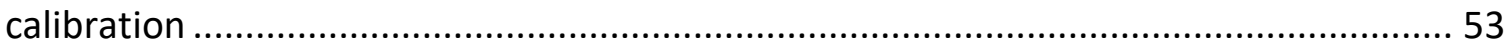

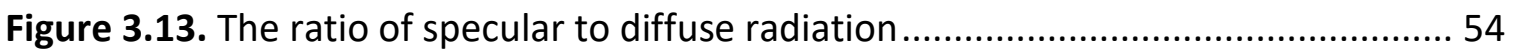

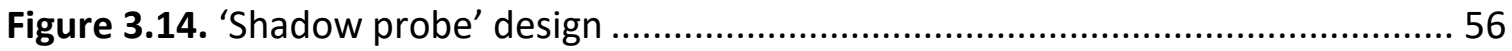

Figure 3.15. Photo of the experimental setup used to measure the influence of probe to

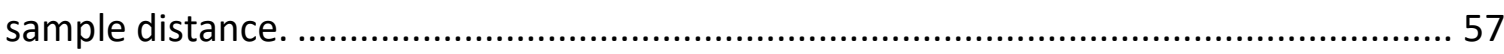

Figure 3.16. The visual shadow cast by the probe onto the white tile. .......................... 58 Figure 3.17. The Reflectance ratio of Teflon to ceramic tile as a function of distance and tube finish 59

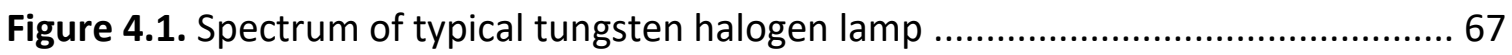


Figure 4.2. Relative intensity of a xenon tungsten lamp collected with a Zeiss MMS-1.. 68

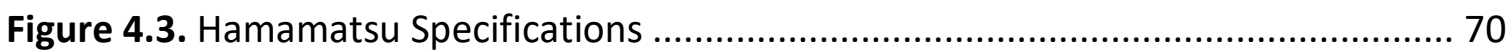

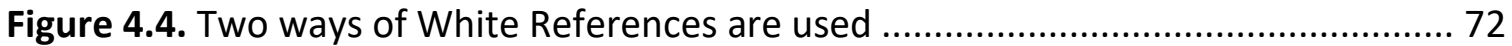

Figure 4.5. Sample presentation of a Red Thompson grape ........................................ 73

Figure 4.6. Standard deviation for 20 scans of PTFE as collected by the SCiO. ............... 75

Figure 4.7. Influence of temperature on the spectra collected by the SCiO spectrometer

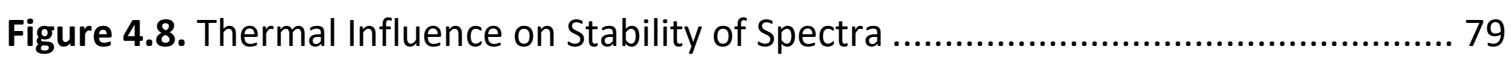

Figure 4.9. Standard Deviation of 20 repeated scans of an external thermally stable PTFE

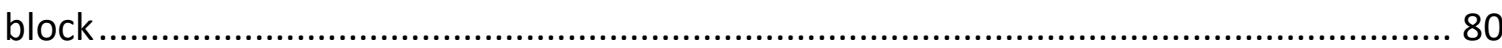

Figure 4.10. Influence of Stray light on the second derivative spectra collected by the

SCiO Spectrometer ................................................................................................. 81

Figure 4.11. Spectra of a Thompson table grape under external lighting....................... 83

Figure 5.1. Specta for first term numerator .............................................................. 91

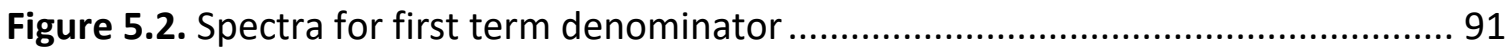

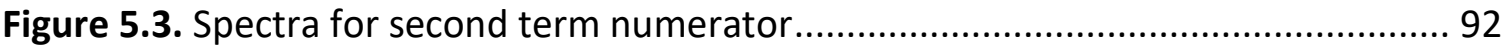

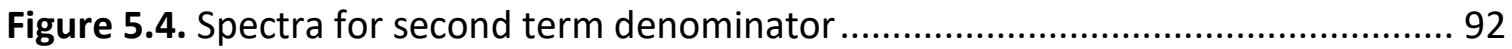

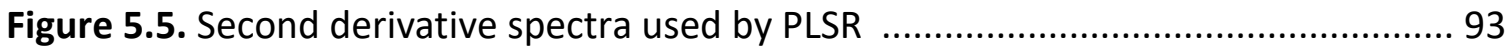

Figure 5.6. The convolution function for each for the numerator and denominator terms

95

Figure 5.7. PLS Regression Vector for Sugar Water..................................................... 96

Figure 5.8. Correlation between first term numerator and sugar water...................... 97

Figure 5.9. Correlation between first term denominator and sugar water................... 98 


\section{Chapter 1}

Introduction and Motivation 


\subsection{Sensors for the agricultural applications}

The fresh fruit business is a several hundred billion dollar market. According to the US Apple commission, US farmers sell roughly \$4 billion worth of apples per year, with a projected additional $\$ 15$ billion of related downstream economic activity ${ }^{1}$. These farmers, along with their downstream partners, rely on various sensors starting with detection of physiological responses over a plant's life cycle ${ }^{2}$. The sensing needs for the fresh produce industry can be split into two primary stages: during maturation in the field, also referred to as Precision Farming, and during storage and transport of the produce, or Postharvest Storage.

The objective of the conducted research was to address limitations in sensing for precision farming and postharvest storage via two different, but complementary methods. The first method involves the development of an inline gas filter and ondemand calibration source for a compact electrochemical sensor to detect ethylene gas. Ethylene gas is the plant hormone that causes green bananas to ripen and turn yellow. The second method uses a near-infrared reflectance-based spectrometer that infers internal quality factors of thin-skinned fruit. The electrochemical sensor should allow detection of ethylene at $\mathrm{nL} / \mathrm{L}$ levels. Use of a reflectance spectrometer for fruit ripening application involves analysis and machine learning to determine fruit ripening stages and internal quality in a matter of seconds ${ }^{3}$. The limitations of spectroscopy addressed in this research include bias when transferring calibrations between instruments, the 
influence of environmental factors on hardware performance and repeatability, and the role and required frequency of a white reference spectra. These two sensing systems can be combined to deliver information that correlates with industry standards for supply chain management of fresh produce.

\subsection{Why Monitor Ethylene?}

One physiological response of interest is that of ethylene $\left(\mathrm{C}_{2} \mathrm{H}_{4}\right)$, a classical plant hormone associated with the ripening processes in climacteric fruit. The classification of climacteric is a stage of fruit ripening associated with increased ethylene production and a rise in cellular respiration ${ }^{4}$. Fruit that do not respond to ethylene are classified as nonclimacteric. As the trend of global trade in fresh fruit increases, there is a need to monitor and control ethylene levels during transportation to ensure fruit quality is maintenaned during long term storage ${ }^{5}$. The Postharvest Department at UC Davis reports that 'Gala' apples are optimally stored at $0{ }^{\circ} \mathrm{C}$ under 90 to $95 \%$ relative humidity. Under these conditions Gala apples will respire ethylene gas at a rate of 4 to $12 \mu \mathrm{L} / \mathrm{Kg} \cdot \mathrm{hr} .{ }^{6}$ Assuming a 100 ton airtight storage room has a volume of $300 \mathrm{~m}^{3}$ and holds $3001 \mathrm{~m}^{3}$ containers of apples $^{7}$, then each container stores approximately $300 \mathrm{~kg}$ of apples. If an $80 \%$ packing density is also assumed for each container and any space between the containers is ignored, then apples occupies $240 \mathrm{~m}^{3}$ of the storage volume and the remaining $60 \mathrm{~m}^{3}$ is airspace for the external accumulation of ethylene gas. Working backwards from the respiration rate of ethylene, the storage room will have a buildup of 6 to $18 \mu \mathrm{L} / \mathrm{hr}$ of 
ethylene gas. After 24 hours of storage the expected ethylene concentration would be over 1000 times greater than the half-maximal physiological response for ethylene of 0.1 $\mu \mathrm{L} / \mathrm{L}$ in the $a \mathrm{ir}^{8}$. The negative effects of ethylene on apples is further illustrated by the work of Johnston et al. ${ }^{9}$, which found that Gala apples exposed to $0.1-10 \mu \mathrm{L} / \mathrm{L}$ of ethylene over 14 day storage at $20^{\circ} \mathrm{C}$ will result in a drop of firmness from $52+/-3 \mathrm{~N}$ to $22+/-3 \mathrm{~N}$. A decrease in firmness, or softening, is an indicator of lower shelf life and decreases consumer preference for apples ${ }^{10}$. As such, the management of ethylene, offers a method to extend the postharvest life of horticultural produce during storage ${ }^{11}$. The current price per sensor, sensor maintenance, and reliability of ethylene sensing equipment has prevented widespread adoption of in-transit monitoring.

\subsection{Current Methods for Ethylene Detection}

Traditionally, ethylene levels have been measured via electrochemical sensors, gas chromatography-flame ionization detectors (GC-FID), or ring down spectroscopy ${ }^{12,13}$. For rapid and inexpensive measurements of ethylene, electrochemical sensors are ideal. However, electrochemical sensors have issues with stability and cross-sensitivity. The issue of cross-sensitivity is further complicated by the presence of polar volatile organic compounds, which are naturally emitted by the fruit ${ }^{14}$.

\subsection{Theory of Operation Electrochemical Ethylene Sensor}

A basic amperometric electrochemical sensor is comprised of at least two electrodes held at a fixed voltage. The working electrodes typically are made of platinum or 
gold $^{15-17}$. As shown in Figure 1.1, when the analyte airstream is passed over the sensor, a portion of the molecules diffuse through a membrane and are oxidized at the air-metal interface on a working electrode. The current change generated by the oxidation of gas molecules is then measured by an electrical circuit and converted to a concentration of ethylene. Equation 1 shows that 12 electrons are released for every molecule of ethylene that is completely oxidized at the working electrode.

$$
\mathrm{C}_{2} \mathrm{H}_{4(\mathrm{gas})}+4 \mathrm{H}_{2} \mathrm{O}+\mathrm{Pt} \rightarrow \mathrm{Pt}+2 \mathrm{CO}_{2}+12 \mathrm{H}^{+}+12 e^{-}
$$
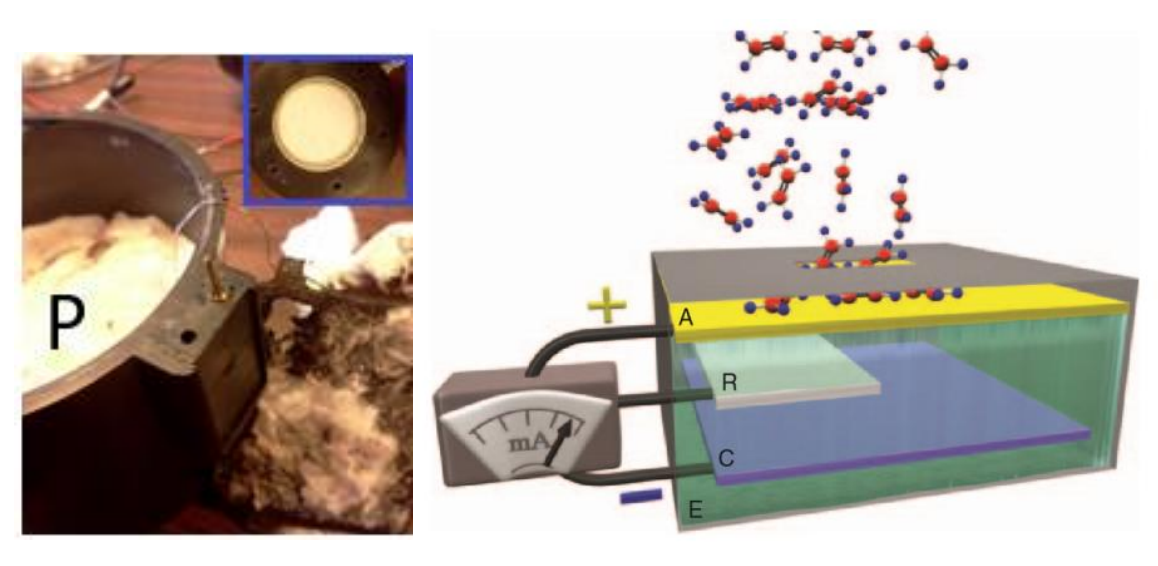

Figure 1.1. Diagram of Electrochemical Sensor P) Photo of an actual electrochemical sensor $B)$ Schematic of an electrochemical sensor. Ethylene diffuses through a barrier into the sensor, which consists of a sensing electrode (anode, A), a counter electrode (cathode, C) and a reference electrode $(R)$ covered by a thin layer of an electrolytic solution (E). If an electrical potential is applied to the anode ethylene is catalytically oxidized, resulting in a current change proportional to the ethylene concentration ${ }^{13}$.

Platinum-based electrochemical sensors are known to react with multiple hydrocarbon compounds. Cross-sensitivity occurs when more than one species of gaseous molecule 
reacts at the working electrode's surface ${ }^{18,19}$. The current change due to oxidation of any non-ethylene molecules is then measured along with the current change due to the oxidation of ethylene molecules by the electrical circuit and is included in the determination of ethylene concentration. This lack of selectivity results in an artificially high estimate of ethylene concentration, which is especially problematic when attempting to measure ethylene with an expected concentration of $<0.1 \mu \mathrm{L} / \mathrm{L}$ in a complex sample, such as the headspace of a chamber containing apples ${ }^{20,21}$. Gas chromatography can solve the problem of cross sensitivity by use of a column to separate gaseous species before the sensor detection. But, chromatography is not well suited for real-time monitoring.

\subsection{Theoretical Performance Criteria for Electrochemical Detection of Ethylene Gas}

Selectivity, stability, and sensitivity are three important criteria for evaluation of an electrochemical sensor. Work on a prefilter to counteract the poor selectivity of electrochemical sensors for ethylene gas is presented in Chapter 2. The topic of stability has been addressed by the sensor manufacturer Membrapore (France), which offers an electrochemical sensor that has a linear $\sim 2 \%$ loss of sensitivity per month ${ }^{19}$. To further examine the issue of sensitivity, an electrochemical sensor manufactured by Interscan (California, USA) has been evaluated. The sensitivity has units of Analog to Digital Convertor counts ( ADC counts) per $0.001 \mu \mathrm{L} / \mathrm{L}$ of ethylene. The sensitivity and lower detection limit are calculated by equations 2 and 3. Performance statistics for a typical electrochemical sensor from Interscan when exposed to dry nitrogen and ethylene gas standards is presented in Table 1.1. 
Sensitivity $=A D C$ Counts $/ \mathrm{nL}^{-1}=[(60$ Second Average of Stabile Ethylene Response $)-(60$ Second Average of Background "Zero" Response)] / (Concentration of Ethylene Gas nL/L) [2]

Lower Detection Limit $(1 \sigma)=$ Standard Deviation of Zero Gas (60 second interval) X Sensitivity [3]

\begin{tabular}{|l|r|l|}
\hline \multicolumn{3}{|c|}{ Calculations for a Typical Interscan Sensor } \\
\hline Average: Background Zero & 2084010 & ADC counts \\
\hline Average: Gas Stream & 2868165 & ADC counts \\
\hline Signal Response & 784155 & ADC counts \\
\hline Std Counts: Background Zero & 9096 & ADC counts \\
\hline Std Counts: Gas Stream & 8985 & ADC counts \\
\hline Gas Concentration & 1600 & $\mathrm{~nL} / \mathrm{L}$ \\
\hline Sensitivity & 504 & ADC counts/(nL/L) \\
\hline Signal To Noise Ratio & 86 & \\
\hline Noise: Background Zero & 18 & $\mathrm{~nL} / \mathrm{L}$ \\
\hline Noise: Gas Stream & 17.8 & $\mathrm{~nL} / \mathrm{L}$ \\
\hline Lower Detection Limit & 54.1 & $\mathrm{~nL} / \mathrm{L}$ \\
\hline
\end{tabular}

Table 1.1. Performance statistics for a typical electrochemical sensor from Interscan when exposed to dry nitrogen and ethylene gas standards.

If it is assumed that the lower detection limit of a sensor should be below the half-

maximal response for most physiological ethylene effects, then the implied upper limit for minimal detection is $0.1 \mu \mathrm{L} / \mathrm{L}$ in air $^{8}$. By selecting a target lower detection limit, for which three sigma is $0.05 \mu \mathrm{L} / \mathrm{L}$, one can simply work backwards to determine the required sensitivity and allowable noise in a sensor. Inputting the data from Table 1.1 
into equations 2 and 3, the sensitivity for an electrode must be greater than 500 ADC counts per $\mathrm{nL} / \mathrm{L}$ to achieve the desired lower detection limit of $0.05 \mu \mathrm{L} / \mathrm{L}$.

\subsection{Why Measure $\mathrm{CO}_{2}$ and $\mathrm{O}_{2}$ Respiration Products?}

The conducted research focused on the electrochemical sensing of ethylene gas. However, a complete perspective on the sensing requirements for postharvest respiration of fresh fruit would include $\mathrm{CO}_{2}$ and $\mathrm{O}_{2}$ measurements. The respiratory quotient (RQ) is defined as the ratio of the rate of $\mathrm{CO}_{2}$ production to $\mathrm{O}_{2}$ uptake and is an indicator of fruit health under cold storage ${ }^{22}$.

1.7 Why use Handheld Reflectance NIR Spectroscopy to Monitor Fruit Maturity? Mcglone found that the dry matter content of apples is directly linked to their storability ${ }^{23}$ and can be predicted by near infrared spectroscopy (NIR) ${ }^{24}$. Palmer linked dry matter in apples to improved consumer preference ${ }^{25}$. By applying these findings, a farm manager could develop a harvest criteria that includes dry matter, total soluble solids (approximation of sugar content or brix), firmness, total acid, and color based on nondestructive NIR measurements in the field ${ }^{26}$.

\subsection{Combining the Two Sensing Platforms to Optimize Fruit Quality} Portable electrochemical sensing platforms and handheld reflectance spectrometers could be used together by postharvest researchers and their industry counterparts to ensure that consumers experience the best possible eating quality and shelf life of fresh 
produce. Higher dry matter fruit could be sorted by spectrometers and then stored in controlled atmosphere storage for an extended period. Lower dry matter fruit could be sorted by spectrometers and either stored for a shorter amount of time or sent directly to the next step along the supply chain. An electrochemical sensor system for ethylene, $\mathrm{CO}_{2}$, and $\mathrm{O}_{2}$, could be used by the Packhouse Quality Assurance Manager to monitor the respiration of apples while in cold storage. The ethylene concentration, along with the respiratory quotient (RQ), could be monitored with the electrochemical sensor system. If the ratio of $\mathrm{CO}_{2}$ to $\mathrm{O}_{2}{ }^{27}$ is no longer within ideal range or if the ethylene gas ${ }^{28}$ begins to increase, the sensing system could signal an alarm to vent the room to readjust the controlled atmosphere. The spectroscopic and electrochemical sensing methods could ensure that the industry quality standards are maintained along the supply chain.

\subsection{Summary}

The improvements to sensors and portable instruments for postharvest agriculture conducted in this study would reduce food waste and improve the quality of fruit reaching the consumer by providing critical information to manage produce along the supply chain. The development of inexpensive, repeatable, and accurate measurement systems for ethylene gas can prevent food spoilage during storage and transportation ${ }^{29}$. The use of spectroscopy in the orchard has the potential to add value to the farmer's crop by only picking fruit at the ideal maturity. Before these benefits can be realized, 
methods to collect stable and accurate data such as those proposed in this research must be developed. This was the objective of this investigation.

\subsection{Statement of Novelty}

This research has led to the following contributions in the area of fruit monitoring.

1. To the best of author's knowledge the use of ethephon as a calibration source for an electrochemical gas sensor has not been previously reported. A related project by Park $^{30}$, discusses the fabrication and use of ethephon tablets in a buffered solution as an alternate means of generating ethylene gas for induced ripening applications.

2. The use of a filter prior to the sensor to remove interfering compounds in and of itself is not novel. However, experimental data under simulated storage conditions of apples have not been previously reported.

3. The research for points 1 and 2 is presented in Chapter 2 and was published in Sensors and Actuators $B^{31}$.

4. The investigation of the use of white reference corrections for handheld spectrometers to account for environmental conditions has not been previously reported. Although similar work for inline sorting machines has been reported ${ }^{32,33}$, further work was warranted as the packhouse allows for more control of environmental conditions. 
5. PLSR was developed in $1964^{34}$, and with the development of the PC soon came to dominate NIR chemometrics. During this same time period Karl Norris ${ }^{35,36}$ development the derivative quotient method or DQM. To the best of author's knowledge, there are no prior, which contrast of calibration transfer with DQM and PLSR.

\subsection{Outline of Subsequent Chapters}

Chapter 2 focuses on the improving the performance of a commercial electrochemical ethylene sensor via controlled ethylene generation in situ and the use of an inline filter to improve sensor accuracy. This chapter was published in Sensors and Actuators $B^{31}$.

Chapter 3 provides additional background on the use of a handheld diode array spectrometer for maturity estimations in thin-skinned fruit. An experiment that optimizes the distance between the sample fruit and detector is also presented. A portion of chapter 3 was pervious published in NIRNews ${ }^{37}$.

Chapter 4 explores the role of environmental conditions on the ability of handheld spectrometers to collect reproducible spectra. Methods to increase the performance of hand held spectrometer performance under field conditions are presented. A portion of chapter 4 was pervious published In NIRNews ${ }^{38,} 39$.

Chapter 5 presents the first comparison of the derivative quotient method, DQM, and partial least squares regression, PLSR, for calibration transfer. The portions of the chapter will be submitted for publication to the Journal of Near Infrared Spectroscopy. 


\section{Chapter 2}

Optimizing the Performance of a Commercial Electrochemical Ethylene Sensor via Controlled Ethylene Generation in Situ 


\subsection{Introduction}

Ethylene, which regulates fruit ripening processes in some species, is a molecule of central interest to postharvest researchers and technologists. There is a need for a portable, inexpensive, accurate, and repeatable sensor for infield or storeroom measurements. This chapter presents a post-harvest study of ethylene production of apples and inline polar hydrocarbon trap to remove interfering gaseous species. Additionally, the development of a field calibration protocol for a portable electrochemical gas sensor is presented. Complementary gas chromatographic (GC) assays provided an independent validation of these results.

To achieve meaningful measurements in field conditions an electrochemical (EC) sensor needs a periodic span and zero correction. 'Macview' (EMS, Netherlands) a commercial postharvest storage room monitoring system, utilizes an onboard ethylene gas cylinder for a calibration standard to provide a periodic span correction. Whereas, the F-900 (Felix Instruments, USA) requires an external calibration cylinder for periodic span corrections. Here I present an economical alternative to a calibration gas cylinder for in field periodic span correction. It exploits the kinetically controlled reaction of ethephon in a buffer solution to liberate a headspace with a stable concentration of ethylene gas. Ethephon and the buffer solution are inexpensive compared to the costs of using a cylinder of calibration gas standard. Ethephon decomposes in aqueous solutions at $\mathrm{pH}$ values $>3.5$, releasing ethylene, and furthermore, above $\mathrm{pH}$ values $>4.5$ the decomposition is quantitatively predictable making it feasible to use as a headspace calibration ${ }^{40}$. Based on 
this chemistry, Park et al. ${ }^{30}$ used an ethephon containing tablet to react with a $\mathrm{KOH}$ solution at $\mathrm{pH}>7$ and liberate $\mathrm{a} \sim 2 \mu \mathrm{L} / \mathrm{L}$ headspace concentration of ethylene.

The ethephon decomposition rate is determined by multiple variables including the ethephon concentration, temperature, and $\mathrm{pH}$, with a more alkaline $\mathrm{pH}$ resulting in more efficient decomposition ${ }^{41,42}$. The chemical decomposition of ethephon to ethylene follows Equation $4^{43}$.

$\mathrm{Cl}-\mathrm{CH}_{2}-\mathrm{CH}_{2}-\mathrm{PO}_{3} \mathrm{H}_{2}+\mathrm{OH}^{-} \rightarrow \mathrm{Cl}^{-}+\mathrm{CH}_{2}=\mathrm{CH}_{2}+\mathrm{H}_{3} \mathrm{PO}_{4}$

Thus, ethephon decomposition releases phosphoric acid and a chloride anion in addition to ethylene. $\mathrm{H}_{3} \mathrm{PO}_{4}$ reduces the $\mathrm{pH}$ of the reaction mixture to suppress the release of ethylene ${ }^{44,45}$. Therefore, in the present method, the reaction is carried out in a buffered solution. To the best of our knowledge, the controlled release of ethylene from buffered solution has not been used for ethylene sensor calibration.

Cross-sensitivity occurs when more than one species of gaseous molecule reacts at the working electrode's surface ${ }^{18,19}$. The extra electrons released by the oxidation of nontarget molecules are counted by the electrical circuit and lead to erroneously high estimations of the ethylene concentration, which compromises the sensor accuracy. This lack of selectivity results in cross-sensitivity. Gas chromatography solves the problem of cross-sensitivity by use of a column to separate gaseous species before the sensor 
detection. A potential solution to the issue of cross-sensitivity for EC sensors is the use of an aqueous trap before the sensor so that the fruit headspace matrix (containing unsaturated polar VOCs) is reduced mostly to ethylene, which has low solubility in water $\left(120 \mathrm{ppm} \text { at } 20^{\circ} \mathrm{C}\right)^{46}$. Common cross contaminants include alcohols, esters, aldehydes and to a lesser extent terpenes ${ }^{47,48}$.

The concept of gas filtration before its entry into an EC sensor is not new. One existing pre-sensor trap utilizes a polymer with specific functional groups to capture interfering compounds, which are permanently bound or reduced to non-interfering compounds. However, this design can allow ethylene oxide, a polar compound to pass freely to the sensor while absorbing and oxidizing other polar compounds such as alcohols ${ }^{49}$. This method as used by Warbuton and Richard (2009) could be adapted to allow ethylene to freely pass while trapping polar compounds. However, the cost of a custom synthesized polymer, along with its periodic replacement, might be a limiting factor. Another technology uses a silver doped alumina cold finger to selectively adsorb ethylene. Later, the cold finger is heated to $115^{\circ} \mathrm{C}$ by a $50 \mathrm{~W}$ lamp to release the captured ethylene. The cold finger can also be used to concentrate low levels of ethylene, and then release it to the sensor ${ }^{50}$. The necessary heating and cooling steps for a cold finger are not practical in the field.

Primary goals of this study are twofold: (1) to improve the accuracy of an EC sensor by integrating an aqueous trap to filter ethylene from interfering polar organic compounds, (2) to provide a field-usable, integrated compact chemically generated ethylene 
calibration standard that eliminates the need for a cylinder of calibration gas. The present study utilizes an EC sensor (Membrapore, Switzerland). The sensor current is correlated to the ethylene concentration. Any other unsaturated or easy to oxidize species in the instrument head-space can increase the error in the measured ethylene concentration. A simple aqueous trap removes polar organic compounds. However, during continuous operation of the sensor under steady flow conditions, the trap becomes saturated allowing excess non-target material to reach the sensor, resulting in an erroneous estimation of ethylene concentration. The polar compounds have a much higher solubility in water, and hence the trap provides a longer time window over which these compounds are removed before saturation. Subsequently, the trap would need regeneration/replacement as discussed later. Note, ethylene, with its low solubility in water, would be expected to take shortest time (t1) for water saturation while alcohols are expected to have the longest $\left(t_{2}\right)$. An accurate value of ethylene would require measurement of sensor response at time $t$ such that $t_{1}<t<t_{2}$, preferably just after $t_{1}$. A pulse mode of operation, i.e., alternating electrochemical detection and trap regeneration cycles allow removal of polar organics from the trap and thus provides a better accuracy of measured ethylene content while eliminating the need to replace trap water. Optimization of the detection and regeneration cycles is reported. 


\subsubsection{Materials and methods}

\subsubsection{Preparation of Ethephon $\mathrm{KCl}$ Powder}

To achieve an ethylene concentration in headspace below $1.0 \mu \mathrm{L} / \mathrm{L}$ requires between $1-10$ mg of ethephon (CAS Number: 16672-87-0 obtained and used as received from Sigma Aldrich) per run. As discussed in supplementary material ethephon was mixed with $\mathrm{KCl}$ (Sigma- Aldrich) to produce ready to use solid pellets containing $3.5 \%$ ethephon.

\subsubsection{GC Measurements}

Compositional analysis employed a syringe (HAM81020, USA) to collect $100 \mu \mathrm{L}$ of gas from the exit port (Figure S-1 in Appendix A) of a F-900 (Felix Instruments, USA) portable gas analyzer which was manually injected into a HP6580 series II GC-FID with a custom packed column HS-A 80/100 3.3 m length. 2.0 mm ID, 3.175 mm OD (Restek, USA). The GC-FID oven parameters were as follows: $\mathrm{H}_{2}$ flow at 110 psi, $\mathrm{O}_{2}$ flow at $47 \mathrm{psi}, \mathrm{N}_{2}$ flow at 45 psi, inlet temperature $230{ }^{\circ} \mathrm{C}$, detector temperature $220^{\circ} \mathrm{C}$, oven temperature $150{ }^{\circ} \mathrm{C}$. Polar compounds emitted by the fruit dissociatively adsorb on the column matrix. Therefore the GC method was unable to quantify their concentrations. Compositional analysis of polar compounds before and after the polar trap was attempted with an HP6980 GC-MS, using an HP-5ms column with a length of $30 \mathrm{~m}$. The GC-MS oven parameters were as follows: inlet temperature $270^{\circ} \mathrm{C}$, interface temperature $240{ }^{\circ} \mathrm{C}$, oven temperature $60^{\circ} \mathrm{C}$. 


\subsubsection{Startup procedure of F-900 for Ethephon Headspace}

At the outset, a continuous measurement mode of the F-900 was initiated with an external closed gas circulating loop by connecting the inlet and outlet ports with Tygon tubing ${ }^{51}$. The flow rate of $\sim 75 \mathrm{~mL} / \mathrm{min}$ was controlled by the onboard pump inside the F900. Then the automated zero-set routine was run on the instrument. The purpose of the automated zero is to account for the daily fluctuations in average background signal from the EC sensor. Following the zero-set routine, the external closed loop was removed, and a cylinder of $1 \mu \mathrm{L} / \mathrm{L} \pm 5 \%$ ethylene from Airgas was connected to the input port of the F900. The built-in instrument calibration routine was then initiated. The calibration for the EC sensor (Membrapore, Switzerland) used in the F-900 was stable and read within $\pm 10 \%$ over the three-week data collection period.

\subsubsection{Preparation of Calibration Standards using Ethephon}

Three separate ethephon/KCl mixtures $(50,200,400 \mathrm{mg})$ dissolved in phosphate buffer solutions provided known concentrations of liberated ethylene. The corresponding concentration of ethephon in buffer solutions were $0.375,1.5$ and $3.0 \mathrm{mM}$, respectively. The phosphate buffer $(6.9 \mathrm{pH})$ consisted of $0.2470 \mathrm{~g}$ dibasic sodium phosphate and 0.0900 $\mathrm{g}$ of monobasic sodium phosphate dissolved in $32 \mathrm{~mL}$ of DI water. During the calibration, the reaction mixture occupied the input conditioning chamber of F-900 (See Figure 2.1). The duration of calibration measurements were about 5-10 minutes.

\subsubsection{Measurement of Ethylene Released by Ethephon Reaction}


An additional external conditioning chamber containing potassium permanganate pellets (Purafil, USA) was connected to the input port of the F-900 (shown schematically in Figure 2.1). The purpose of this chamber was to oxidize residual hydrocarbons in the air to ensure that the electrochemical sensor detected only ethylene released by the buffering reaction. Neither the F-900 nor GC detected a measurable concentration of hydrocarbons or polar VOCs in the purified ambient air. For the sensor calibration, the purified ambient air, bubbled through the input conditioning chamber on the back of the F-900, transported ethylene from the buffered ethephon reaction solution. The passage of air through this chamber resulted in a known concentration of ethylene depending on the ethephon reaction conditions at the fixed airflow rate ( $75 \mathrm{~mL} /$ minute) employed by F-900. The F900 operated in a continuous mode for data collection. Calibrated signal responses from the F-900 allowed detection of $n L / L$ concentrations of ethylene via its EC oxidation. Finally, the air exited the F-900 to be manually sampled for analysis by the GC. Note, not all the ethylene entering the EC sensor compartment was oxidized completely but that the signal reported by the F-900 was proportional to the concentration of ethylene in the input gas stream. The gas exiting the sensor still contained unreacted ethylene which was manually sampled for GC analysis. Air temperature as reported by the F-900 during data collection was $26^{\circ} \mathrm{C}$. 


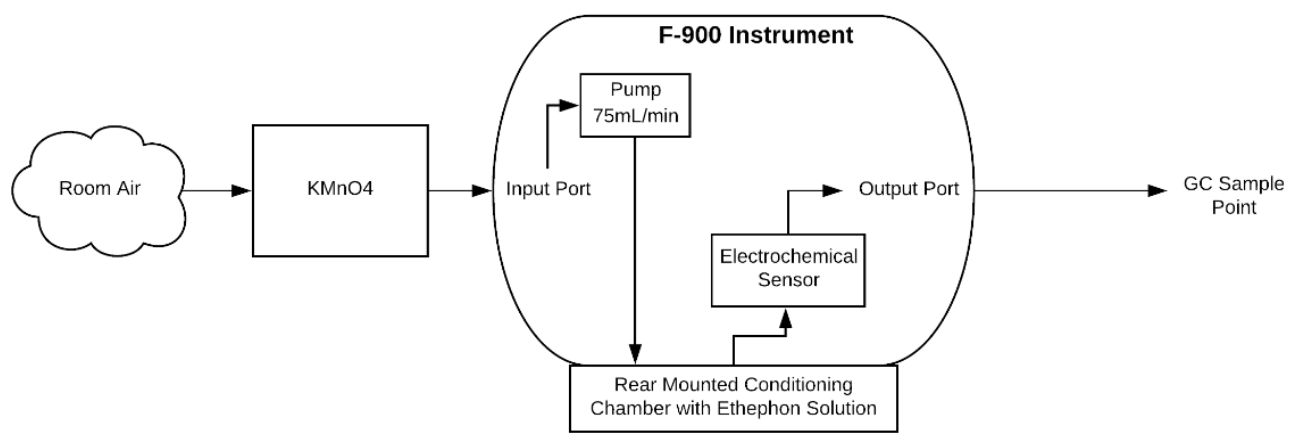

Figure 2.1. Block diagram of the external setup for ethephon reaction. For the internal operation of the F-900 and the physical location of the built-in input and output conditioning chambers see Figure S-1, Appendix A.

\subsubsection{Measurements of Ethylene Production from Apple}

Before data collection, $45 \mathrm{~mL}$ of deionized water (DiW) replaced the Ethephon reaction mixture in the input conditioning chamber of F-900. The DiW provided a trap for polar VOCs (Figure S-2, Appendix A). The output conditioning chamber contained potassium permanganate pellets. The preprogrammed set-zero routine in the F-900 (firmware V4.7.0.4) did not account for any background signal from the DiW from the input conditioning chamber. The external closed loop mode of F-900 removed volatile compounds dissolved in DiW (Figure S-3, Appendix A) in the conditioning chamber by outgassing. Data logging raw ADC counts from the sensor established when the stable background signal was realized. Typically, it took a few minutes to stabilize the background signal for the counts. The stable ADC count value was then manually entered to set new zero in the calibration menu of the F-900. As mentioned above, F-900 operated in a 
continuous measurement mode (by disconnecting the external closed loop), with both the input and output conditioning chambers enabled in software.

Polar trap efficiency measurements from a headspace from seven 'Gala' apples, with a mass of $1.65 \mathrm{Kg}$, loaded into a $2 \mathrm{~L}$ chamber (Felix Instruments). Typical equilibrium time was about one hour to reach a steady-state ethylene production rate (at $25.8 \pm 0.3^{\circ} \mathrm{C}$ ) under of $100 \mathrm{~mL} / \mathrm{min}$ constant flow rate of purified ambient air supplied by an external pump (Figure 2.2, (bottom). The uninterrupted headspace withdrawal was necessary to ensure steady-state conditions around the apples was not disturbed by the operating mode of the F-900. A T junction placed between fruit chamber and F-900 acted as a vent and also allowed GC sampling of the headspace before entering the sensor. The F-900 periodically subsampled the apple headspace (analyte airstream) by withdrawing it at a rate of $75 \mathrm{~mL} /$ minute.

Measurement of apple headspace used a "special" F-900 measurement mode combining sampling and trap regeneration periods. The measurement period of 6 min allowed sufficient time for the EC sensor in the F-900 to come to equilibrium with the analyte airstream. Similarly, the regeneration time for the closed loop regeneration period was 10 minutes. The internal operation of the F-900 in the measurement and regeneration period of the special mode is displayed in Figure S-2 and Figure S-3 of the supplemental material. 


\subsubsection{Validation of Reduction of Volatile Organic Compounds in Airstream}

An additional VOC headspace experiment was conducted with 16 'Lady Alice' apples, stored in a $5 \mathrm{~L}$ fruit container, under otherwise identical experiential conditions. The GCFID used for confirmation of ethylene concentration was replaced with a GC-MS for analysis of polar compounds. The GC sample points before and after the aqueous trap, shown in Figure 2.2, were bubbled in $1 \mathrm{~mL}$ samples of methanol to captured and concentrated organic compounds present in the airstream. GC-MS injections employed an aliquot of $100 \mu \mathrm{L}$ of the bubbled methanol. The GC data (not shown) found a 38\% reduction in a polar VOC peak before and after the polar trap. The MS fragments were able to identify a few compounds trapped such as C3-C7 alcohols as well as terpene related compounds as shown in the supplementary material (Figure S-5 through S-7 and Tables S-1, S-2 in Appendix A). 

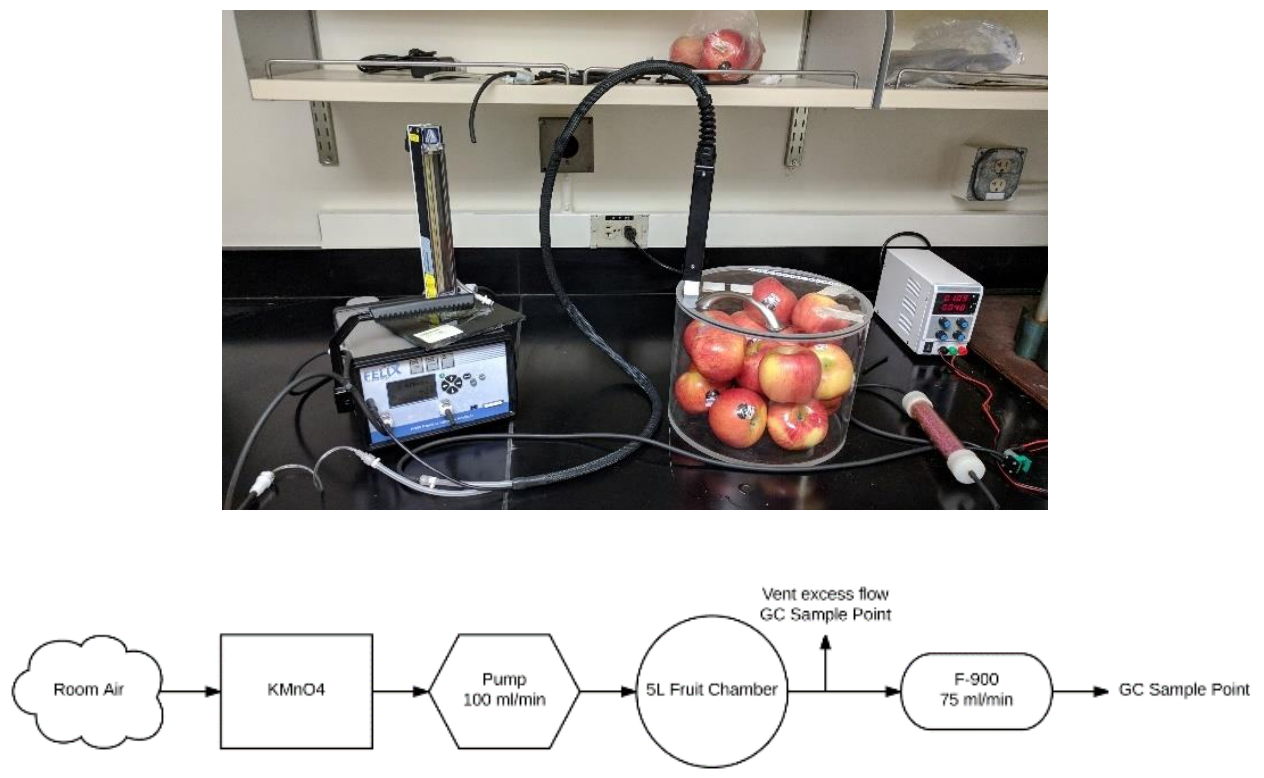

Figure 2.2. Top) Experimental setup. Bottom) Schematic for ethylene production study. For the internal operation of the F-900 and the location of the built-in input and output conditioning chambers see Figure S-1.

\subsubsection{Results and Discussions}

\subsubsection{Ethephon Calibration Standard}

Ethanol dehydration can be used to industrially produce ethylene. However, further purification is needed for use as a calibration source ${ }^{52}$. Zhang reported a method to fully decompose ethephon into a $2 \mathrm{~L}$ chamber. A GC study indicated that the $2 \mathrm{~L}$ chamber contained an expected concentration of ethylene based on the stoichiometric conversion of ethephon to ethylene ${ }^{44}$. Given sufficient time under highly basic conditions, the method of complete conversion from ethephon to ethylene could replace cylinders of calibration gas. 
As reported by Klein ${ }^{42}$ ethephon, under the conditions of this experiment $\left(\mathrm{T}=25^{\circ} \mathrm{C}\right.$ and $\mathrm{pH}=6.9)$, decomposes with a first order rate constant $(\mathrm{k})$ of approximately $10^{-5} \mathrm{sec}^{-1}$. The rate constant implies $19.5 \mathrm{hr}$ half-life for the reaction. As measurement periods are only 5-10 minutes long, the ethephon decomposition is effectively a zero-order reaction. For a pseudo-first-order reaction (Note in eq. $1\left[\mathrm{OH}^{-}\right]>>\left[\right.$Ethephon]) the concentration of $\mathrm{C}_{2} \mathrm{H}_{4}$ is equal to the concentration of ethephon degraded. It varies as $C(t)=C_{0}\left(1-e^{-k t}\right) \approx C_{0}(1-(1-$ $k t))=C_{0} k t$, when $k t<1$, where $C_{0}$ is the initial concentration of ethephon. Thus for measurement duration of $\mathrm{t}=300 \mathrm{~s}$ results in zero-order reaction kinetics, i.e., $\mathrm{kt}=3 \times 10^{-2}$ $\ll 1$. Under experimental conditions, the rate of production of $\mathrm{C}_{2} \mathrm{H}_{4}$ equals $\mathrm{C}_{0} \mathrm{k}$, which is a time-independent constant proportional to the initial concentration of ethephon. Therefore, at a constant rate of inflow to F-900, the fixed concentration of ethylene in the carrier gas provides a suitable calibration standard for infield sensor calibration.

For this study, it was desirable to generate an on-demand supply of calibration gas using the input conditioning chamber located on the back of the F-900. The reaction of ethephon to ethylene generates a stable concentration of ethylene gas over the 5 minute period required for a span calibration of the F- $900^{41}$. The F-900 requires approximately $400 \mathrm{~mL}$ of gas over the $5 \mathrm{~min}$ period. By adjusting the quantity of $\mathrm{KCl} /$ ethephon added to the buffer solution, a span point can be adjusted to ensure the accuracy of the calibration at the expected concentration for an experiment.

The response of the EC sensor (Figure 2.3) to the ethephon generated ethylene is visually equivalent to the standard calibration gas. Figure 2.3 depicts a solid line for the 
electrochemical sensor's response when exposed to $1 \mu \mathrm{L} / \mathrm{L} \pm 5 \%$ ethylene calibration gas standard (Airgas). The dashed line shows the response of the sensor to $\sim 1 \mu \mathrm{L} / \mathrm{L}$ of ethylene generated by a $250 \mathrm{mg}$ ethephon/KCl pellet dissolved in $32 \mathrm{~mL}$ of buffer solution. Except for a slightly longer rise time for the latter, both the systems saturate at $1 \mu \mathrm{L} / \mathrm{L}$ within three minutes. Furthermore, the standard deviations of the recorded ADC counts between 5 and 6 minutes are comparable $\left(\sigma=0.01 n L L^{-1}, \mu=0.87 n L L^{-1}\right)$ for both the sources of ethylene. The GC calibration curve of ethephon generated ethylene appears in the supplementary information (Figure S-4, Appendix A).

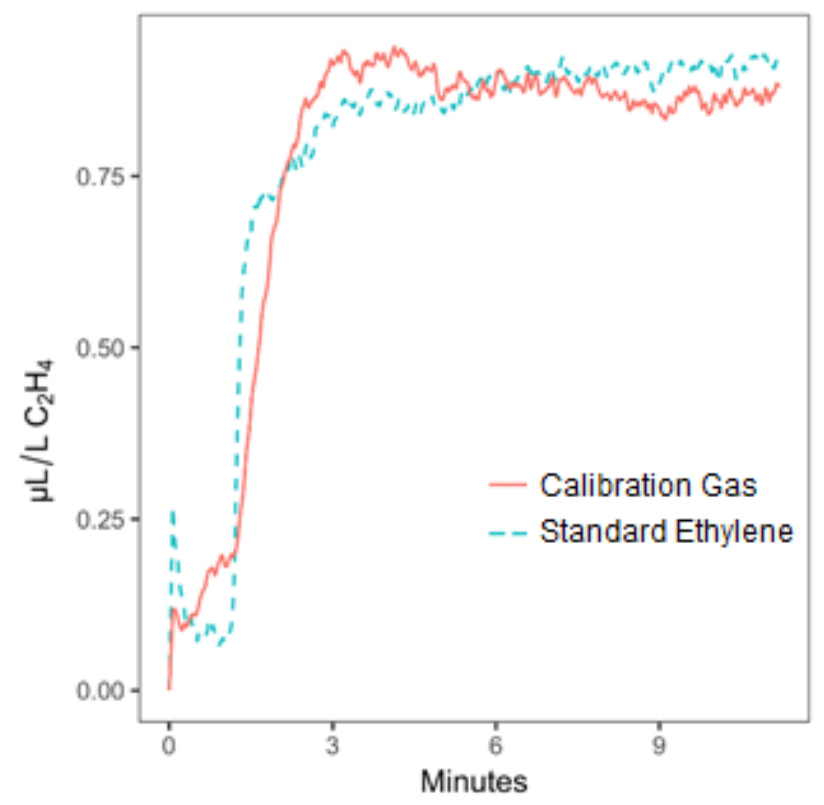

Figure 2.3. Electrochemical response as recorded by the F-900 for a standard calibration gas and ethephon generated ethylene. 
Table 2.1. Dependence of ethylene concentration on ethephon concentration as measured by the electrochemical and the GC-FID methods.

\begin{tabular}{lll}
\hline $\begin{array}{l}\text { Ethephon } \\
\text { concentration }(\mathrm{mM})\end{array}$ & $\begin{array}{l}\mathrm{GC} \text { Reported } \\
\text { Concentration }(\mu \mathrm{L} / \mathrm{L})\end{array}$ & $\begin{array}{l}\text { F-900 Reported } \\
\text { Concentration } \\
(\mu \mathrm{L} / \mathrm{L})\end{array}$ \\
\hline 0.375 & $0.28 \pm 0.03$ & $0.29 \pm 0.03$ \\
1.5 & $0.49 \pm 0.03$ & $0.59 \pm 0.06$ \\
3.0 & $1.50 \pm 0.03$ & $1.8 \pm 0.2$ \\
\hline
\end{tabular}

Table 2.1 compares GC measured ethylene concentration for the samples collected from the outlet of the F-900. The F-900 and the GC results are in reasonable agreement with the concentration of ethylene liberated from varying concentrations of ethephon $/ \mathrm{KCl}$ mixture in a buffered solution. Higher uncertainty in the electrochemical result at the highest concentration of ethylene is due to reaching the upper limit for $\mathrm{C}_{2} \mathrm{H}_{4}$ sensing on F900.

\subsubsection{Operational principle for polar volatile organic compounds trap}

All fruits emit a complex headspace of volatile organic compounds (VOC) ${ }^{53}$. Apples emit mainly esters, alcohols, aldehydes, ketones, and acids ${ }^{14,21}$. An inline polar volatile organic compound trap, as shown in Figure S-2, improves the selectivity of the EC sensor for ethylene. The trap allows ethylene and nonpolar volatile compounds to pass freely to the sensor while trapping a majority of the polar components of the headspace. The trap operates on the chemical principle that 'like dissolves like' and that the direction of absorptivity follows a concentration gradient from high to low. Bubbling the air stream 
through $45 \mathrm{~mL}$ of DiW water removes polar VOCs thus preventing them from reaching the EC sensor. Other high vapor pressure polar liquids could be substituted for the water. However, they must not produce an interfering signal. In principle, micellar surfactant solutions can trap larger nonpolar hydrocarbons such as terpenes; but foaming in the chamber prevented the use of such alternate trapping liquids. Replacing common surfactants with nonfoaming surfactants might provide a better solution.

\subsubsection{Theory of regenerating the trap}

By running the polar hydrocarbon trap in a closed loop as shown in Figure S-3 with the EC sensor and potassium permanganate in the loop, reduces the concentration of polar VOCs in the air stream. Once the concentration of polar VOCs in the air stream is less than the solubility limit of concentration in the trap, the trap begins to emit polar VOCs to the airstream. This protocol allows the polar trap to regenerate its capacity. GC elution data collected from the vent port and output of F-900 (Figure 2.4) show that there is a negligible loss of ethylene upon insertion of the polar trap. 


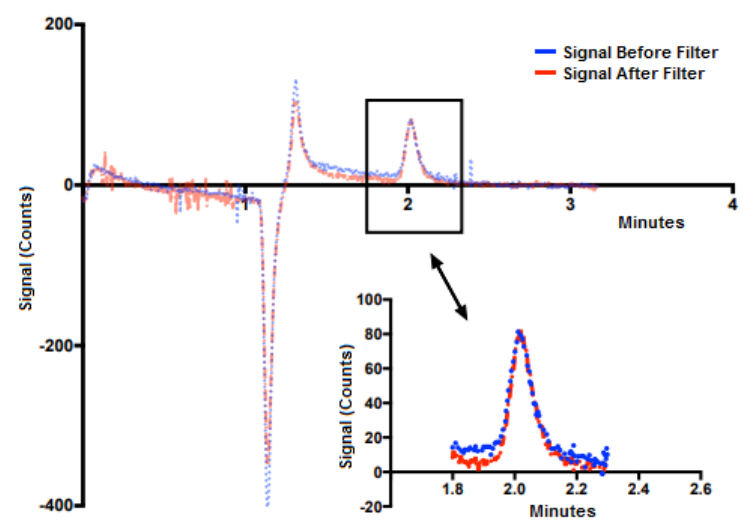

Figure 2.4. Chromatograms of airstream collected before and after polar VOC trap for the apple headspace. Any ethylene loss to trap is within the margin of error of the GC. The reported GC concentration for both before and after the filter is $1.00 \pm 0.03 \mu \mathrm{L} / \mathrm{L}$.

The response of the EC sensor, while operating in a continuous detection mode, to ethylene from the apple headspace without the use of the aqueous VOC trap is shown in Figure 2.5. The sensor output rose rapidly to about $1 \mu \mathrm{L} / \mathrm{L}$ in the first six minutes, which was similar to the $1.00 \pm 0.03 \mu \mathrm{L} / \mathrm{L}$ concentration detected by GC. Subsequently, the sensor response followed a slow increase to another plateau at $1.5 \mu \mathrm{L} / \mathrm{L}$ after about 15 minutes. A GC sample collected while the EC sensors recorded an ethylene headspace concentration of $1.5 \mu \mathrm{L} / \mathrm{L}$ confirmed that the actual ethylene concentration remained steady at $1.00 \pm 0.03 \mu \mathrm{L} / \mathrm{L}$. This finding suggests the need to refresh the aqueous trap after 6 minutes of sensor operation as explained furhter below. 


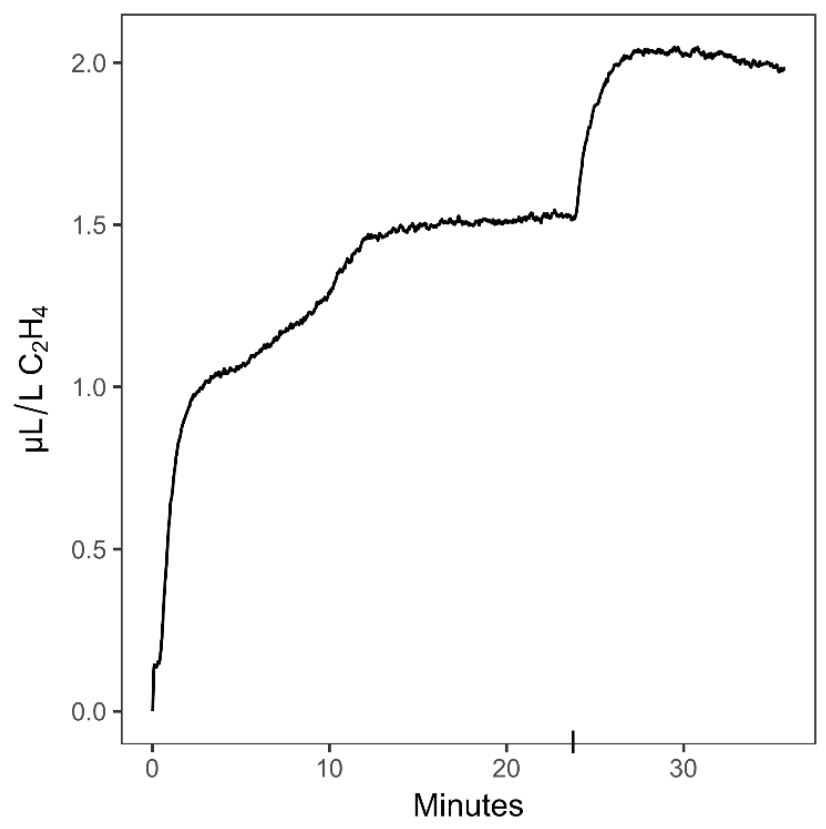

Figure 2.5. Continuous measurement of apple headspace; the water trap is enabled for the first 25 minutes without closed loop regeneration. For the last 11 minutes (25-36 minutes) the sensor is exposed to unfiltered apple headspace.

The second desorption isotherm beginning at $\sim 7$ minute (Figure 2.5 ) is likely the result of saturation of water in the polar trap. As the polar trap approaches saturation, it would allow leakage of non-ethylenic polar VOCs. The isotherm for the second stage of desorption flattens off around the 12-minute mark and reaches an equilibrium at 15 minute with a reported ethylene concentration of $\sim 1.5 \mu \mathrm{L} / \mathrm{L}$. The extra $0.5 \mu \mathrm{L} / \mathrm{L}$ ethylene detected would correspond to polar VOCs that are no longer removed by the trap. In Figure 2.5 , the water trap is disabled at 25-minute mark and the sensor is exposed directly to total amount of VOCs in the headspace. The sensor records an effective $\mathrm{C}_{2} \mathrm{H}_{4}$ concentration of $\sim 2 \mu \mathrm{L} / \mathrm{L}$, when exposed to the unfiltered headspace. These data imply that the polar trap although saturated with respect to a certain class of polar VOCs, it still 
effective in blocking other more soluble VOCs from entering sensor chamber. The actual ethylene concentration as determined by the $\mathrm{GC}$ is $1.00 \pm 0.03 \mu \mathrm{L} / \mathrm{L}$. This over-estimation by the sensor without the trap is due to the various cross-sensitivities of the total VOCs in the headspace, which contribute to a higher but false ethylene signal.

Comparing the signals from unfiltered (between 25-35 minutes in Figure 2.5) vs. filtered headspace (1-25 minutes) one can determine the efficacy of the aqueous trap in the system. The overall effect of the aqueous trap for these 'Gala' apple headspace, is the reduction of ethylene-like signal due to interfering species, which appears to contribute $\sim 50 \%$ of excess signal based on signal response curve. A regeneration period is necessary to remove the trapped polar compounds from the trap-water. Bubbling clean air through the trap during the regeneration period, removes the trapped polar compounds by their partitioning between air and water.

Figure 2.6 shows the response of the EC sensor to the apple headspace during several pulsed operating cycles of the F-900. The "special" F-900 pulsed trap regeneration and detection mode setting provides repeatable apple headspace measurements showing an ethylene concentration of $\sim 1 \mu \mathrm{L} / \mathrm{L}$. A GC sample was collected during every measurement period, and confirmed that the ethylene concentration was $1.00 \pm 0.03 \mu \mathrm{L} / \mathrm{L}$. As shown by the first 6 peaks (0-100 minutes), with the output chamber containing $\mathrm{KMnO}_{4}$ present in the closed loop regeneration, a duration of $10 \mathrm{~min}$ is sufficient to release and oxidize "all ( 85\%)" of the polar compounds from the water and recover the efficacy of the trap. It is important to highlight that despite the inclusion of $\mathrm{KMnO}_{4}$, the EC response baseline does 
not go back fully to zero, but returns to $\sim 0.15 \mu \mathrm{L} / \mathrm{L}$ after each regeneration cycle, thereby implying an incomplete (T90) removal of VOCs from the trap after 10 minutes. Removal of the $\mathrm{KMnO}_{4}$ containing output chamber from the closed loop regeneration, as demonstrated by the last two peaks (appearing between 100-139 minutes), results in a baseline movement and effectively higher $\mathrm{C}_{2} \mathrm{H}_{4}$ concentrations being recorded. From these studies, I conclude that a 10 minutes regeneration period for the aqueous trap is optimal for this application, on apple headspace. Depending on the actual headspace concentrations, one can further optimize the regeneration cycles by requiring that signal level to drop by $90 \%$ of the initial value. Similarly, a sensor detection period of 6 minutes can be optimized by performing continuous monitoring of the sensor output as indicated in Figure 2.4. The optimal detection period is established when the sensor response curve reaches the first plateau. In the case of apples, the first plateau is defined as the final 2.8 minutes of the measurement period. The standard deviation of ethylene readings over the first plateau is $0.01 \mu \mathrm{L} / \mathrm{L}$, which is equivalent to the standard deviation of readings of an ethylene standard. 


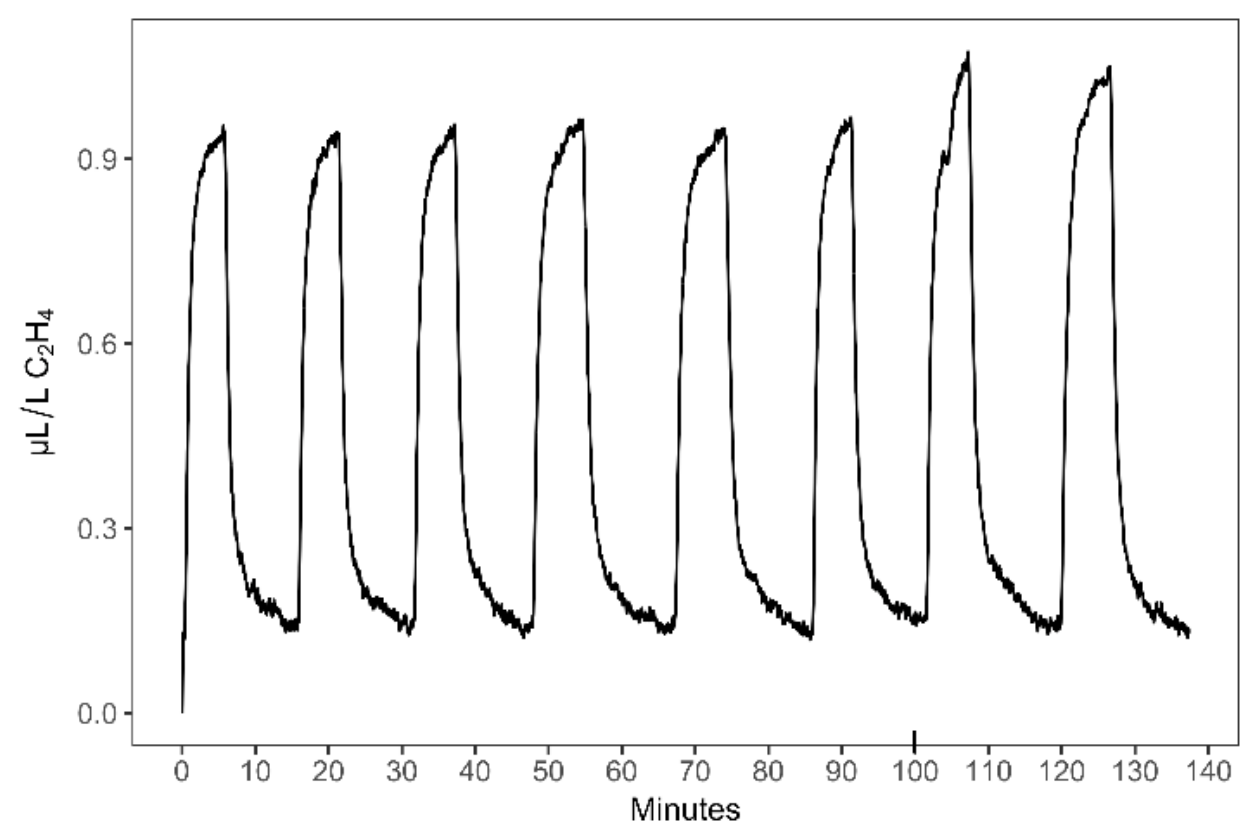

Figure 2.6. Six repeated measurements and regeneration cycles (0-100 minutes) of apple headspace with $\mathrm{KMnO} 4$ included in the closed loop regeneration of the trap taken in a "special" mode in the F-900. The last two peaks (100-140 minutes) are repeated measurements in a special mode without $\mathrm{KMnO} 4$ in the closed loop regeneration of the trap.

The regeneration period is likely to be a commodity (fruit type) or application-specific setting, as samples with higher total VOCs will very likely require more time to outgas and oxidize the captured compounds. When the EC sensor is exposed to the apple headspace without the trap, the F-900 calculates an ethylene reading of $\sim 2 \mu \mathrm{L} / \mathrm{L}$, whereas the actual ethylene concentration as determined by the $\mathrm{GC}$ is $1.00 \pm 0.03 \mu \mathrm{L} / \mathrm{L}$ (Figure 2.4). The special mode setting on the F-900 removes the contribution of interfering compounds from the analyte airstream, bringing the readings from the EC sensor of $1.90 \pm 0.20 \mu \mathrm{L} / \mathrm{L}$ into an agreement with the GC readings of $1.00 \pm 0.03 \mu \mathrm{L} / \mathrm{L}$. 
The ethylene production rate for the 7 'Gala' apples used in this experiment was found to be approximately $3.6 \mu \mathrm{L} / \mathrm{kg} \cdot \mathrm{hr}$. This production rate is comparable to the work of Watkins et al. ${ }^{54}$ who reported 'Gala' apple have an ethylene production rate between $0-4 \mu \mathrm{L} / \mathrm{kg} \cdot \mathrm{hr}$. at $20^{\circ} \mathrm{C}$. The Postharvest Department at UC Davis reports that 'Gala' apples are optimally stored at $0{ }^{\circ} \mathrm{C}$ under 90 to $95 \%$ relative humidity, under these conditions, 'Gala' apples produce ethylene gas at a rate of 4 to $12 \mu \mathrm{L} / \mathrm{kg} \cdot \mathrm{hr}^{6}$. The headspace, measurements given above were converted to an ethylene production rate using equation S-1, Appendix A.

\subsection{Conclusion}

This study describes a method to utilize ethephon to check the accuracy of an electrochemical ethylene calibration, essentially acting as a span check of the calibration. Furthermore, an interfering gas trap which does not impede the flow of ethylene can be used to improve the measurement selectivity of an electrochemical sensor to determine ethylene concentration.

Major portions of this chapter were previously published under:

Lerud, R. M., Beseau, D., Hale, C. M., Noll, C., \& Rananavare, S. B. (2019). Optimizing the performance of a commercial electrochemical ethylene sensor via controlled ethylene generation in situ. Sensors and Actuators B: Chemical, 281, 535-541.

doi:10.1016/j.snb.2018.09.127 


\section{Chapter 3}

Handheld diode array spectrometer for maturity estimations in thin-skinned fruit 


\subsection{Background}

Prediction of internal properties of thin-skinned fruit by use of shortwave NIR spectroscopy requires two primary assumptions. The first assumption is that the curvature of the fruit surface can be approximated as a flat surface regardless of spot size, so that the Beer-Lambert law, shown in equation 5, can be directly applied to model the changes in spectra.

$A=\alpha C L$

where the parameter $\mathrm{A}$ is absorbance, $\alpha$ is the absorptivity, $\mathrm{C}$ is the concentration of the analyte of interest, and $L$ is the path length that the light travels through the sample.

This approximation is made regardless of the spot size of the incident light. By approximating the fruit as a flat surface, the need for a geometric correction to account for fruit shape is avoided. This assertion is supported by the experimental work of Guthrie ${ }^{55}$ on the NIR spectroscopy of pineapple. Guthrie found that despite the thick skin, spatial compositional variation, and complex chemistry, NIR reflectance spectroscopy can be used to accurately predicted sugar content of pineapple.

The second assumption is that variation in the spectra between samples is linear and directly proportional to linear changes in the analyte of interest. In the case of spectroscopy of fruit, the analyte of interest is a physiological trait, such as sugar content. This assumption implies that any other physical or chemical variation between 
two pieces of fruit, which may alter the spectra, can be ignored. The second assumption can only be valid when variation between fruit is small, such as apples to apples. Any nonlinear effects in the spectra, such as scattering ${ }^{56}$, can be compensated for by use of chemometric pre-treatments, which are forms of signal processing ${ }^{57}$. Standard Normal Variate $^{58}$ (SNV) and Multiplicative Scatter Correction ${ }^{59}$ (MSC) are two signal processing approaches commonly used to pretreat spectra for baseline differences due to path length or scattering effects (both multiplicative and additive) ${ }^{60,61}$. Additionally, first and second derivatives are often taken of the spectra prior to multivariate analysis. The work of Guthrie also found that pre-treatment of the spectra, prior to multivariate analysis, mitigated variation in surface reflections between samples ${ }^{55}$. If both assumptions are valid, then the Beer-Lambert law can be used to approximate the covariance between the changes in spectra and reference value. Further discussions on the source of nonlinear effects, and common misconceptions in multivariate analysis of NIR spectroscopy are presented by Difoggio ${ }^{62}$. An additional benefit of assuming that changes in spectra are only due to changes in the analyte of interest is that a single spectrum window such as $729-975$ nanometers can be used by the multivariate math (discussed in in section 3.2.1). This can explain changes for several analytes of interest, such as sugar content and dry matter content of an apple. The sugar content of fruit is measured in "degrees Brix" by means of a digital refractometer. $1^{\circ}$ Brix is equivalent to 1 $\mathrm{mg}$ of sugar in $100 \mathrm{~mL}$ of water. The term degrees of Brix is due to the linear relationship between Snell's law and sugar concentration, i.e., every ${ }^{\circ}$ Brix bends the light passing 
through a refractometer by an additional degree relative to angle of pure water.

Although ${ }^{\circ}$ Brix in theory is a measure of sugar content only, in reality it is a measure of all solutes in the liquid sample. In the case of fruit juice, the term Total Soluble Solids (TSS) can be used interchangeably with degrees Brix.

\subsubsection{Background on Multivariate Math}

A handful of common methods, including Machine Learning, Principle Component Regression, and Partial Least Squares Regression, have been shown to successfully find regression between the spectra and reference values ${ }^{63,64}$. A detailed review of the theory and use of shortwave NIR for fruits and vegetables has been reported by B.M. Nicoli65. The work presented in this thesis will primarily focus on Partial Least Squares Regression, PLSR, as it is an industry-standard chemometric method ${ }^{66-68}$. A useful primer on the theory of Partial Least Squares Regression in advanced linear algebra has been published by Paul Geladi and Bruce Kowalski ${ }^{69}$. The use of PLSR for the multivariate design of experiments is discussed by Wold ${ }^{70}$. The PLSR analysis presented in this thesis was calculated in Unscrambler 10.3 software package..

Unlike traditional Least Squares, as shown in equation 6, Partial Least Squares regression does not suffer from the collinearity issues of finding $\left(X^{\prime} X\right)^{-1}$. PLSR overcomes this limitation by an iterative method that estimates $Y$ from the covariance of $X$ (dependent variables) and Y (independent variables). A simplified model of PLSR consists of independently decomposing $X$ and $Y$ into a linear combination of rank 1 matrices, 
shown in equation 7. Subsequently, regressing upon these rank 1 matrices results in a transformation vector also known as a regression or $\mathbf{B}$ vector. $\mathrm{Y}$ can be estimated by taking the inner product of $\mathrm{X}$ and $\mathrm{B}$. If $\mathrm{X}$ represents the spectra of an unknown sample, then the inner product will predict the $\mathrm{Y}$ or reference value of the unknown spectra, equation 8.

$B=\left(X^{\prime} X^{\prime}\right)^{-1} X^{\prime} y \quad[6]$

where $\mathbf{B}$ is a regression vector, $\mathbf{X}$ is a matrix containing spectra row-wise and $\mathbf{y}$ is $\mathbf{a}$ vector of corresponding reference values such as sugar content.

$X=T P^{\prime}+E$

where $\mathrm{X}$ is the independent variable, $\mathrm{T}$ is the scores matrix of $\mathrm{X}, \mathrm{P}$ is the loading matrix of $\mathrm{X}$, and $\mathrm{E}$ represents some residual effect.

$\hat{\mathrm{Y}}=B^{t} X$

where $\hat{Y}$ is an estimate of $Y, B$ is the regression vector, and $X$ is a matrix containing the independent variables.

Upon successful regression of nonlinear iterative partial least squares (NIPLAS) routine, the number of principle components (vectors) must be selected as a basis set.

Overfitting can occur when the principle components that provided no benefit to the regression are included in the basis set. The relative benefit of each successive principle component can be gauged from the root mean square error graph shown in Figure 3.1. There is a diminishing return in the change of the error between 8 and 9 principal components. As such, 9 principle components would be an over fit, so 8 components are selected. 


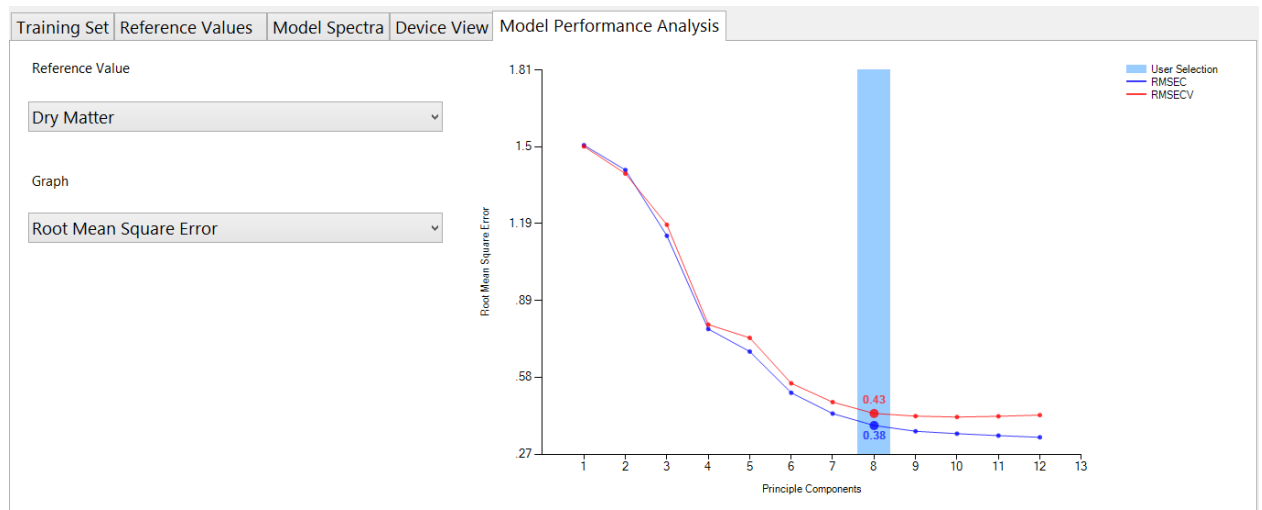

Figure 3.1. Determining the number of principle components selected is indicated by the blue bar in the root mean square error graph. The red line indicates the root mean square error of leave-one-out cross-validation. The blue line indicates the root mean square error of calibration. Apple dry matter PLSR calibration in the model builder software provided by Felix Instruments.

\subsubsection{The Importance of Reference Method Accuracy In PLSR}

As Near Infrared Spectroscopy (NIRS) moves from the lab to field-ready handheld spectrometer units, the NIRS community needs to develop simple examples and language suitable for farmers, QA technicians, and other operators. From personal experience the author has found people new to the use of NIRS in agriculture often ask, "how good of a model can I make?". The answer to this question depends on many factors. The largest factor being the time and money they are willing to put into the calibration process. Yet, the NIRS community has stock answers such as "the model can only be as good as the reference method," or "the model can be better than the reference method if you keep only the well-predicted values ${ }^{\prime 11}$. As accurate as these statements might be, they are less than useful for someone without a background in NIRS. 


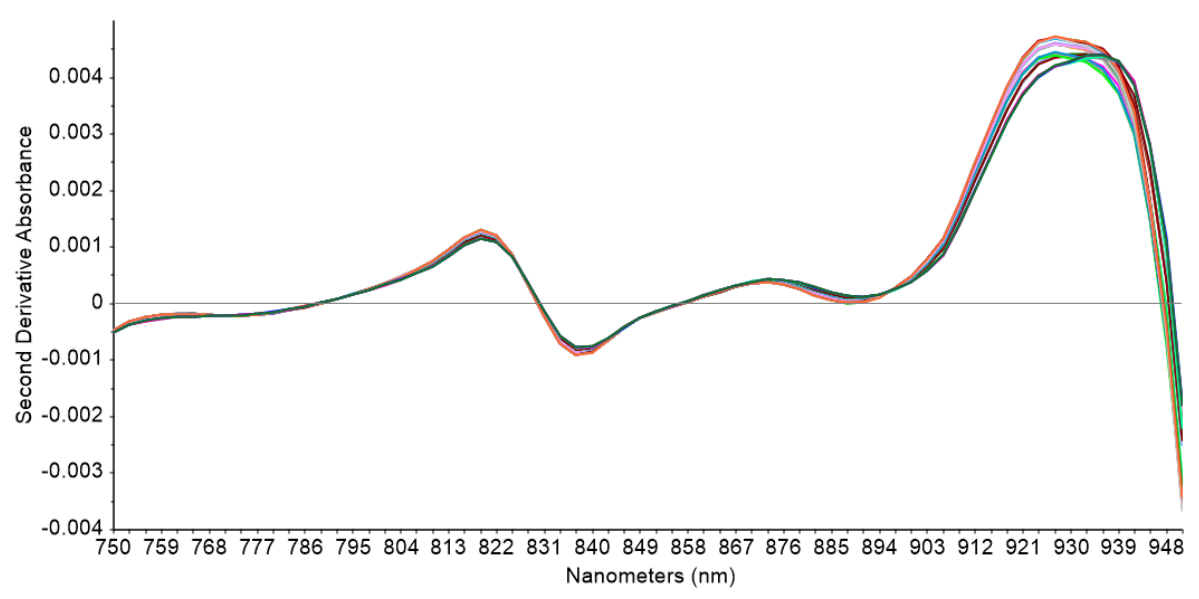

Figure 3.2. Interpolated 9 point Savitzky-Golay second derivative transflection spectra of sugar water in a $5 \mathrm{~cm}$ cuvette, Color code corresponds to the "Actual" reference values in Table 3.1. Spectra collected with Version 1.0 F-750 Produce Quality Meter (Felix Instruments, USA).

The transflection sugar water data in Table 3.1 can be used to better visualize the importance of the reference method accuracy and why it is important to not cut corners while developing a model. By taking a set of 18 spectra, shown in Figure 3.2, and inputting either the estimated sugar concentration or the actual sugar concentration (measured in ${ }^{\circ}$ Brix) into a Partial Least Squares Regression, PLSR, the impact of error in the reference values can be directly measured. The estimated sugar concentration was the target concentration when making sugar water solutions. The actual sugar water concentration was determined by a digital refractometer. From the results shown in Figure 3.3, it becomes obvious that the error of estimated sugar concentration reference values is carried over to the calibration. Specifically, that the Root Mean Square Error of Cross Validation, RMSECV, is nearly double for calibration built with the estimated sugar concentration to that of the actual sugar concentration. Both calibrations were built using the spectral window of 729-975nm, with 2 Principle Components and leave-one-out cross-validation.

\begin{tabular}{|l|l|l|l|l|l|l|l|l|l|}
\hline Specimen \# & 1 & 2 & 3 & 4 & 5 & 6 & 7 & 8 & 9 \\
\hline Estimated ${ }^{\circ}$ Brix Concentration & 2 & 4 & 6 & 8 & 10 & 12 & 14 & 16 & 18 \\
\hline Actual ${ }^{\circ}$ Brix Concentration & 2.1 & 4.2 & 6.5 & 8 & 9.2 & 10.4 & 12.7 & 13.9 & 16 \\
\hline
\end{tabular}

Table 3.1. Comparison of sugar water concentration (measured in ${ }^{\circ}$ Brix) reference values used in a PLSR model. Nine solutions were created, the estimated concentration was the desired concentration when making the solution, the actual concentration of sugar water solution was measured with a refractometer. 

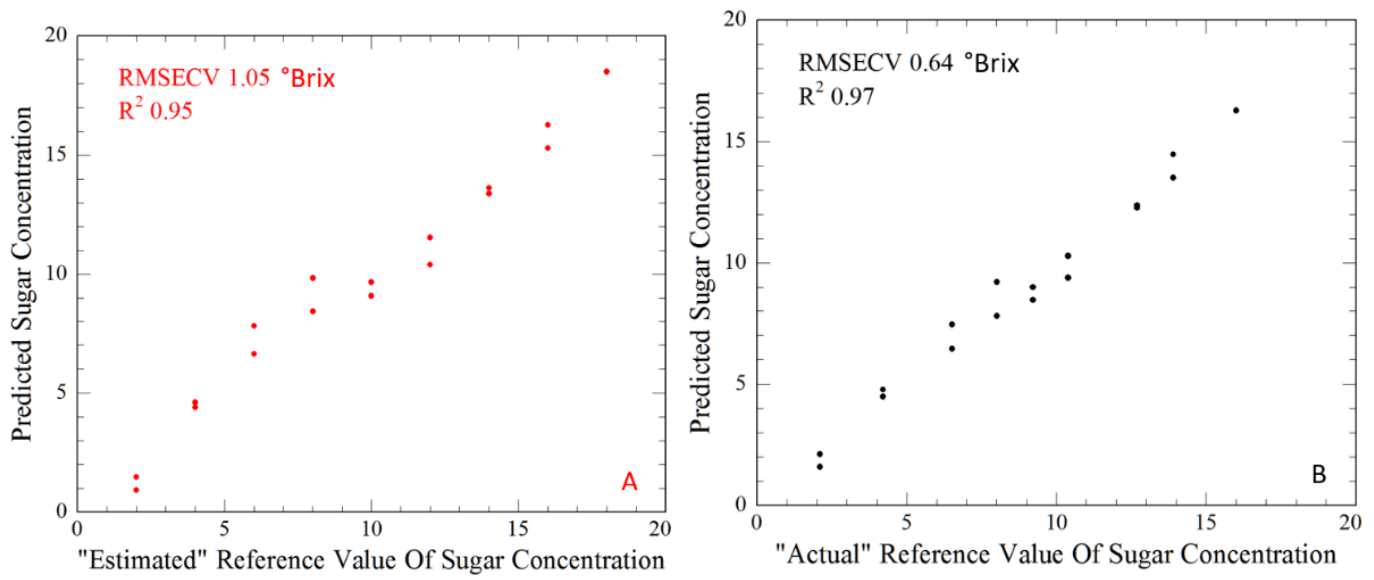

Figure 3.3. Predicted vs Reference graph for A) the estimated ${ }^{\circ}$ Brix concentration B) Actual ${ }^{\circ}$ Brix concentration reference values. 


\subsubsection{Practical Constraints on Sampling and Population Selection for PLSR in Agriculture}

In the author's experience, another question an NIR Operator often asks is "how much fruit do I have to sacrifice for the model calibration process?" In other words, "how big of a population or sample size do I need?" In this case, the stock NIRS community answer is "the model population is large enough when it spans all of the variation in the population, which is determined by adding additional samples to the calibration until the cross-validation results do not improve." Again, this is a great answer for a scientist, but the pragmatic farmer looking to adopt NIR technology needs a more exact answer. To better facilitate the adoption of NIRS into new markets, the practitioners of nearinfrared spectroscopy need to cultivate a language that diminishes the knowledge hurdle and provides practical guidelines that a NIR Operator can implement. The author has found that a simple solution is to suggest collecting 20 samples every week for 5-6 weeks before the expected harvest date. The discussion of how to sample the orchard, tree, or branch, outside the scope of this work. This is necessary not only to capture the maturity point the farmer needs to decide but also to have sufficient spectral and sample variation. Given the limited range of naturally occurring sugar content in fruit for the PLSR to operate, a coefficient of variation greater than $16 \%$ is often required for a complex sample such as an intact fruit (derivation shown below). If the layperson wants a sufficiently stable and accurate calibration, then the coefficient of determination must be high. Equation 9 relates the coefficient of determination to the standard error of the calibration and the standard deviation of the reference samples. Assuming the lowest 
possible error in the reference method is the standard error of the calibration, then the standard deviation of the reference data becomes the limiting factor in calibration performance.

$R^{2}=1-\frac{(S E C)^{2}}{\left(\sigma_{y}\right)^{2}}$

Where $\mathrm{R}^{2}$ is the coefficient of determination, SEC is the standard error of calibration, and $\sigma$ is the standard deviation of the reference value.

Theoretical population statistics can be inferred by applying equation 9 to, for example, a theoretical population of apples. For a trait such as ${ }^{\circ}$ Brix (mg of sugar per $100 \mathrm{~mL}$ of water) in apples, the naturally occurring concentration ranges from $\sim 8{ }^{\circ}$ Brix for a Granny Smith to $\sim 16^{\circ}$ Brix for a Honey Crisp. If the population of apples used for the calibration has a normal distribution, then the average would be approximately $12^{\circ} \mathrm{Brix}$. If the standard error of the calibration is assumed to be $0.6^{\circ} \mathrm{Brix}$, as found in section 3.1.3 for a binary sugar water mixture, which is roughly triple the reported uncertainty of $\pm 0.2^{\circ}$ Brix for a typical digital refractometer, then by plugging these assumptions in equation 9, along with a target coefficient of determination of 0.9 , results in a necessary standard deviation of 1.9 and a coefficient of variation of approximately $16 \%$. This thought experiment is empirically backed by the results presented in Figure 3.18. A simple thought experiment such as the one above results in direct and simple feedback that can be used to explain to a pragmatic farmer exactly what is required to generate a calibration they can use to measure the maturity of their fruit in the field. 


\subsubsection{Introduction to Optical Modes}

Schaare et al. ${ }^{72}$ investigated three modes of visible-near infrared spectroscopic measurement (reflectance, interactance, and transmission shown in Figure 3.4) in order to non-destructively estimate harvest soluble solids content (SSC), density and internal flesh color of yellow-fleshed kiwifruit. Schaare found that an interactance design, comparable to the shadow probe design used in this study, was most accurate for the analysis of sugar content in kiwi fruit. Tsuyoshi Temma et al. ${ }^{73}$ also present an interactance probe instrument for the portable assessment of sugar content. Malinen et al. ${ }^{74}$ presented the design of a transmission-based portable instrument with the use of an LED illumination source source but did not show any application results.

(a) Reflectance

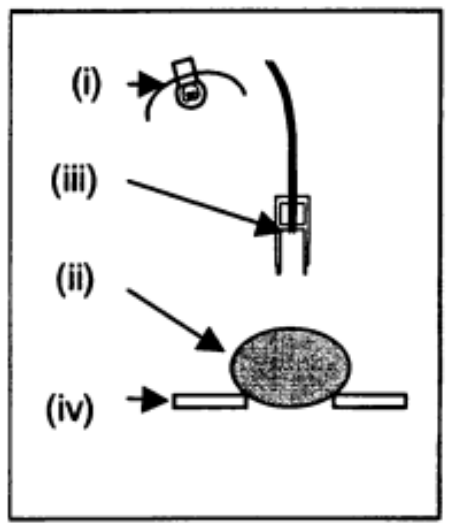

(b) Transmittance

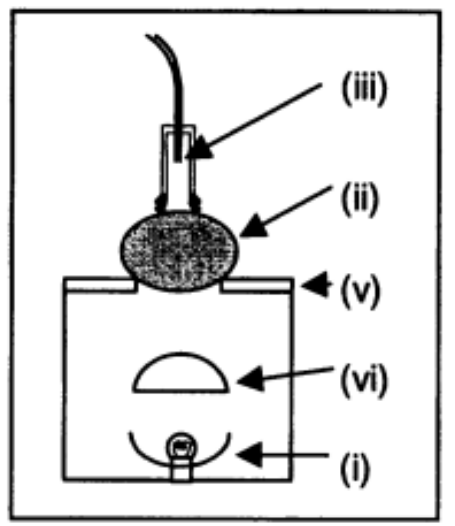

(c) Interactance

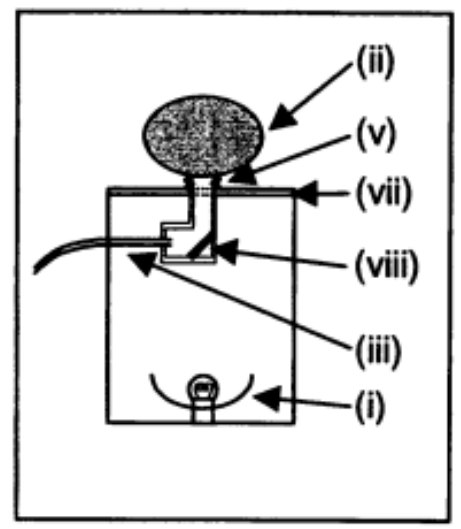

Figure 3.4. Optical modes used in NIR Spectroscopy of Fresh Produce. The apparatus used for measuring (a) reflectance; (b) transmittance; and (c) interactance spectra of kiwifruit, showing (i) the light source; (ii) fruit; (iii) fibre bundle aperture; (iv) black foam holder; (v) light seal; (vi) condenser lens; (vii) glass top; and (viii) mirror. $^{72}$ 
Yuan et al. ${ }^{75}$ present design considerations for a partial transmission design that successfully measures the sugar content of apples. Commercial offerings for portable spectrometer systems that specifically target fruit includes the Sunforest $\mathrm{H}-100$ (Korea), SCiO Pocket Spectrometer (Israel), and Felix Instruments' (USA) F-750. The H-100 employs a partial transmission arrangement to collect spectral information from the cheek tissue of fruits. The SCiO employs a $180^{\circ}$ reflectance arrangement with an LED source. The F-750 employs a shadow probe design. The shadow probe design is a variation of a traditional bifurcated fiber interactance probe. Other commercial offerings for portable spectrometer systems include Viava's (USA) MicroNIR, which employs a $180^{\circ}$ reflectance arrangement; and ASD's (USA) FieldSpec, which employs an interactance probe design.

\subsubsection{Types of Reflectance Rays from a Sample}

Traditionally specular and diffuse reflectance are defined as scattering from a medium such as a powder. As shown in Figure 3.5 the incident rays (yellow rays) which reflect off the surface of the powder undergo specular reflection (blue ray) and incident rays which scatter several layers into the fictitious sample undergo diffuse reflectance (green rays). Whereas, an interactance spectra path, as shown in Figure 3.6 (red rays), undergoes a path similar to transflection reaching 1 to 2 centimeters into the sample before scattering backwards in the direction of the incident light. This form of scattering from deep within the sample is necessary for applications where one wants to see through 
the surface of a sample and collect information on the interior of the sample. As such, the shadow probe design used in this study is ideal for seeing through the skin of fruit and measuring the properties of the edible flesh.

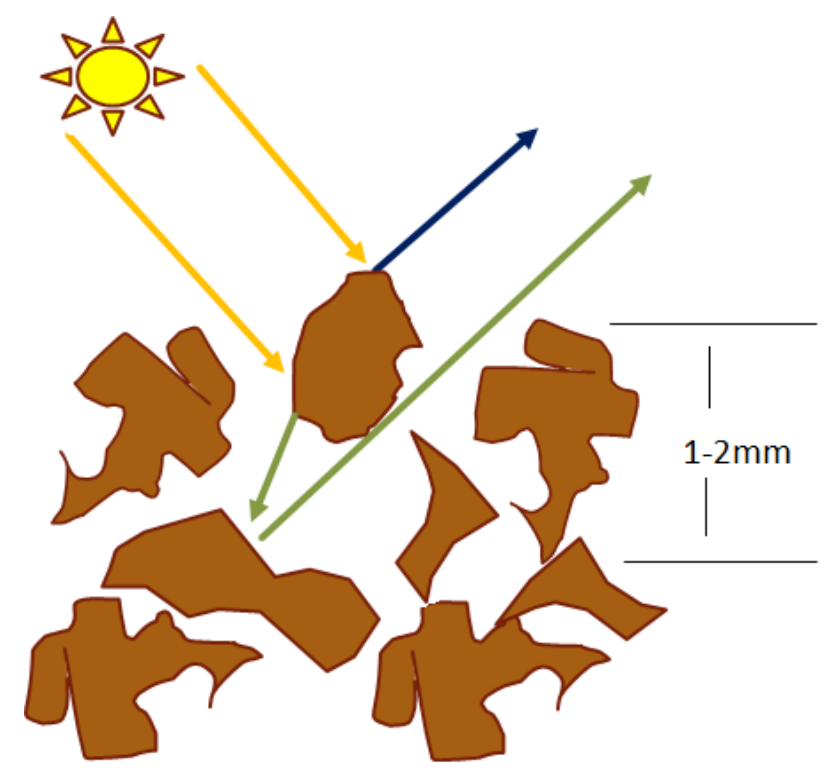

Figure 3.5. Specular reflection, shown in blue, and diffuse reflection, shown in green, rays scattering from a fictitious sample.

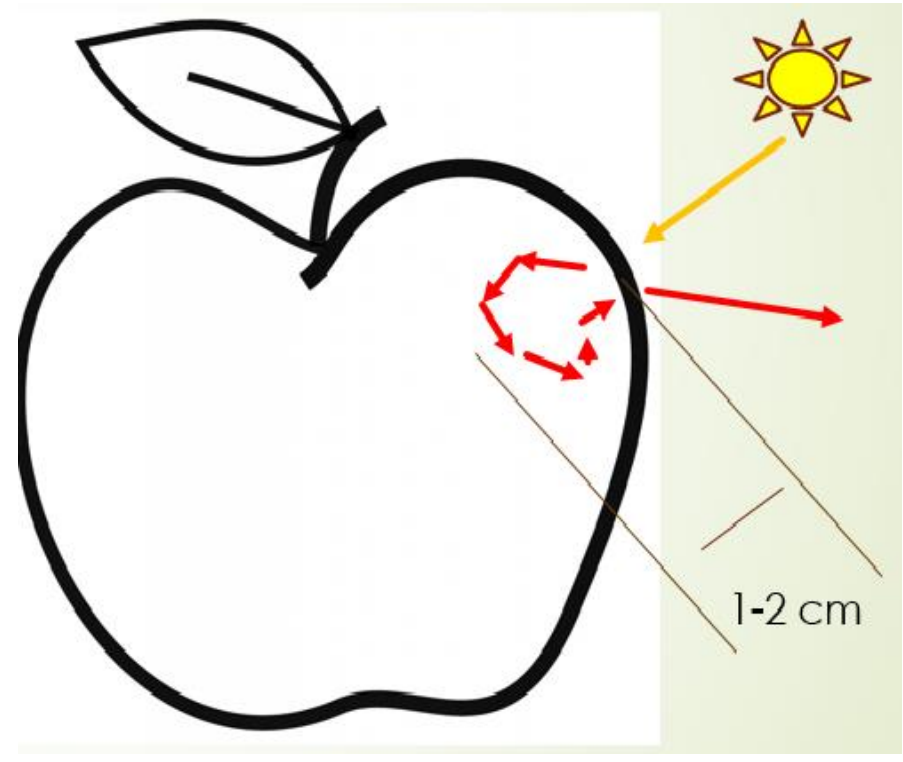

Figure 3.6. Interactance spectra path or a body transflection path, shown in red, scattering internally $1-2$ centimeters into an apple and then reemerging. 


\subsubsection{Why use Shortwave Near Infrared Spectroscopy?}

When designing a spectrometer solution for postharvest applications, the wavelength range is of primary concern. Care must be taken to select a wavelength range that includes absorption peaks of interest and sufficient path length to probe the edible tissue. The use of an FT-IR spectrometer in the range of 1.4 to 3 microns is often employed in the grain industry to identify moisture and protein content of wheat. However, when applied to fresh produce, which consists mostly of water, the issue of signal attenuation, as shown in Figure 3.7, due to water beyond 1.4 microns and below

360 nanometers becomes a concern. Hale et. al. ${ }^{76}$ found that the extinction coefficients of water for visible light is $\sim 10^{-8}$ per meter, whereas the UV and NIR regions are $\sim 10^{-4}$ per meter. Moreover, the wavelength range between $500-1000 \mathrm{~nm}$ has a penetration depth, which ranges from 1-2 cm into fruit. By selecting a silicon-based detector, pigment information such as chlorophyll can be collected alongside the $4^{\text {th }}$ overtone of water at $730 \mathrm{~nm}$, the $\mathrm{CH}_{x}$ overtones between $750-900 \mathrm{~nm}$, the third overtone of $\mathrm{R}-\mathrm{OH}$ at $940 \mathrm{~nm}$, and the $3^{\text {rd }}$ overtone of water at $950 \mathrm{~nm}$. These absorption peaks are advantageous for spectroscopy of fruit as sugar content and other traits of interest contain these moieties. Figure 3.8 displays absorption peaks of interest in the NIR region. 


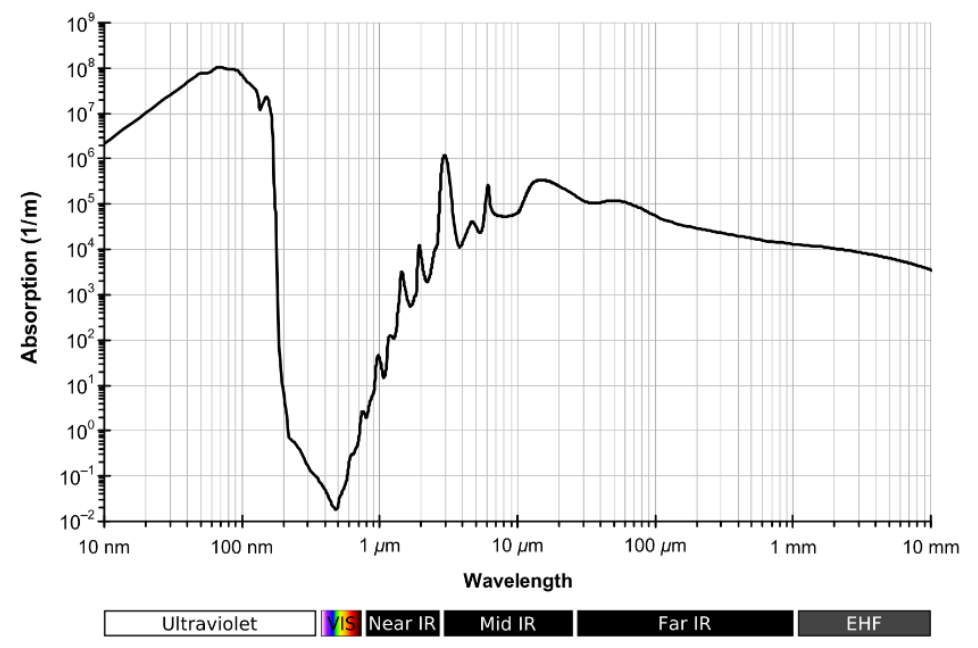

Figure 3.7. Absorption profile of water ${ }^{77}$.

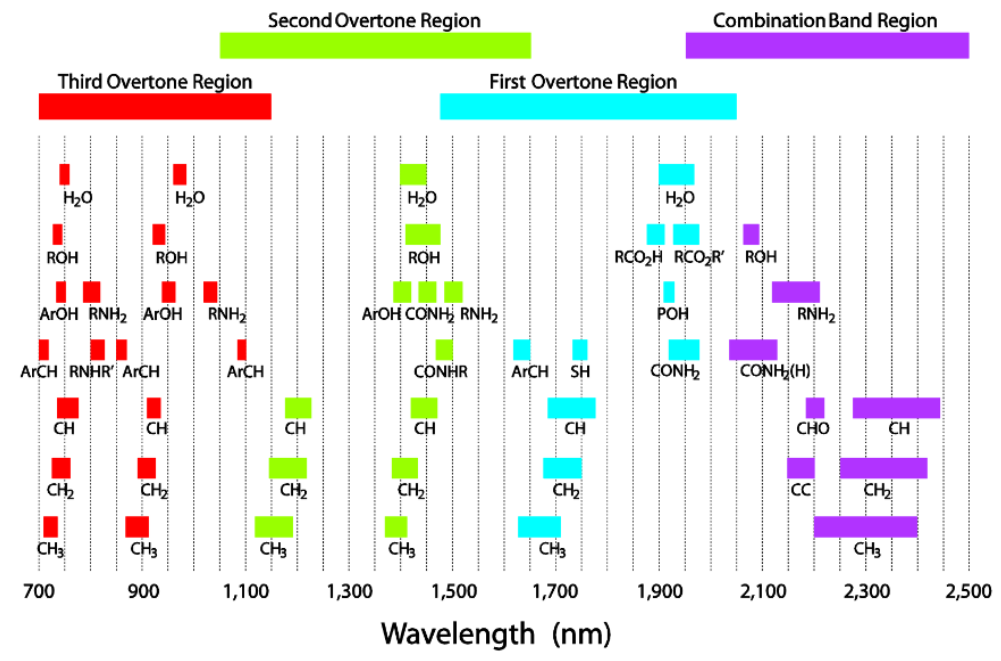

Figure 3.8. Chart of NIR absorption bands ${ }^{78}$.

\subsubsection{Experiment for Approximation of Penetration Depth into Cantaloupe}

Qualitatively the penetration depth of the shadow probe design into melons can be approximated by taking repeated scans of a melon as successive layers of the tissue on the opposite side of the fruit from the spectrometer are removed, as indicated in Figure 
3.9. Once the spectral signature is visually altered, it can be inferred that some portion of the removed tissue was part of the photon-tissue interaction volume in the previous measurements. As such, this experiment allows for the inference of the total path length into a melon.

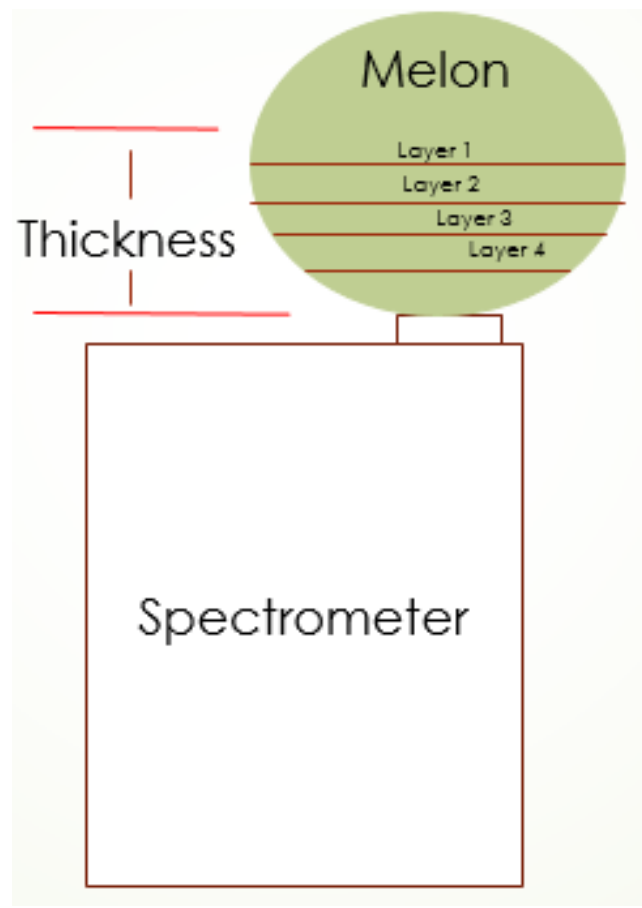

Figure 3.9. Experimental setup for the qualitative measurement of path length. Layer 1 represents $1 / 2$ of a cantaloupe. Layers 2,3 , and 4 are approximately $5 \mathrm{~mm}$ thick.

Figure 3.10 displays the change in the spectral signature as a cantaloupe is reduced in thickness from an intact cantaloupe: half cantaloupe, $21 \mathrm{~mm}$ thick slice, $14 \mathrm{~mm}$ thick slice, $11.1 \mathrm{~mm}$ thick slice. To avoid sample presentation issues, the scanned surface of the cantaloupe was marked with a pen and care was taken to repeatedly place the marked surface of the cantaloupe precisely onto the spectrometer eyepiece. This experiment suggests that the spectrometer can see through the exocarp to a depth 
between 1.4 and $2 \mathrm{~cm}$ into the melon as indicated by the change in the peak height at 937 $\mathrm{nm}$ corresponding to the shoulder of the free water absorption peak in second derivative spectra.

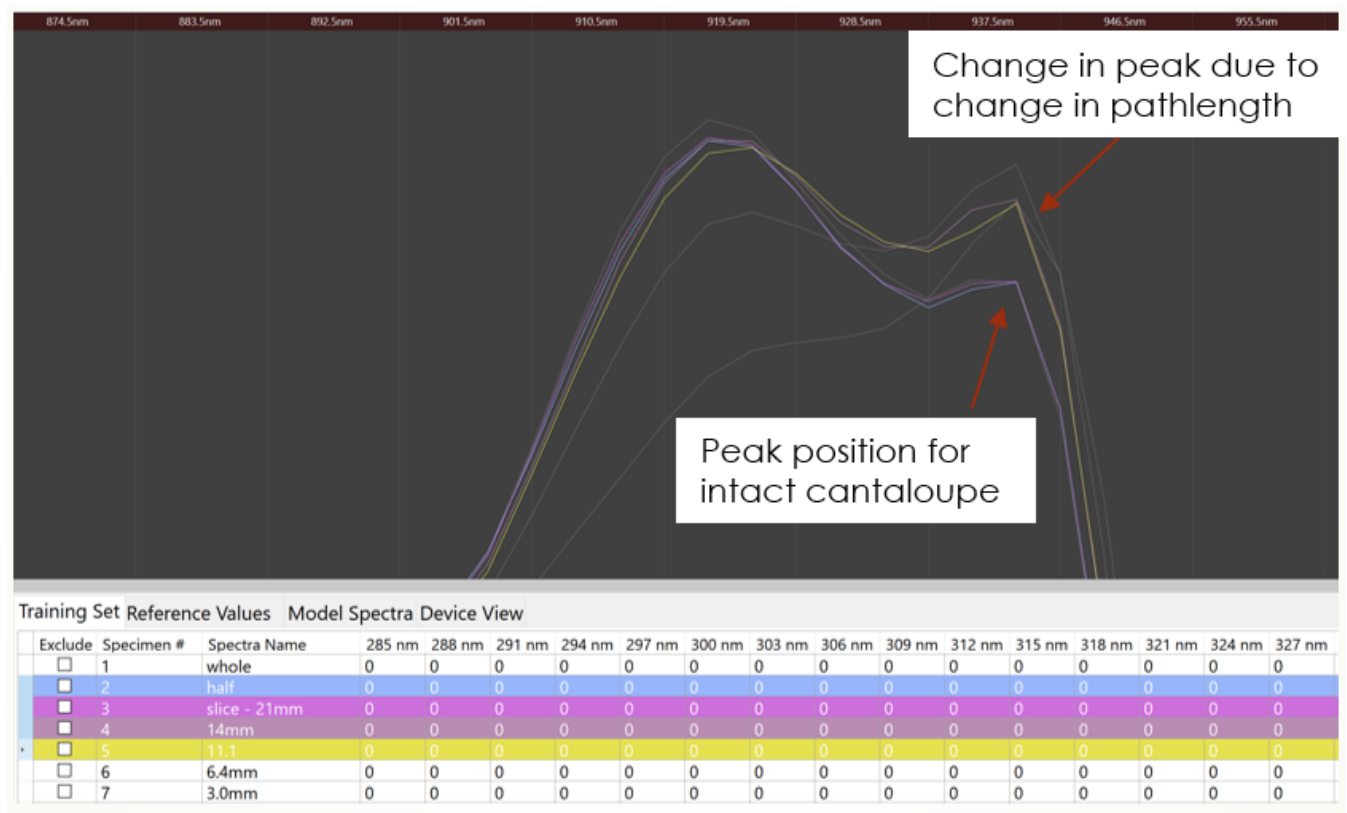

Figure 3.10. The yellow and deep purple spectral responses indicate show a change in peak height at $937 \mathrm{~nm}$ due to the reduction in path length after reducing the cantaloupe to a thickness of $14 \mathrm{~mm}$ (rind and edible tissue). The light blue and magenta spectral responses show common peak height for an approximately half of cantaloupe thick slice and a $21 \mathrm{~mm}$ rind and edible tissue thick slice. A non-colored spectral line corresponding to a whole cantaloupe has the same response as the half cantaloupe slice.

\subsection{The Influence of Dynamic Environmental Factors}

As discussed by Clancy, ${ }^{79}$ the design of a portable spectrometer for field use requires corrections for dynamic operating conditions, such as variation in environmental temperature and external lighting. A theoretical model is presented in chapter 4 with implications of thermal effects on the emission profile of a tungsten lamp and on the reproducibility of spectra collected by a Zeiss MMS-1 spectrometer. External light can be 
accounted by means of a background scan with the illumination source turned off. The spectral response of a sample is also subject to variation in spectral response due to environmental factors. ${ }^{80}$ In addition to mathematical corrections, ${ }^{81,82}$ variation in the sample temperature can be included as a parameter in the design of the calibration space. By inclusion of temperature in the design space, Partial Least Squares Regression is used to separate the covariance of spectral changes due to temperature variation from that of changes in the analyte concentration and successfully predict the analyte of interest regardless of sample temperature.

\subsection{Optimizing the sample probe distance}

\subsubsection{Introduction}

Shortwave NIR can be used in prediction of fruit attributes, such as dry matter, sugar content, or when applicable, internal flesh color on the tree ${ }^{83}$. Common limitations to serving this market have been a lack of true portability, failure to reference for changes in ambient light, and temperature under field conditions (orchard environment). The spectrometer developed in this study is based on the shadow probe spectrometer configuration, as shown in Figure 3.14. As a first principles test of an initial hardware design, sucrose solutions were evaluated in transflection mode, as shown in Figure 3.11. It was assumed that sugar water is analogous to an apple. A similar experiment was performed by Greensill ${ }^{84}$, who found that a wavelength resolution (FWHM) of $10 \mathrm{~nm}$ and a repeatability of 5000 (mean to SD of counts) is sufficient for the determination of sugar in water. This requirement is met by the Zeiss MMS1 spectrometer. 


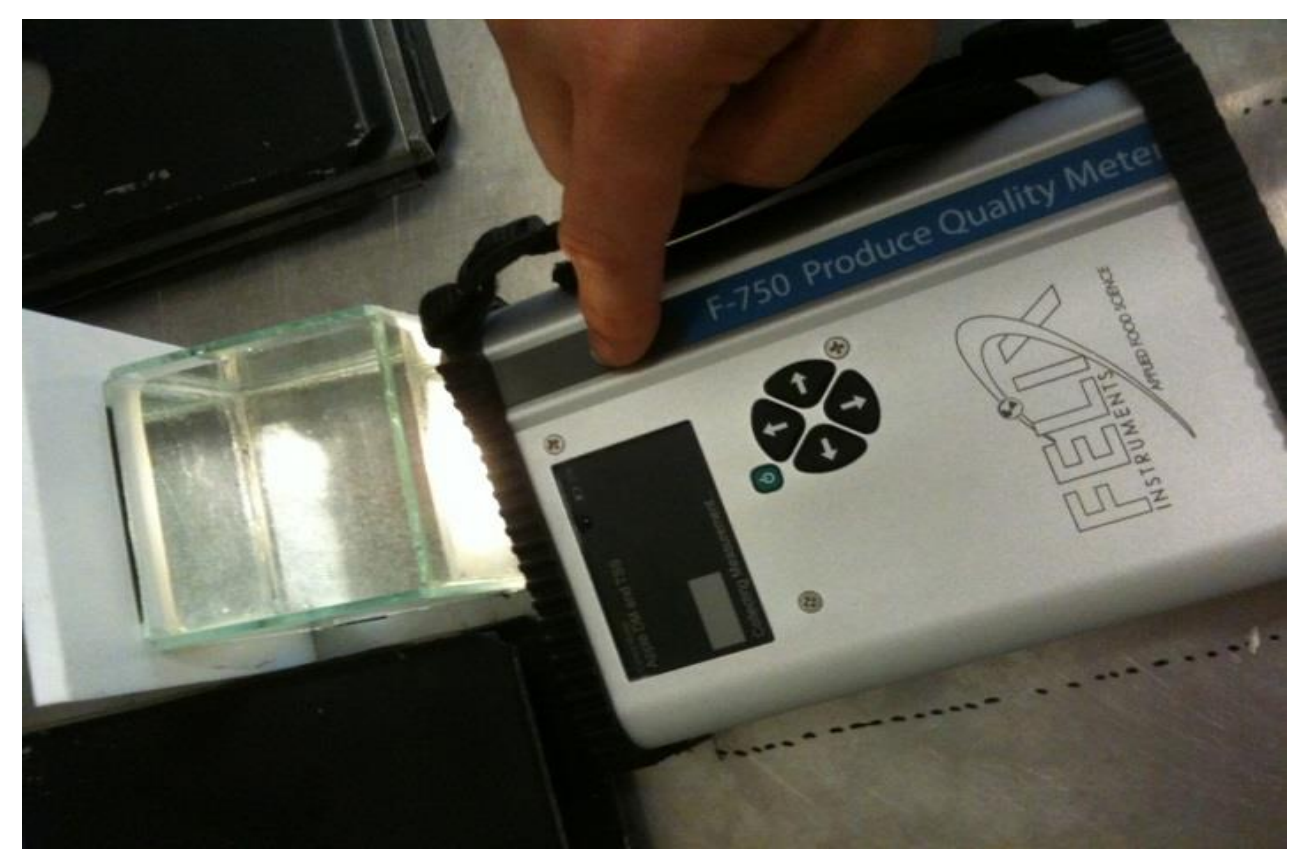

Figure 3.11. The prototype shadow probe spectrometer collecting sugar water spectra. As predicted by the requirements of Greensill ${ }^{84}$, the spectra collected by the instrument was correlated by Partial Least Squares Regression with the concentrations of sucrose solutions. When the hardware was later tested against a population of ready-to-eat apples, Partial Least Squares Regression failed to find a correlation with the dry matter values. The output of the Partial Least Squares Regression for the sugar water and apple experiments are shown in Figure 3.12. The Dry Matter values were collected following the standard operating procedure provided by Dr. Phul Subedi of Central Queensland University ${ }^{85}$. The Zeiss MMS1 and shadow probe design were first used in the Nirvana (Spectrophotonics, Australia) to successfully predict the internal traits of mangoes ${ }^{86}$. This suggested that a hardware design difference in the optical bench of the shadow 
probe design used in this study was preventing the instrument from collecting apple spectra that would correlate with the dry matter values.
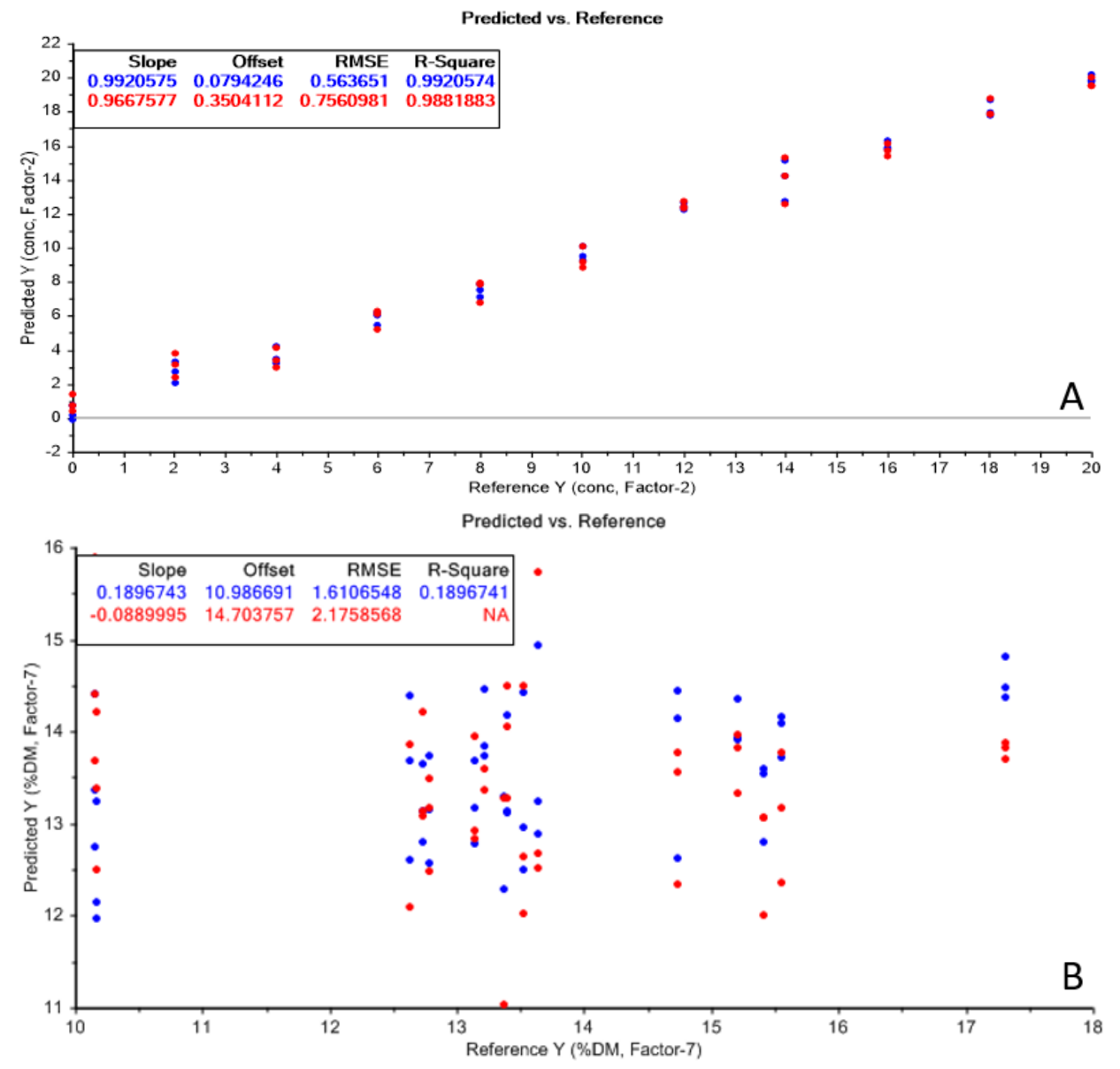

Figure 3.12. Contrasting the performance for a sugar water transflection experiment, which is thought to be a first approximation of an apple, and an apple dry matter calibration A) A successful two factor PLS model generated with Unscrambler 10.3 for sugar water transflection. Leave-one-out cross-validation is marked red. B) Despite the inclusion of seven factors, PLS model generated with Unscrambler 10.3 for Dry Matter of Apples was not successful.

An experiment by Walsh et al. ${ }^{87}$ evaluated the ratio of specular to diffuse reflectance in a shadow probe design by placing a polarizer on the light source as well as on the detector input. Subsequently, spectra of an apple and kiwi fruit were acquired as the sample to probe distance was varied in $10 \mathrm{~mm}$ steps from 0 to $150 \mathrm{~mm}$ (Figure 3.13). 
The ratio of specular to diffuse reflectance is presented in Figure 3.13. At probe-tosample distances $<30 \mathrm{~mm}$, the ratio of specular-to-diffuse radiation was found to be $<$ $3 \%$. The specular component increased rapidly at greater distances due to loss of the shadowing effect. Additional work on shadow probe design for inline grading of fruit (Packhouse sorting machines) can be found in the unpublished thesis of Clinton Hayes ${ }^{33}$. Specular reflectance is assumed to only contain information regarding the surface of the fruit. Diffuse reflectance is assumed to contain information about the internal edible tissue. As such, a shadow probe design should seek to minimize the amount of specular reflectance collected by the probe.

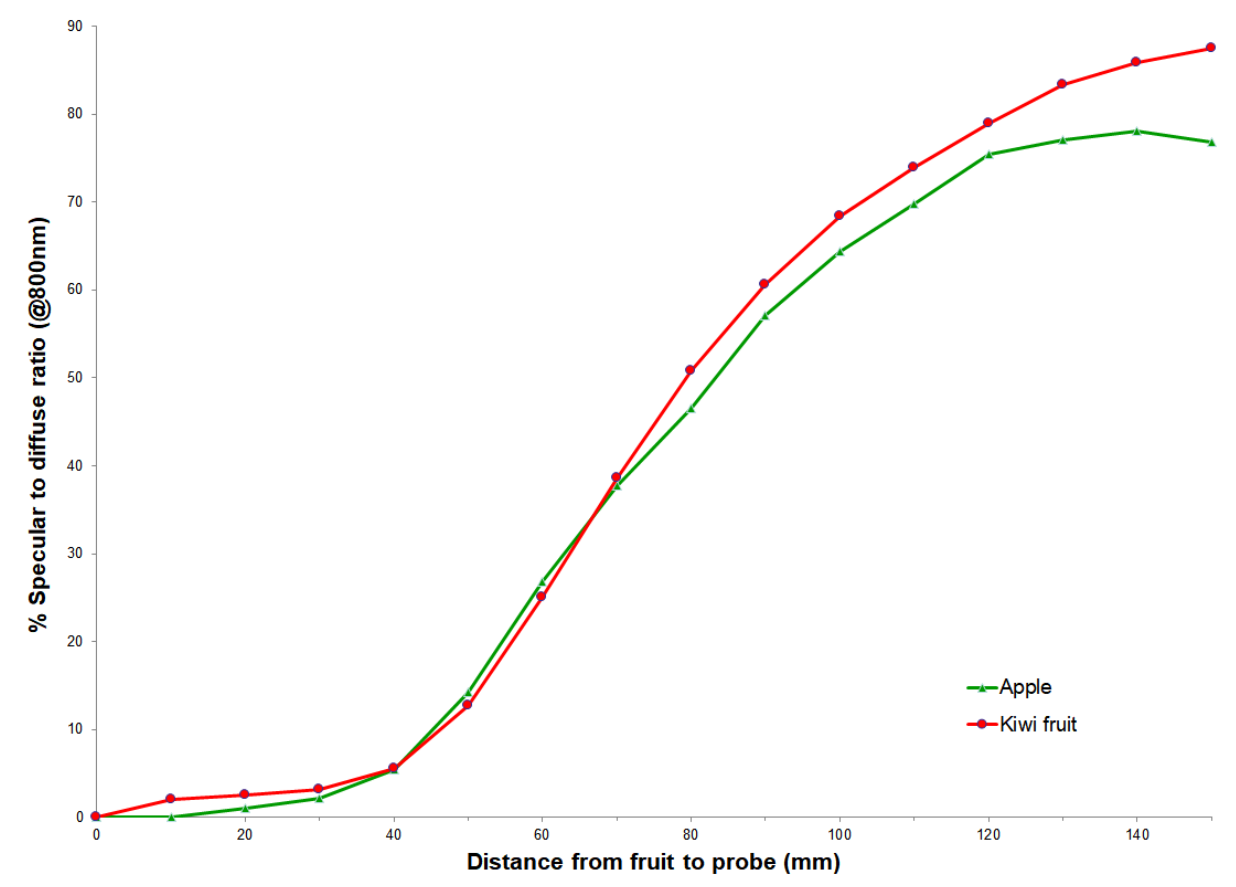

Figure 3.13. The ratio of specular to diffuse radiation received by the shadow probe from an apple and a kiwi fruit, estimated using polarized illuminating light and a cross polarizer on the detector probe, to separate specular from diffusely scattered light. The amount of specular reflections collected by the spectrometer increases with sample-to-probe distance ${ }^{87}$. 


\subsubsection{Experimental Setup}

The experiment presented by Walsh et al. ${ }^{87}$ lacks resolution for the sample-to-probe distances used in portable handheld equipment. To address this, an experiment was conducted that varied the sample-to-probe distance in $5 \mathrm{~mm}$ steps between 0 and 25 $\mathrm{mm}$. Because fruits are expected to reflect a mixture of specular and diffuse reflectance, polarizers were not used in this experiment. Instead, a ceramic tile was used as a specular reflectance standard and a Teflon block provided a combination of specular and diffuse reflectance. A halogen lamp within a parabolic reflector produced light that was 'guided' to the sample through a $32 \mathrm{~mm}$ diameter aluminum tube. Possible light paths between the lamp, sample, and then to the lens are shown in Figure 3.14. The red path in Figure 3.14, demonstrates that reflections off the tube wall could produce specular reflectance on the sample surface. Based on this model, it was hypothesized that the apple experiment failed because signals being collected by the spectrometer may, in fact, contain little information of the internal tissue. Instead, the apple signal being collected may be primarily comprised of specular or skin information due to too large of a sample-to-probe distance.

To investigate and minimize the inherent spectral reflectance from the tube, three variations of the collimating tube with different surface finishes were evaluated as a function of the sample-to-probe distance. The "regular" tube had no special processing done after extrusion. The "polished" tube was hand polished for 40 minute with 
aluminum polish. The "half and half" tube was polished at the sample end and painted flat black near the lamp. The test rig is displayed in Figure 3.15. The term "diffuse reflectance" has been used rather loosely. The light collected by the spectrometer is expected to travel up to $2 \mathrm{~cm}$ into the tissue of an apple and then reemerge normal to the collimating lens. No work on the path length for a shadow probe has been published. The assumed path length for fruit is inferred based on the qualitative results shown in section 3.1.8 and an experiment by Greensill, which looked at the attenuation of light transmitted through stacks of filter paper soaked in sugar water ${ }^{84}$. This assumed, $\sim 4 \mathrm{~cm}$ light path does not fit in with the standard model of diffused reflectance, as discussed in section 3.1.6. A more appropriate term when collecting spectra of an apple or other fruits maybe backscattered light from the interactance volume of tissue or simply interactance light.

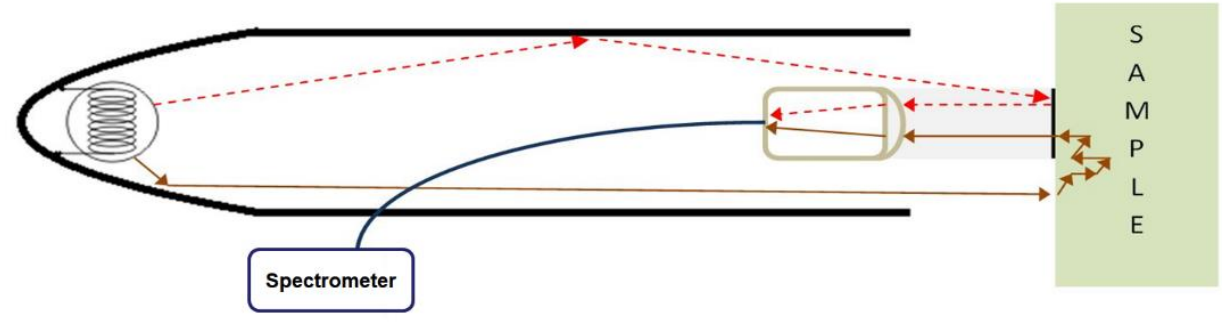

Figure 3.14. 'Shadow probe' design. An interactance ray (brown) undergoes remission normal to the collimating lens and is focused onto the fiber. A specular reflection ray (red) is scattered by the collimating tube in a fashion that results in specular reflectance from the sample being received by the spectrometer. 


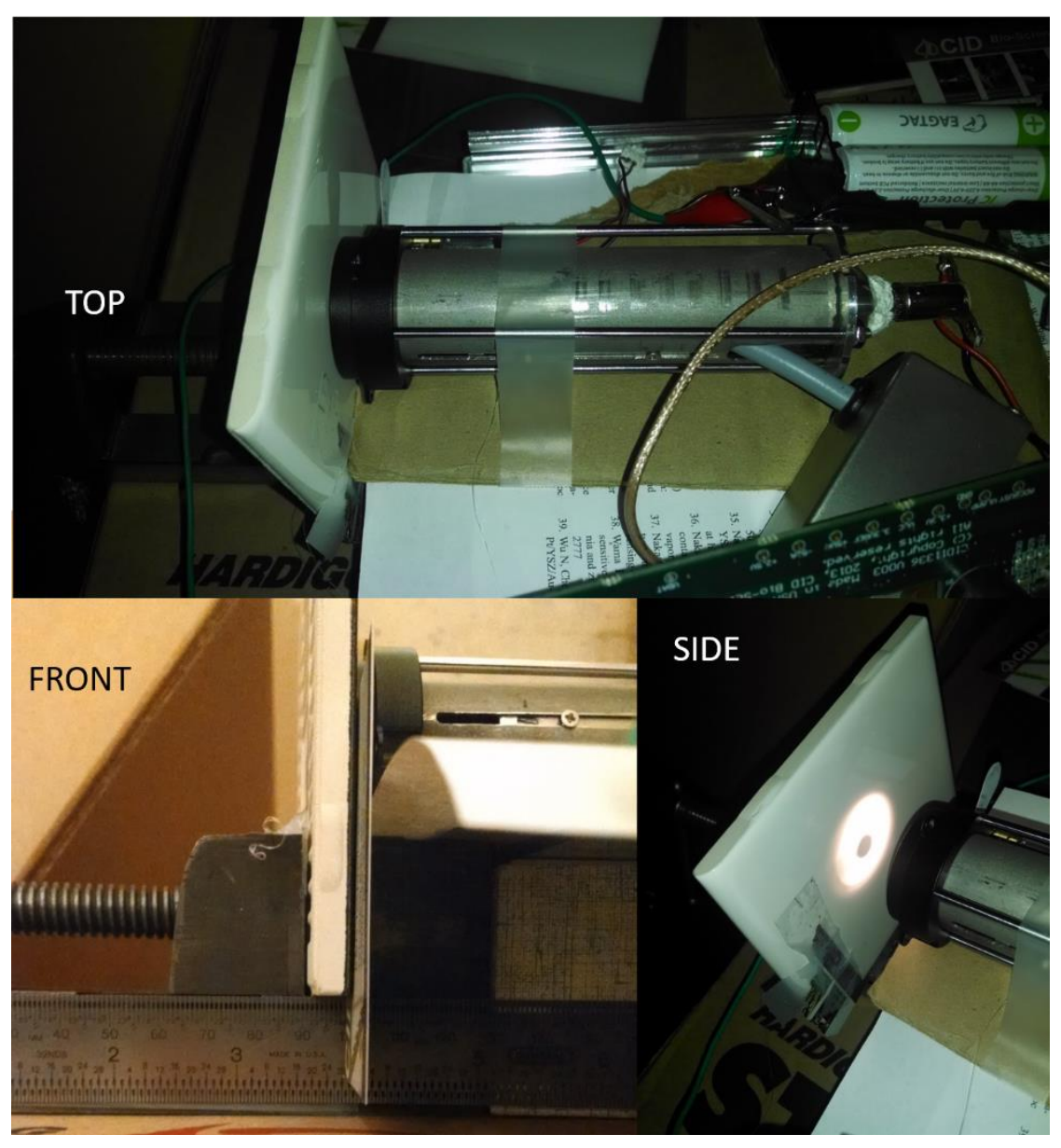

Figure 3.15. Photo of the experimental setup used to measure the influence of probe to sample distance. Top) Fiber bundle from MMS-1 mounted inside of tube. Left) Ruler and position marker for sample to probe distance. Right) Image of the shadow being cast onto the ceramic tile.

\subsubsection{Results}

As the distance between sample and probe increases, the shadow becomes less defined as more light scatters from the sidewall of the tube into the shadow (Figure 3.15), which is consistent with the findings of Walsh et $\mathrm{al}^{87}$. The variation in the shape of the illuminated circles in Figure 3.16 are due to changes in the camera position, exposure time and other parameters which were not precisely controlled. No direct quantification 
of shadow quality was undertaken. An additional experiment could have been performed by placing a series of photodiodes around the illuminated annulus and in the shadow. This additional work was not performed, because the reflectance data of the ceramic and Teflon targets was sufficient to make design changes. The specular reflection from the polished tube $25 \mathrm{~mm}$ away allowed for a clear image of the collimating lens to be projected onto the ceramic target.

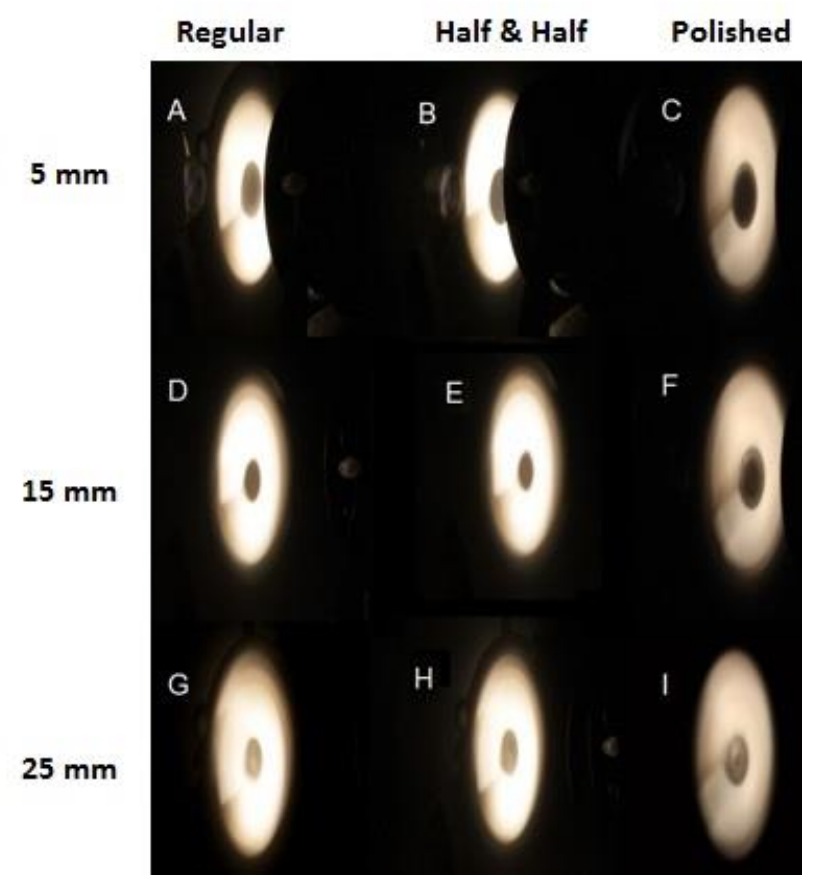

Figure 3.16. The visual shadow cast by the probe onto the white tile. Separation of 5,15 , and 25 $\mathrm{mm}$ displayed in the top, middle and bottom rows respectively, for A, D, G Regular Tube; $\mathrm{B}, \mathrm{E}, \mathrm{H}$ Half-and-Half Tube; and C, F, I Polished Tube. 
The polished finish, with its highly reflective surface, has the largest increase in specular reflectance as a function of distance (Figure 3.17). The half-and-half tube, with its antireflective coating (flat black paint), has a minimal increase in specular reflection at distances between 5 and $20 \mathrm{~mm}$. The $0 \mathrm{~mm}$ data points are not shown because the shadow probe design requires space for an internal white reference to be toggled in between the lens and the sample.

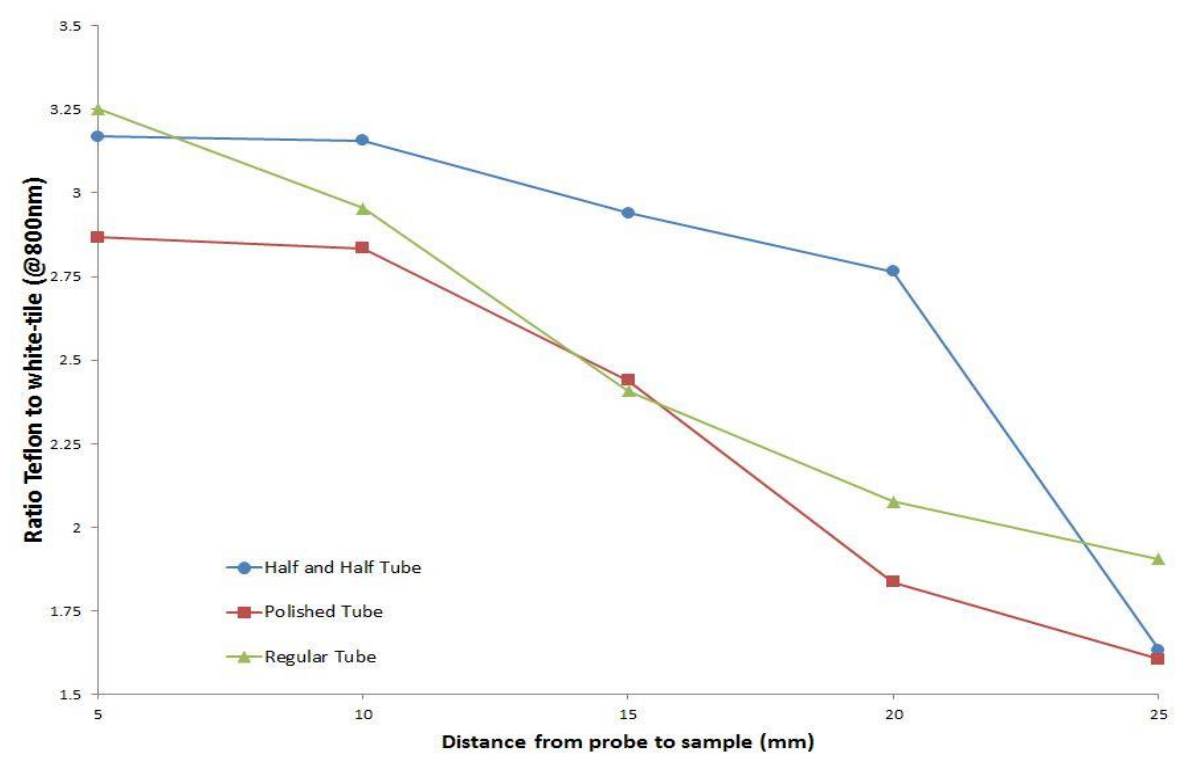

Figure 3.17. The Reflectance ratio of Teflon to ceramic tile as a function of distance and tube finish. The half and half tube had the smallest increase in specular reflection between 5 and 25 $\mathrm{mm}$.

\subsubsection{Conclusion of the collimating tube experiment}

A collimating tube, which is partially painted flat black, reduces the quantity of specular reflection detected in a 'shadow probe' design as compared to an untreated or a highly polished tube, and so produces an apparent stronger shadow on the sample surface. 
Following this work, a sample-to-probe distance of $\sim 15 \mathrm{~mm}$ was selected. This distance allows for the installation of an internal reference shutter into the shadow probe configuration. Upon implementation of the $15 \mathrm{~mm}$ sample-to-probe distance, the shadow probe spectrometer configuration can collect spectra that correlates with the dry matter of a population of apples, shown in Figure 3.18.

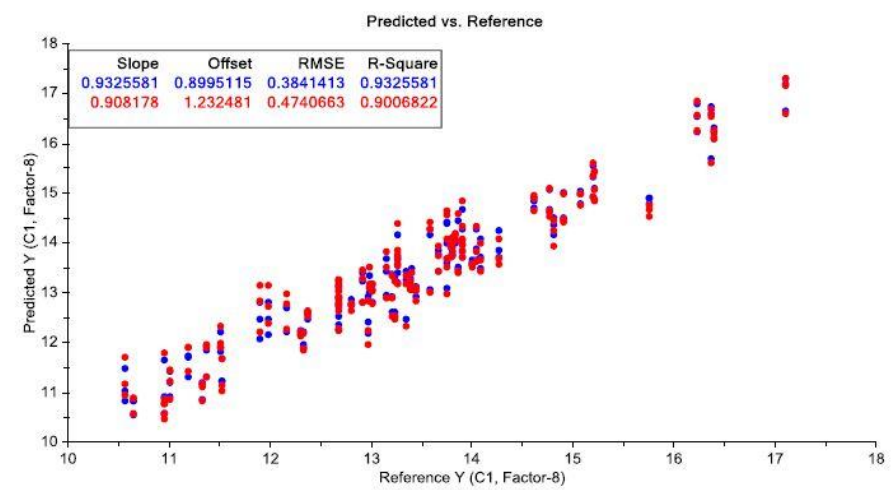

Figure 3.18. An eight factor PLS model generated with Unscrambler 10.3 for Dry Matter of Apples after improving the sample-to-probe distance.

\subsection{Summary}

Near Infrared Spectroscopy can be performed on intact fruit as predicted by BeerLambert law, which assumes that changes in spectra are only due to changes in the analyte of interest. The change in concentration of the analyte of interest can be nondestructively predicted by modeling the relationship between the analyte of interest and the spectral signature of the fruit with multivariate math. Partial Least Squares regression, a multivariate method, does not suffer from the collinearity issues of finding $\left(X^{\prime} X\right)^{-1}$, making it ideal for the analysis of fruit spectra. 
This work demonstrated three factors which influence the ability of an interactance spectrometer to generate a Partial Least Squares regression. The accuracy of a Partial Least Squares regression is influenced by the accuracy of the primary reference method. As demonstrated with transflection sugar water spectra, if an approximation of the sugar concentration is used in a Partial Least Squares regression the uncertainty of the resulting calibration is greater than the Partial Least Squares regression utilizing the true reference values. This factor may seem obvious, but highlights that the fact operator error in collecting the reference data for a regression can limit model statistics regardless of spectrometer hardware performance. Additionally, sample population statistics, such as the standard deviation, have been found to impact the accuracy of a calibration. In the case of in situ sugar concentration of apples, a coefficient of variation greater than $16 \%$ is theoretically required to produce a Partial Least Squares regression with a coefficient of determination of 0.9 . The third factor which has been shown to influence a partial least squares regression is the sample to probe distance. If the sample to probe distance is increased beyond $20 \mathrm{~mm}$, the ratio of specular to diffuse reflectance increases, which comprises the ability of a shortwave NIR spectrometer to see through the skin and into the edible tissue of fruit. This study recommends a sample to probe distance between 10 and $20 \mathrm{~mm}$ for a shadow probe interactance design.

Section 3.1.3 was previously published as:

Lerud, R. (2016). The Importance of the Reference Method Accuracy and Why it is Important Not to Cut Corners While Developing a Model. NIR News, 27(3), 21-22. https://doi.org/10.1255/nirn.1603 


\section{Chapter 4}

The Role of Spectrum Collection Protocols and Reference Scans 


\subsection{Overview of Design Considerations for Handheld Spectrometers}

The following sections cover a variety of issues and disparate experiments related to the collection of high quality spectra from handheld spectrometers. Variations in hardware design and the protocols used to calculate the absorbance spectra have a direct relationship with the repeatability of spectra collected by individual spectrometers. Repeatability is a key metric of hardware performance. The variance in repeated scans can be used to explain the issues presented in Chapter 5 when transferring calibrations between spectrometers. The following work introduces the general concept of noise as unwanted signal and then presents concepts for the reduction of noise in spectrum collection protocols.

All electronics have noise, be it the camera in an iPhone or the pocket spectrometer which can be carried to the grocery store. The engineers involved in an instrument's design have done their best to minimize the noise, but there are always compromises. For example, it is hard to control temperature in a portable battery driven device, wherein active (fan) cooling is often not included to help conserve battery life. In general, this trade-off allows the temperature of the detector to fluctuate due to radiative heat from the lamp, electronics, and environmental ambient temperature.

How temperature variation impacts spectral quality can be inferred from a fundamental understanding of how a spectrometer operates. Light is captured by the optics, with the different wavelengths separated and projected onto an array of photodiodes. Each 
diode acts as an independent detector or pixel by absorbing photons and using their energy to excite electrons to a higher energy level. This photo-excitation process results in the accumulation of electrical charge or voltage at each diode. Light is collected for the duration of the integration time. Once the light exposure is complete, an electrical circuit measures the analog signal by counting total charge at each detector and converts that information into a digital signal, which is read by a computer as a spectrum.

If the optical and the non-optical components of the spectrometer are designed so well that the thermal expansion of materials can be corrected so it does not affect their performance. The correction (equation 10) then allows for the discussion to be focused on the effects of thermal fluctuations to be limited to the illumination source and detector.

$\frac{\Delta L}{L}=\alpha_{L} \Delta T$

where $L$ is the length, $\Delta L$ is the change in length due to thermal expansion, $\alpha$ is the coefficient of expansion, and $\Delta T$ is the change in temperature of the material. 


\subsection{Spectrum Collection Protocols}

A spectrum collection protocol typically includes:

1. Collect a spectrum of the white reference standard with the lamp off. This provides a background or dark correction.

2. Collect a spectrum of the white reference standard with the lamp on. This provides a normalization scan to make the instrument self-consistent.

3. Collect a spectrum of the sample with the lamp on.

4. Collect a spectrum of the sample with the lamp off. This provides a correction for the self-illumination of the target. (external light levels)

These four spectra can be combined as shown in equation 11, to construct a corrected spectrum. Additional factors such as integration time can be included to further normalize the data. Moreover, in the application of NIR and fresh produce, it is often advantageous to apply a $\log _{10}$ transform on reflectance spectra, as shown in equation 12 , to absorbance as part of the protocol. Additional preprocessing such as SavitzkyGolay filters ${ }^{88}$ can be applied to smooth the data and account for baseline shifts prior to multivariate analysis.

Reflectance Spectrum $=\frac{\text { Sample }_{\text {lighton }}-\text { Sample }_{\text {lightoff }}}{\text { WhiteReference }_{\text {lighton }}-\text { WhiteReference }_{\text {lightoff }}}$

Absorbance Spectrum $=-\log _{10}($ Reflectance Spectrum $)$ 


\subsection{Thermal Effects on The Illumination Source}

The Near Infrared (NIR) and chemometric fields have a wealth of literature focusing on spectral signal processing ${ }^{57}$. Standard Normal Variate ${ }^{58}$ (SNV) and Multiplicative Scatter Correction $^{59}$ (MSC) are two signal processing approaches commonly used to pretreat spectra for baseline differences due to path length or scattering effects (both multiplicative and additive). ${ }^{60,61}$ However, there is a scarcity of approaches in the literature on improving spectrometer hardware designs to mitigate the sources of noise that necessitate spectral pretreatment. Advances in hardware design will enable the NIR community to push beyond their current reliance on signal processing as the only method for controlling the non-linear effects currently seen in NIR spectra.

Understanding the effects of source temperature and lamp stability will lead the NIR community to improve spectrum collection protocols. High quality spectra can be acquired by designing the hardware for better control of source filament current, and overall spectrometer temperature stability. Figure 4.1 illustrates the red shift in the emission peak and a decrease in the total flux of a typical tungsten halogen lamp as the temperature of the filament is reduced. This is analogous to how a household dimmer switch reduces the intensity of the light and experiences a red shift as the current is reduced. The household dimmer switch controls the current supplied to the lamp, which adjusts the emitted intensity and filament temperature. 


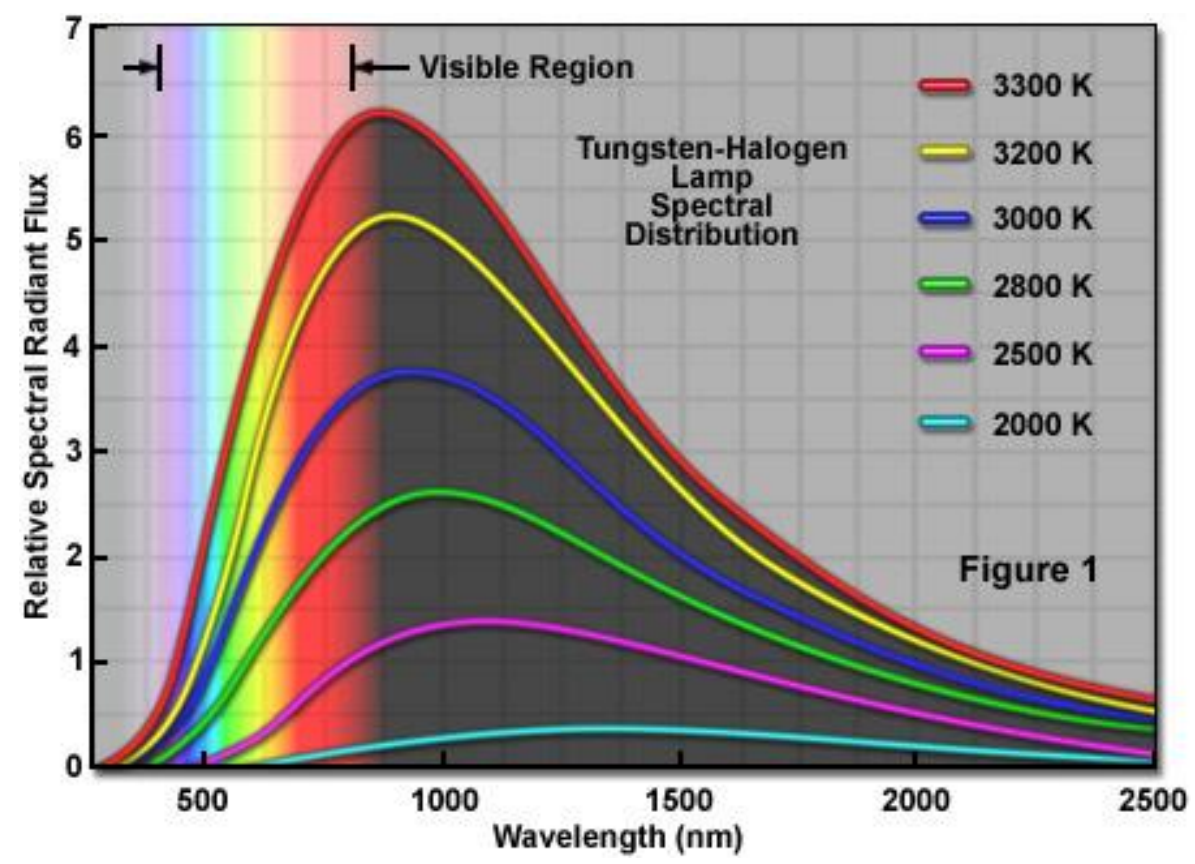

Figure 4.1. Spectrum of typical tungsten halogen lamp. Note not only does the total area under curve increase with increase in the source temperature, but it also induces a blue shift in the peak maximum. ${ }^{89}$

Applying this analogy in reverse, as we turn on a light with a household dimmer switch, the light intensity slowly increases with the increasing current, until both the supplied current and emitted intensity reach equilibrium. To achieve repeatable results with handheld spectrometers, we must also wait for the lamp to be sufficiently stable, meaning that it must reach a constant temperature and current. This is unlike a typical table top UV-vis spectrometer, where the simple solution to achieve stable lamp flux is to turn the lamp on for a few hours prior to use. Handheld spectrometers are battery powered so the lamp must be turned off in between the scans to conserve power and extend its field-use lifetime. As shown by Figure 4.2, it takes approximately $250 \mathrm{~ms}$ for 
the intensity of a xenon tungsten aircraft cabin lamp to become sufficiently stable for repeatable spectra to be collected.

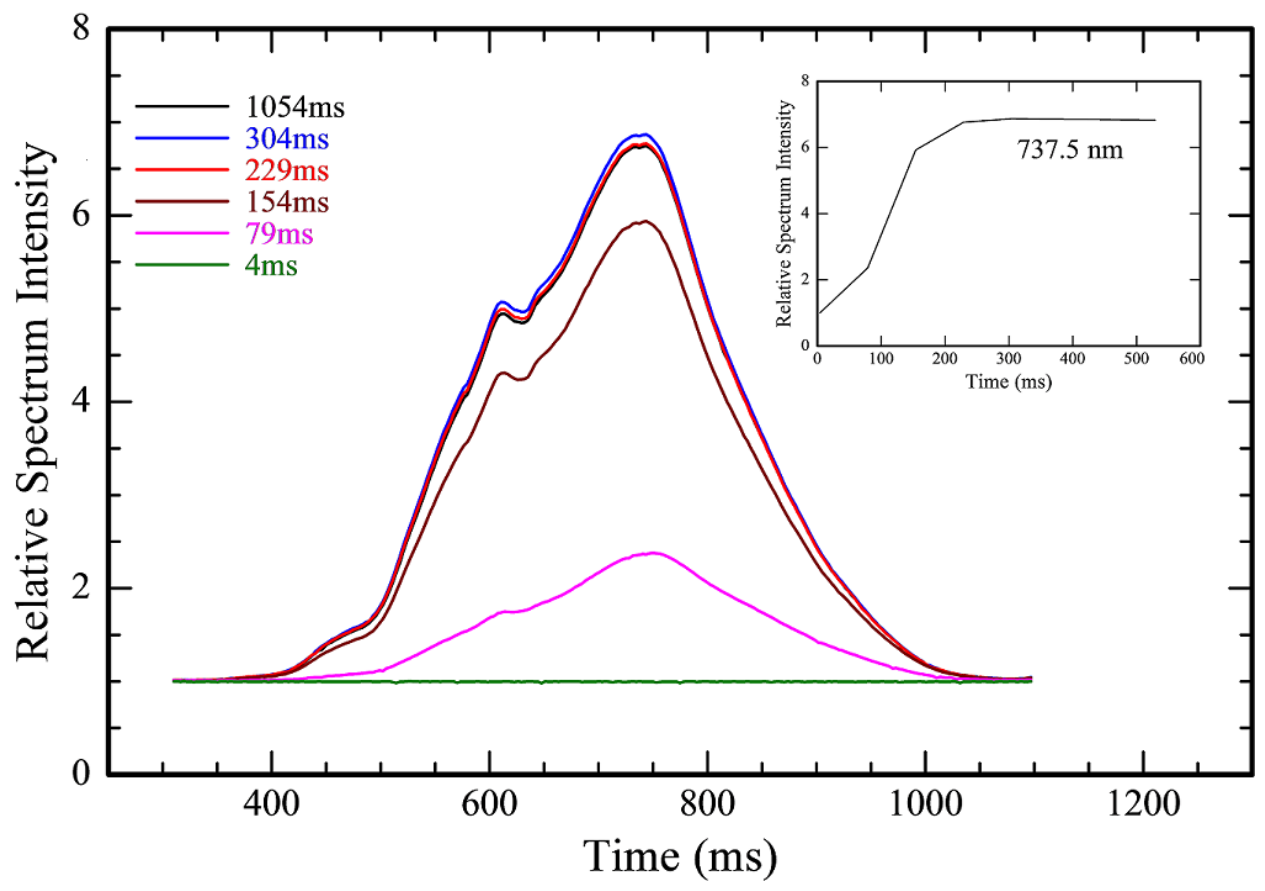

Figure 4.2. Relative intensity of a xenon tungsten lamp collected with a Zeiss MMS-1, immediately after turning on the lamp (20ms integration with a $55 \mathrm{~ms}$ pause between scans). It is important to note that the above thermal behavior is characteristic of the type of light source. The insert is relative intensity at $737.5 \mathrm{~nm}$.

Aside from conserving battery energy, thermal management inside the handheld spectrometer must also be optimized. This is not only to provide comfortable handling of the spectrometer but also to minimize the effects the source temperature variation has on the collected spectra. The heat generated by the lamp has two major effects on device performance. The first is the residual heat from the previous scan, which alters lamp emission performance on the subsequent scan. This is described by the StefanBoltzmann law, which states that the flux of a blackbody emitter varies by quadruple 
power of its temperature. In other words, a small change in the source temperature between scans leads to a large change in the spectrum of the lamp, as shown by the various curves in Figure 4.1. These spectral shifts can be normalized by use of a reference spectrum. The second effect is the dissipation of heat from the lamp to the surrounding spectrometer components. As these internal components warm up, their contribution to the thermal noise in the collected NIR spectra increases. The thermal noise can be partly accounted for by subtracting a lamp off spectrum (background or dark scan). It is important to note that the above thermal behavior shown in Figure 4.2 is characteristic for each type of light source.

\subsection{Thermal Effects on The Detector}

From basic semiconductor physics, we know that temperature variation on the detector primarily manifests itself as dark current; the so-called background noise of the photodiode. In most spectrum collection protocols, this effect is corrected for by taking a scan in the absence of light and subtracting it as an offset from the light exposed scan. A secondary temperature dependent factor of the detector is the responsivity or photosensitivity of the detector, which is proportional to the quantum efficiency of converting photons into excited electrons. A typical spectrum collection protocol assumes that the detector is at a constant temperature and as such can assume that any fluctuation in the quantum efficiency is negligible. 
As shown in Figure 4.3A, the peak photosensitivity of the detector is at $600 \mathrm{~nm}$. Figure 4.3B shows that there is essentially no influence of temperature in the visible range below $600 \mathrm{~nm}$. Within into the shortwave NIR region $(700-1100 \mathrm{~nm})$, the temperature influence the photosensitivity. At $800 \mathrm{~nm}$, the detector has a photosensitivity of approximately 0.17 Amps/Watt (excited electrons/absorbed photons), and a thermal correction of $0.3 \% / C$ (i.e. the detector becomes more photosensitive at this wavelength as temperature increases). For a $5^{\circ} \mathrm{C}$ change in temperature, there is approximately $1.5 \%$ change in the responsivity (number of excited electrons per absorbed photons) of the sensor.
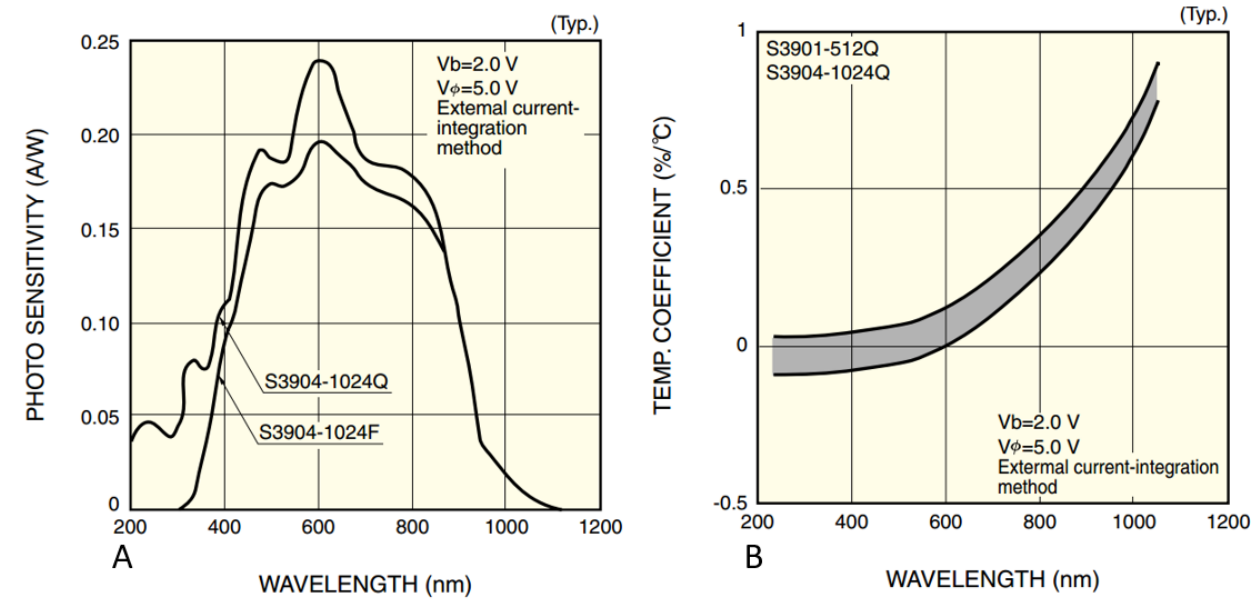

Figure 4.3. Hamamatsu Specifications - A) Spectral Responsivity of a typical u/vis chip from Hamamatsu. B) Thermal correction for responsivity as a function of temperature. Both figures are borrowed under fair use from Hamamatsu specification sheet ${ }^{90}$.

Referencing the Hamamatsu specification sheet, ${ }^{90}$ a $5{ }^{\circ} \mathrm{C}$ increase in temperature doubles the dark current in their low noise sensors, including the sensor found in the 
MMS-1 VIS/NIR spectrometer. Section 4.8 quantifies how a spectrum collection protocol for a shadow probe system using the MMS-1 is impacted by theoretically doubling the background noise, as well as a $1.5 \%$ change in the sensitivity of the detector.

\subsection{Two Methods for White Tile Reference}

Currently, commercial handheld spectrometers have two approaches, shown in Figure 4.4, for collecting the internal reference spectra. The Scio and the Sunforest use an external white reference target, which is scanned as part of the power-up process. The F-750 employs a white reference shutter, which can be scanned on demand, including during each measurement. Each approach has inherent advantageous and drawbacks. For example, the sole use of a power-up correction reduces the scan time for each measurement, but the one time correction can fail to correct when environmental conditions change. The sole use of a unique correction collected during each measurement can account for dynamic environmental conditions, but increases the scan time and can introduce a variable amount of instrument noise into each scan. 


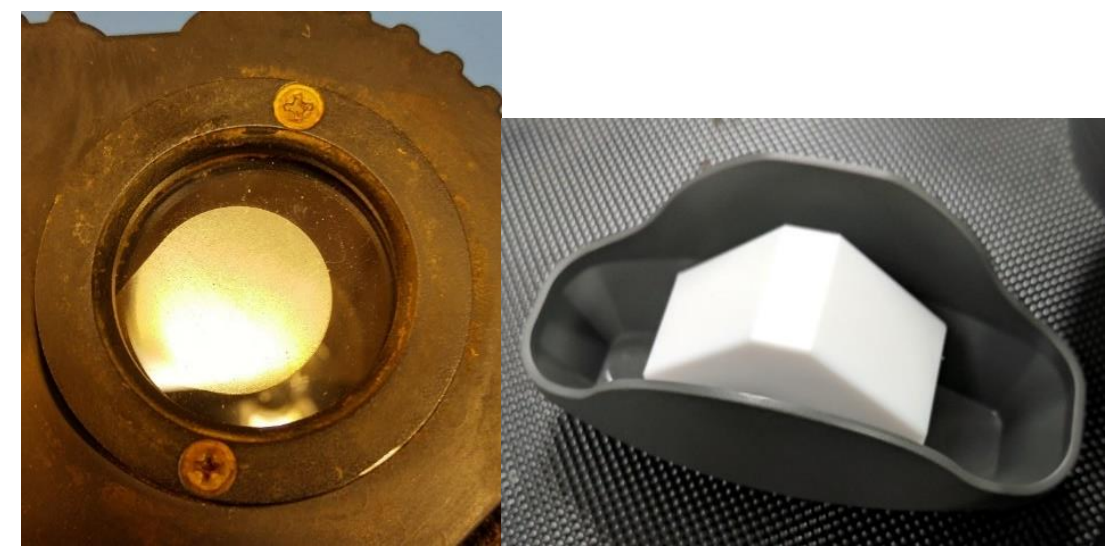

Figure 4.4. Two ways of White References are used Left) white reference shutter used in shadow probe instruments such as the F-750 Right) external white reference used by the Sunforest $\mathrm{H}-100$.

4.6 Comparison of Thompson grape calibration for an Internal on-demand vs External White Reference Target spectrometer system under Ideal Conditions

A single population of 40 Thompson grapes were scanned with a shadow probe spectrometer and SCiO (Figure 4.5). PLSR model statistics for each device is presented in table 4.1. The sugar content of the grapes was then measured to the nearest tenth of a degree Brix. Due to limitations in the SCiO Lab software, duplicate reference values prevented the system from preforming a regression. To counter this limitation, an extra digit of artificial precision was added to duplicate reference values, (If two grapes measured as $12.0 \%$ sugar content, then they were entered into the SCiO Lab software as 12.00 and 12.01). The extra digit of precision was not included in the analysis of the shadow probe data. 


\begin{tabular}{|c|c|c|c|c|c|c|c|}
\hline & \# PC & Wavelength $\mathrm{nm}$ & $\mathrm{R}^{2}$ & $\mathrm{R}_{\mathrm{cv}}{ }^{2}$ & RMSE & RMSECV & $\mathrm{N}$ \\
\hline $\begin{array}{c}\text { Shadow } \\
\text { Probe }\end{array}$ & 3 & $729-975$ & 0.91 & 0.89 & 1.08 & 1.29 & 40 \\
\hline SCiO & 5 & $759-1052$ & 0.897 & - & 1.158 & - & 40 \\
\hline
\end{tabular}

Table 4.1. Contrasts PLSR statistics for two shortwave-NIR handheld spectrometers.

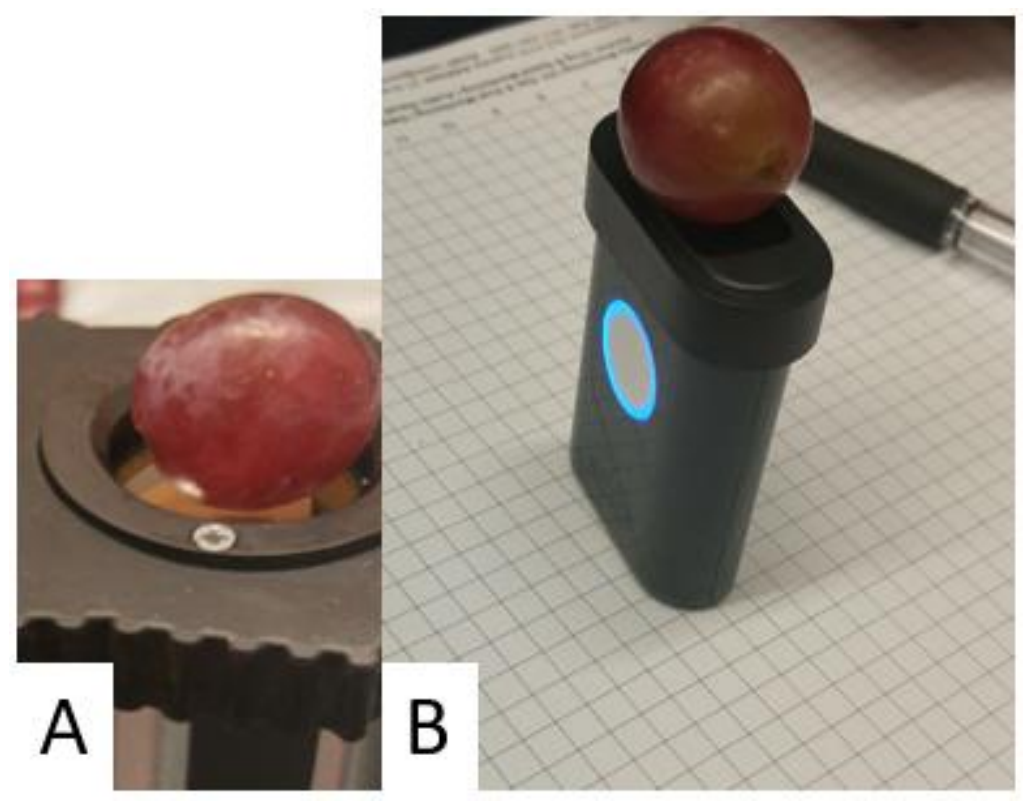

Figure 4.5. Sample presentation of a Red Thompson grape A) Shadow Probe B) SCiO

When external temperature and lighting are controled, the SCiO has comparable performance for table grapes to that of other spectrometer systems designed for fresh produce. This adequate performance of the SCiO under controlled environmental conditions is confirmed by Harpreet. ${ }^{91}$ The Thompson Grape experiment shows that 
neither the on-demand or at-power-up white reference are inherently adverse for data collection.

4.7 External White Reference Target under Ideal and Non-ideal Conditions

A traditional method of qualifying an instrument is to determine the repeatability of sample collection. To accomplish this, 20 repeated scans of a PTFE target were collected. The reflectance data were then captured from the browser and manually exported to Excel. As shown in Figure 4.6, even for pixels that receive little illumination (SCiO Illumination profile shown in Appendix-2) from the $750 \mathrm{~nm}$ LED source there is still below 1 milli-absorbance unit. A teardown of the SCiO Spectrometer can be found online at https://learn.sparkfun.com/tutorials/scio-pocket-molecular-scannerteardown. 


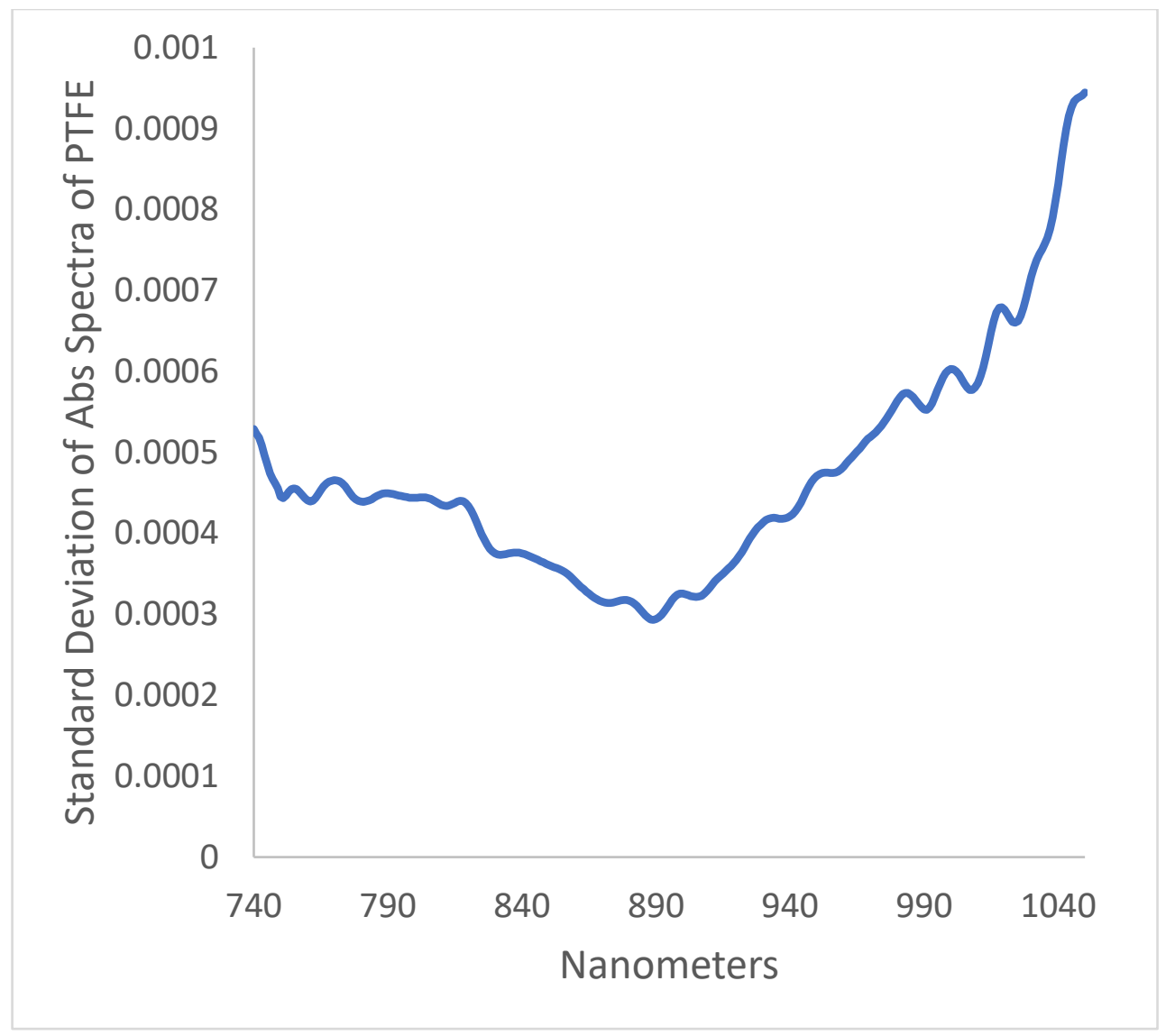

Figure 4.6. Standard deviation for 20 scans of PTFE as collected by the SCiO.

To test if the SCiO spectrometer has a susceptibility to temperature fluctuations, repeated scans of a PTFE target were collected at room temperature. Then the SCiO spectrometer was heated with a blow dryer until it was uncomfortable to touch. Repeated spectra of a thermally stable PTFE block were again collected as the instrument was allowed to cool. For both sets of scans, the SCiO spectrometer was in contact with the PTFE target. As shown in Figure 4.7, there is a shift in spectral intensity, but not wavelength assignment due to the thermal drift. Wavelength stability is 
important for issues such as model transfer and general instrument usability.

Additionally, the variability of the thermally dynamic case (yellow) appears to be larger than that of the blue thermally stable case around $725 \mathrm{~nm}$ for a thermally stable PFTE target. The raw data for Figure 4.7 were not extracted from the web platform for analysis.

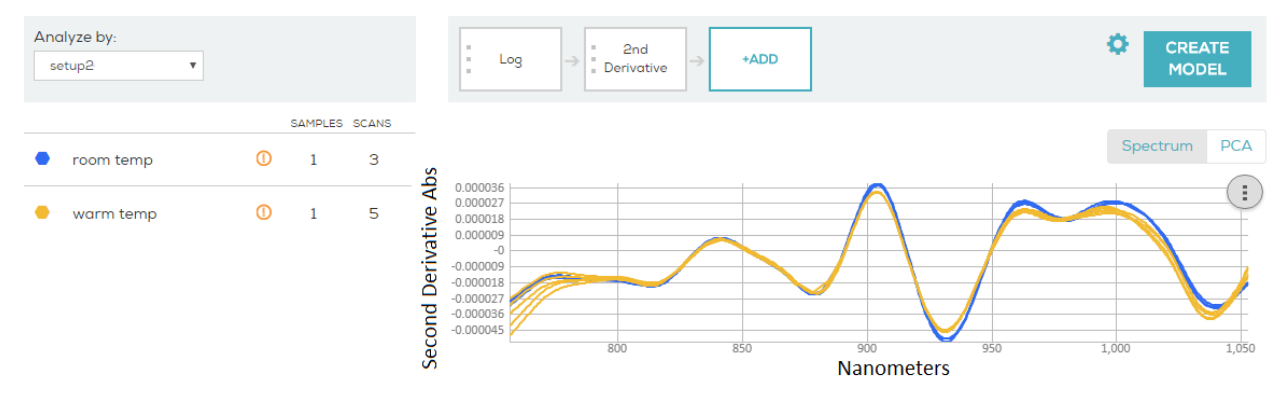

Figure 4.7. Influence of temperature on the spectra collected by the SCiO spectrometer. Note the increased spread of the thermally dynamic scans shown in yellow compared to the stable room temperature scans in blue.

4.8 Internal White Reference Target under Ideal and Non-ideal Conditions

An on-demand spectrum collection protocol used with the MMS-1 spectrometer is impacted by the theoretically doubling the background noise, as well as a $1.5 \%$ change in the sensitivity of the detector, can be quantified by analyzing repeated scans of a thermally isolated PTFE. To minimize the effect of radiative heat from the lamp, scans were collected every 5 minute under two conditions; a thermally stable and thermally dynamic. For the thermally dynamic case, the internal temperature of the MMS-1 was first warmed to $\sim 30^{\circ} \mathrm{C}$ and then allowed to undergo radiative cooling during spectra 
collection. Figure 4.8 shows the temperature profile inside of the MMS-1 VIS/NIR spectrometer used in the F-750 Produce Quality Meter (Felix Instruments, USA). Each of the four scans in the spectrum collection protocol were average of four scans.

The F-750 scans an internal white reference target to normalize the collected sample spectra during the calculation of absorbance. Figure 4.8a shows the detector output measured at $799 \mathrm{~nm}$ for the white reference target for both temperature profiles. In the thermally stable case, the detector output for $799 \mathrm{~nm}$ is also stable; the higher the temperature, the lower the ADC count. For the thermally dynamic case, the detector output (responsivity) fluctuates by approximately $2-3 \%$, which is consistent with a 5 degree change in temperature of the detector. Nonetheless, the fluctuations in detector response do not follow the monotonous change in the detector temperature as seen in Figure 4.8b. If the detector temperature and response had followed the same trend, then the fluctuation in response could be rationalized as only being due to the predicted change in dark current. This conclusion is further supported by the stability of the dark current reference spectra presented in table 4.2 . 


\begin{tabular}{r|r|l|l|l}
\multicolumn{1}{c|}{$\begin{array}{l}\text { Average } \\
\text { Dark } \\
\text { Current } \\
\text { Pixel } \\
\text { Value }\end{array}$} & $\begin{array}{l}\text { Maximum } \\
\text { Dark } \\
\text { Current } \\
\text { Pixel } \\
\text { Value }\end{array}$ & $\begin{array}{l}\text { Minimum } \\
\text { Dark } \\
\text { Current } \\
\text { Pixel } \\
\text { Value }\end{array}$ & $\begin{array}{l}\text { Standard } \\
\text { Deviation } \\
\text { of Dark } \\
\text { Current }\end{array}$ \\
\cline { 2 - 5 } $\begin{array}{r}\text { 2 Scans to Average } \\
\text { Thermally Stable }\end{array}$ & 2322.73 & 2360.35 & 2300.27 & 54.29 \\
\hline $\begin{array}{r}\text { 4 Scans to Average } \\
\text { Thermally Stable }\end{array}$ & 2279.19 & 2324.40 & 2259.23 & 26.31 \\
\hline $\begin{array}{r}\text { 2 Scans to Average } \\
\text { Thermally Dynamic }\end{array}$ & 2318.84 & 2354.52 & 2289.47 & 89.54 \\
\hline $\begin{array}{r}\text { 4 Scans to Average } \\
\text { Thermally Dynamic }\end{array}$ & 2373.03 & 2410.70 & 2347.63 & 86.70
\end{tabular}

Table 4.2. Comparison of pixel values for dark current correction in the F-750. 

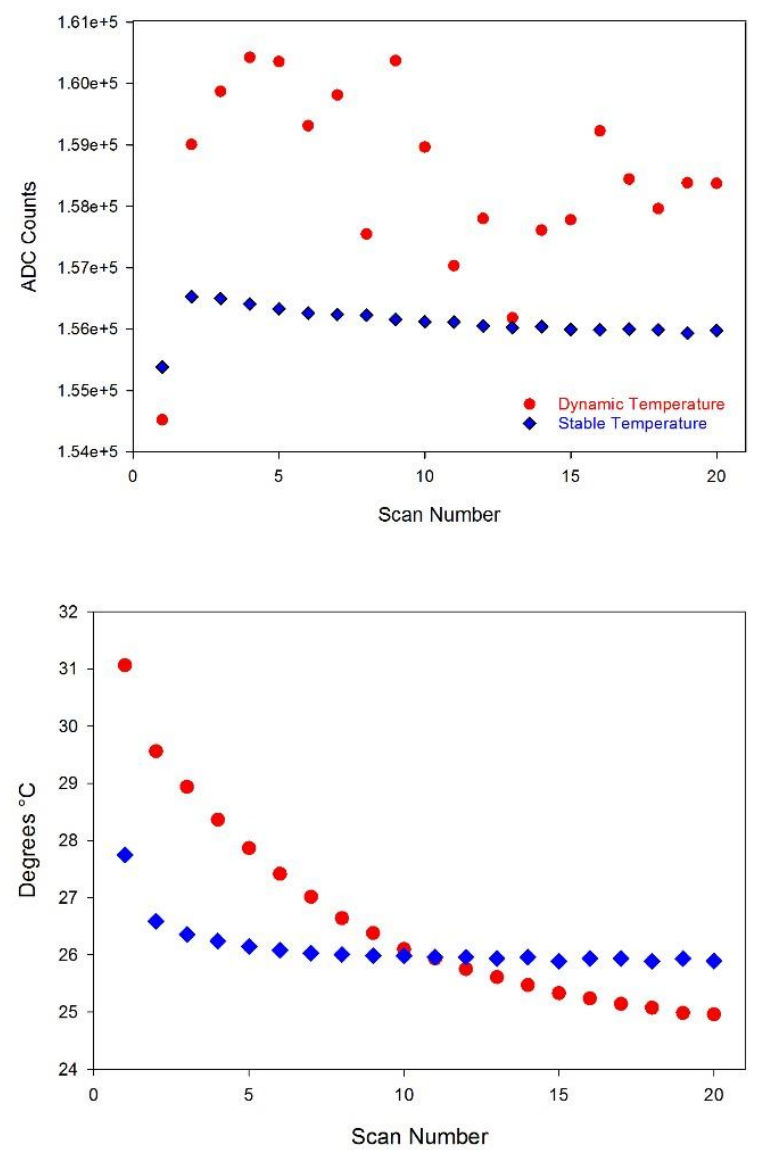

Figure 4.8. Thermal Influence on Stability of Spectra TOP) ADC counts or a digital signal read from the F-750 spectrometer for pixel $799 \mathrm{~nm}$ of the internal white reference for 20 repeated scans under stable and dynamic detector temperature conditions. BOTTOM) Temperature inside of MMS-1 housing at the beginning of each scan for the stable and dynamic conditions. 5 minute of lamp off delay between each scan. Environmental temperature was $\sim 21^{\circ} \mathrm{C}$.

To explain the variation in the detector output, we need to consider the fact that each spectrum was collected under dynamic conditions, whereas the specifications data, presented Figure 4.3, were collected under stable conditions at multiple temperatures. The detector temperature in the dynamic case is constantly changing, either throughout the 200 ms acquisition time or between individual measurements. This, in turn, adds an unquantified variable in the spectrum collection protocol, and therefore an increase 
in noise and hence the increase in standard deviation of the recorded spectra for a thermally isolated Teflon block shown in Figure 4.9.

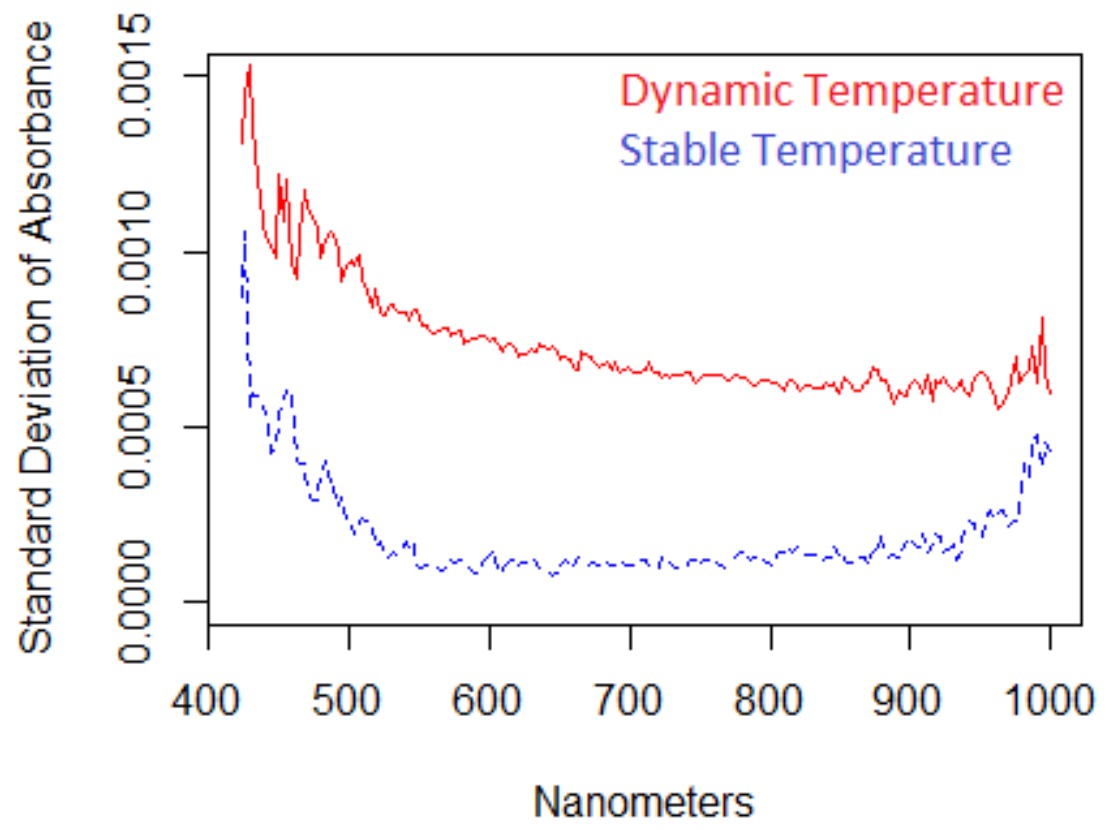

Figure 4.9. Standard Deviation of 20 repeated scans of an external thermally stable PTFE block collected under stable and dynamic in device temperature conditions with 4 scans to average for each individual spectrum used in the calculation. The Teflon spectra were calculated with a unique white reference scan for each collection event.

\subsection{The Role of Stray Light Corrections}

\subsubsection{SCiO Spectrometer Susceptibility to External Light}

To test if the SCiO spectrometer has a susceptibility to stray light, repeated scans of a PTFE target were collected with and without side lighting. The SciO spectrometer was in contact with the PTFE target. The side lighting was a $12 \mathrm{~W}$ tungsten lamp positioned $\sim 4$ 
inches from the PTFE target at 90 degree orientation to the detector. Repeated scans were again collected. As shown in Figure 4.10, there is a shift in spectral intensity, but not wavelength assignment.

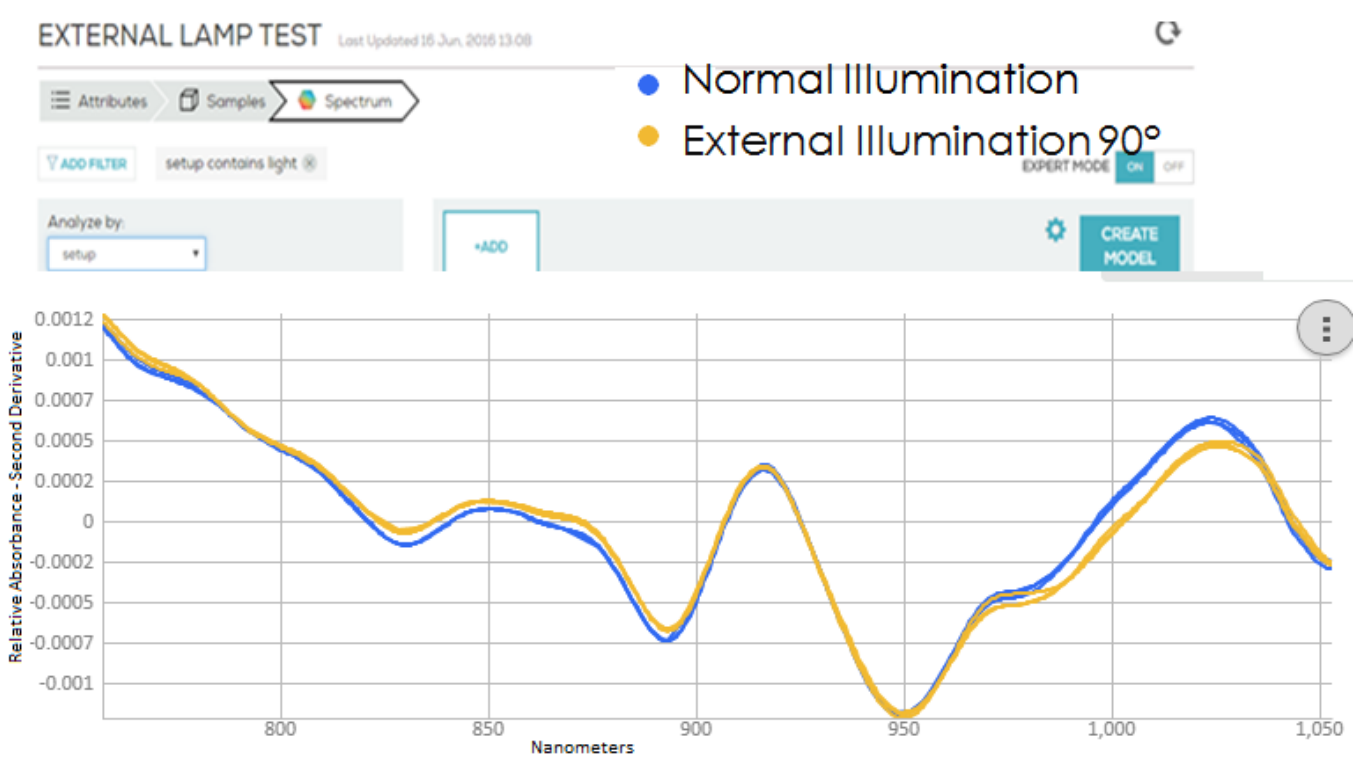

Figure 4.10. Influence of Stray light on the second derivative spectra collected by the SciO Spectrometer. The apparent red shift between the regular lighting shown in blue and the side lighting shown in yellow at $1025 \mathrm{~nm}$ is likely due to the additional infrared signal from the external lamp.

Minimizing the influence of external lighting is a necessity for field measurements.

When the SciO spectrometer was taken outside to measure cherry tomatoes on the bush under direct sunlight, the result was an error message. This is likely because the sunlight illuminating from within the fruit made the tissue appear brighter than or nearly as bright as the one-time white reference scan. 
By accessing information found in Google Chrome's developer tools (as available in June 2016), it was discovered that SciO uses a one-time dark and white reference spectrum to normalize the reflectance spectra. SciO's spectral collection protocol, as exposed in the code, does not directly mention the collection of a spectrum of the sample with the lamp off. As discussed in step 4 of section 4.1, a lamp-off sample scan can be used to normalized the calculated reflectance spectrum for ambient light and self-illumination of the sample. The error message in figure 4.11 could likely be avoided if a lamp-off sample scan was added to the spectral collection protocol. Evidence for this assertion is presented in section 4.9.2. 


\subsubsection{Susceptibility of the F-750 to External Light}

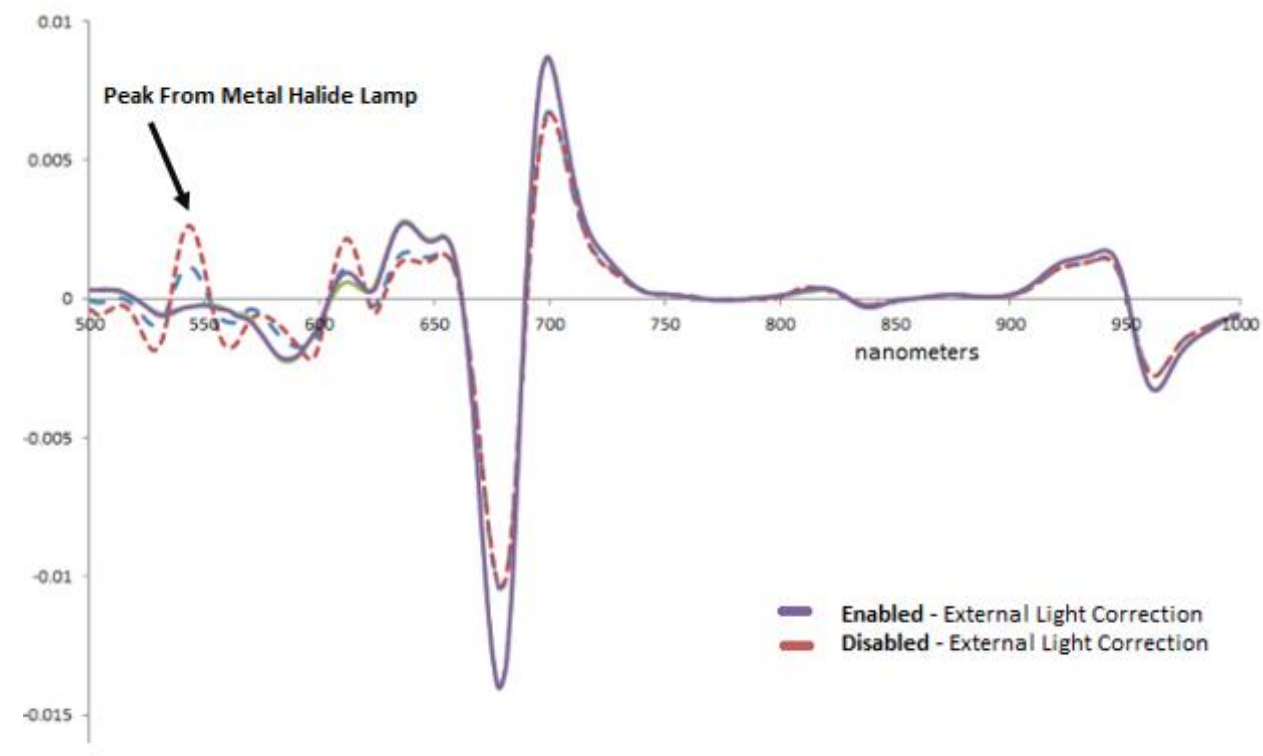

Figure 4.11. Spectra of a Thompson table grape under external lighting. The red dashed light is spectra collected without the lamp off shutter open correction for ambient light. The blue solid line is the lamp off shutter open correction for ambient light enabled.

The F-750 employs a built-in reference shutter to account for external light that penetrates through or around the fruit during the scan. By collecting spectra of the sample with the shutter open and the lamp off, the spectrometer is able to measure the self-illumination of the sample. To verify the correction routine is properly accounting for external light, a table grape was centered on the lens of the F-750 and spectra were collected with the correction routine enabled and disabled. As shown in the red dashed line of Figure 4.11, when this correction is disabled there is a peak at $~ 550 \mathrm{~nm}$ due to the Metal Halide ceiling lights. When the correction is enabled, the $550 \mathrm{~nm}$ peak is not apparent. Additionally, Dry Matter or Brix (sugar content) calibrations use the spectra 
window of 729-975 nm. The apparent change in spectral intensity is small between $729-$ $975 \mathrm{~nm}$ when the external light correction is enabled or disabled. However, these pixels are heavily weighted in a PLSR calibration for Dry Matter and the difference in spectra, with and without external light correction, is sufficient to cause a bias in prediction (data not shown).

\subsection{Conclusion}

The repeatability of spectra collected is directly linked to the accuracy of any predictions made from the data. If portable handheld spectrometers are to be taken into the field to collect data, which can inform farm managers' decisions, then it is important to account for how environmental factors influence the hardware's ability to collect reproducible of spectra. Moreover, if reliable field data are to be collected with handheld spectrometers, the spectrum collection protocols must normalize the calculated absorbance for the natural self-illumination of the sample. Only with these considerations can spectroscopy be used to increase the value of a farmer's crop enables the farmer to harvest fruit at the ideal maturity.

Portions of this chapter were previously published under: Lerud, R. (2015). Demystifying the Black Box: Improving Detection Limits. NIR News, 26(3), 14-15. https://doi.org/10.1255/nirn.1523

Lerud, R. (2016). An Experiment on the Effects of Detector Design and Thermal Properties as a Function of Noise. NIR News, 27(8), 25-27. https://doi.org/10.1255/nirn.1652 


\section{Chapter 5}

Comparison of Derivative Quotient Method and PLSR for SWNIRS model development and transfer 


\subsection{Evaluation of the Derivative Quotient Method For Spectral Regression}

The following work explores the derivative quotient method (DQM) as an alternative to partial least squares (PLSR) for tree fruit spectra. As such, various experiments and boundary conditions for the DQM routine are presented. The sugar water dataset from chapter 3 was initially selected to provide context for the gap and smoothing derivatives used by the DQM routine in contrast to the presented previously results with SavitzkyGolay filters. Subsequentially, a more robust sugar water dataset was developed to explore the potential DQM for calibration transfer between portable handheld spectrometers. Given the relative success of the sugar water experiments, a large calibration transfer exercise was conducted with field data from 6 portable spectrometers. This chapter demonstrates the potential of DQM for real world applications.

Prior to the advent of personal computers, near infrared (NIR) spectra were correlated to analyte levels using Multiple Linear Regression (MLR). Partial least squares regression (PLSR) was developed by $1966^{34}$, and with the development of the personal computer, PLSR soon came to dominate NIR chemometrics. In the 1970 s Karl Norris ${ }^{36,92}$ developed the quotient derivative regression method or DQM (cited several times in literature as the Norris Regression ${ }^{93-96}$ ). NIRSystems (now part of FOSS Gmbh, Denmark) incorporated MLR using derivative terms into their NSAS software ${ }^{97}$ and included a "Norris Regression" that optimized the gap for a given segment in MLR regressions. The 
US Department of Agriculture incorporated DQM into their SPAN software 98 35, 92, 99. There is one published comparison of DQM and PLSR in terms of model performance ${ }^{100}$ and none on the transferability of models between instruments.

Norris and Hruschka defined a gap first derivative ${ }^{9}$ according to equation 13 . All higher derivatives are calculated by sequential applications of equation 13 , with the same gap. In addition, a "box-car" smooth may be applied to the derivative (or to the original spectrum, the order of differentiation and smoothing does not matter to the final result). The smooth gap (smt) variable is defined so that the number of points in the smoothing interval is always an odd number (equation 14). The integer coefficients of the second derivative convolution function (CF) always show the $1,-2,1$ pattern, the number of zero separating the -2 with the 1 's given by $2 \times$ gap -1 . The CF for the combination of the derivative and smooth is given by a process similar to multiplication of the individual CFs, and the overall normalization constant is the product of the individual normalization constants.

$$
\frac{d y}{d x}=\frac{f(x+g a p)-f(x-g a p)}{2 \times g a p}
$$

where $f(x)$ is each successive $y$ value in the original absorbance spectrum, and gap is any number (greater than 0 ) of intervals along the $x$ axis. 


$$
N=2 \times s m t+1
$$

where $N$ is the number of $y$ values averaged for the smoothed result, and smt may be 0 (no smoothing) or any number of $x$ axis intervals.

DQM routines were recently implemented in MATLAB by David Hopkins ${ }^{101}$. The routine DQM1 is an iterative search for quotient terms of wavelengths which minimizes the error (SEC) in an MLR. DQM1 accepts user input for the wavelength range, the number of quotient terms, an upper limit on the size of the derivative gap, smooth gap, the order of the derivative and the range of search wavelengths for the numerator and denominator. Starting with the first term of the calibration, wavelengths are initially selected from Gap-Smoothing derivatives ${ }^{6}$ with a smoothing of 0 . Once the "best" ratios of Gap are selected, a smoothing routine inspects the standard error of calibration (SEC) of the calibration and systematically increasing the smoothing value, and selects the wavelengths for the numerator and denominator terms which give the lowest SEC. Then the process is repeated for the next term or terms of the calibration, if desired.

For each value in a spectrum, the Gap-Smooth derivative calculation involves the use of a convolution function (CF) which is multiplied by the respective absorbance values as a sliding window passed over each wavelength value in a spectrum. For example, a second derivative with a gap of 4 and smt of 4 has a CF of $\left[\begin{array}{lllllllllllllllll}1 & 1 & 1 & 1 & 1 & 1 & 1 & -1 & -2 & -2 & -2 & -2 & -2 & -2 & -1\end{array}\right.$ 11111111 ] /576. It should be noted that this CF has no equivalent in segment-gap nomenclature. Another second derivative with a gap of 2 and smt of 1 has a 11-point CF 


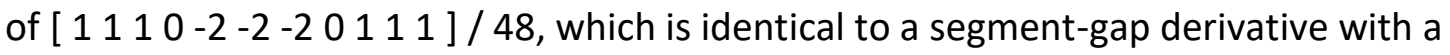
gap of 1 and segment of 3 . One more example is a second derivative with a gap of 2 and smt of 0 , which has a 9-point CF of $\left[\begin{array}{llllllllll}1 & 0 & 0 & 0 & -2 & 0 & 0 & 0 & 1\end{array}\right] / 16$, which is identical to a segment-gap derivative with a gap of 3 and segment of 1 . Segment-gap derivatives are used in The Unscrambler and were available in NSAS and Vision software. To add to the complexity, it should be noted that "gap" can be defined as twice the interval used here ${ }^{101}$, so the user must be careful to observe the usage in a particular paper or software package. Smoothing of the random noise is a result of the whole CF.

DQM models can be compared directly to PLS models for a given population set and calibration task. Another test of functionality lies in the robustness of DQM and PLS models across instruments.

5.2 Understanding how the Gap-Smoothing Convolution Function is used by DQM Initially, DQM was evaluated with the sugar water dataset collected in section 3.3. This data was used as a proof of concept beta test of the DQM1.m program. Table 5.2 below contrast the wavelengths, gap and smoothing parameters selected by the DQM1.m program for the first and second derivative calibrations. The DQM calibrations uses quotient or ratio terms of the center wavelengths listed in Table 5.1. 
Table 5.1. DQM calibration wavelengths for sugar water data from section 3.3.

\section{Calibration Center Wavelength}

\begin{tabular}{|l|l|}
\hline $\mathbf{1}^{\text {st }}$ Derivative & (1st Derivative $981 \mathrm{~nm}$, gap=5, smt=0) \\
DQM Term \#1 & (1st Derivative $903 \mathrm{~nm}$, gap=5, smt=1) \\
\hline $\begin{array}{l}\mathbf{1}^{\text {st }} \text { Derivative } \\
\text { DQM Term \#2 }\end{array}$ & $\begin{array}{l}\text { (1st Derivative } 819 \mathrm{~nm}, \text { gap=2, smt=0) } \\
\text { (1st Derivative } 981 \mathrm{~nm}, \text { gap=1, smt=0) }\end{array}$ \\
\hline $\begin{array}{l}\text { 2nd Derivative } \\
\text { DQM Term \#1 }\end{array}$ & $\begin{array}{l}\text { (2nd Derivative } 951 \mathrm{~nm}, \text { gap }=5, \mathrm{smt}=0) \\
\text { (2nd Derivative } 750 \mathrm{~nm}, \text { gap }=4, \mathrm{smt}=1)\end{array}$ \\
\hline $\begin{array}{l}\text { 2nd Derivative } \\
\text { DQM Term \#2 }\end{array}$ & $\begin{array}{l}\text { (2nd Derivative } 984 \mathrm{~nm}, \text { gap }=2, \mathrm{smt}=0) \\
\text { (2nd Derivative } 831 \mathrm{~nm}, \text { gap }=6, \mathrm{smt}=3)\end{array}$ \\
\hline
\end{tabular}

The Figure 5.1 contrasts the four preprocessed spectra used in the DQM regression and the single preprocessed used in PLS. The input absorbance spectra were identical for all 5 plots. A discussion of the bandwidth of a computer generated Gaussian peak, size of the gap, and the quality of the resulting derivative can be found in Ritchie $^{94}$. In the case of short wave near infrared spectra, the bandwidth of the spectral peaks is sufficiently large ( 20nm) for the gap and smoothing calculation of derivative spectra. 


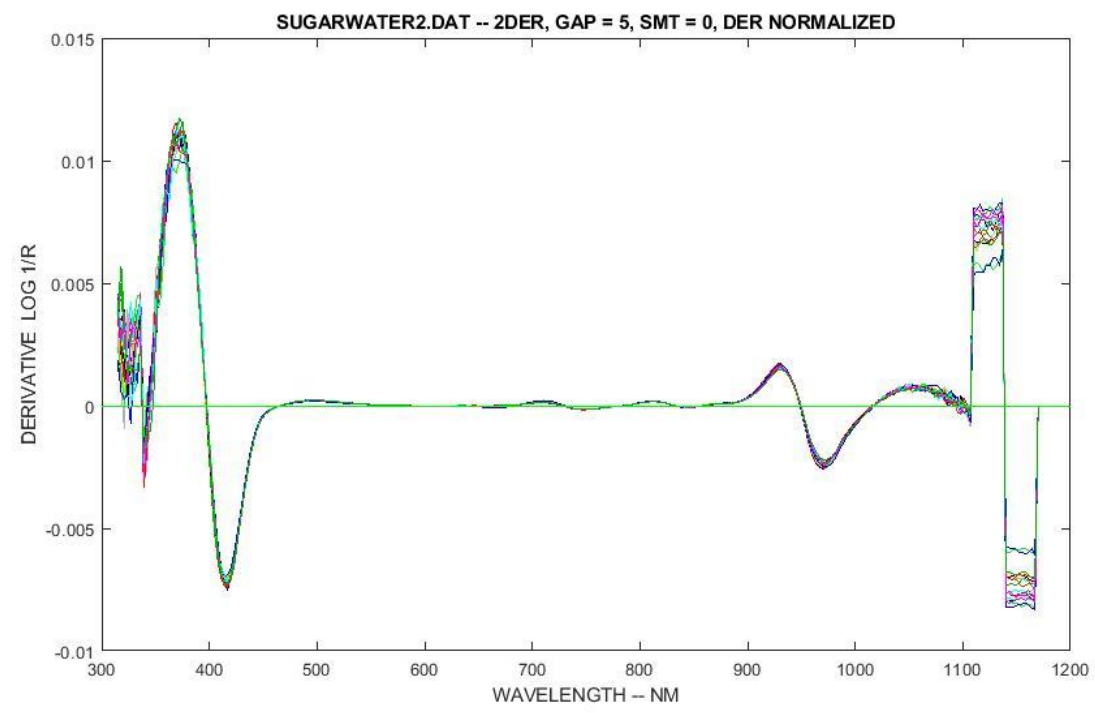

Figure 5.1. First term numerator is selected from the second derivative calculated by Gap=5,Smooth=2 filter.

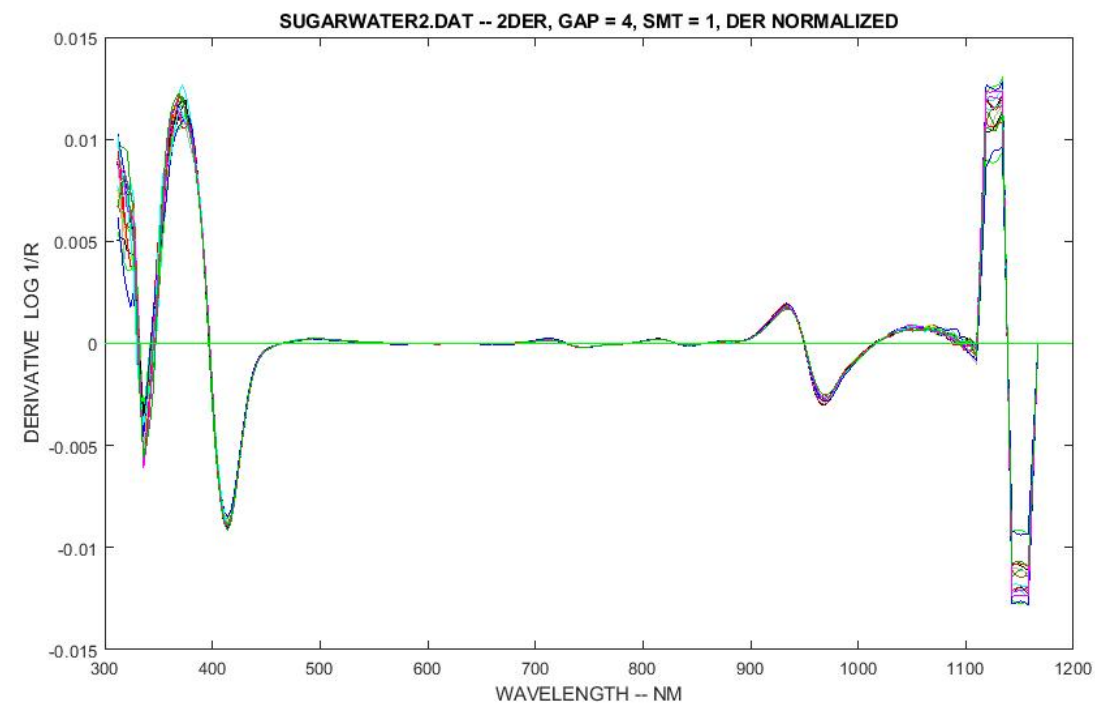

Figure 5.2. First term denominator is selected from the second derivative calculated by Gap=4, Smooth=6 filter. 


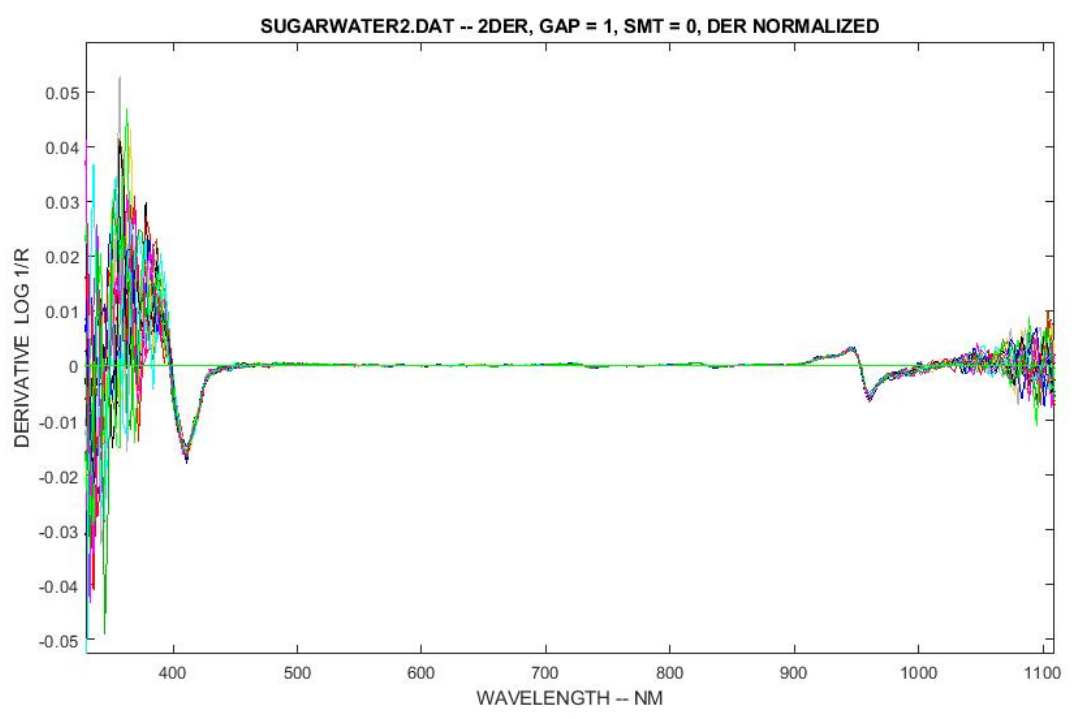

Figure 5.3. Second term numerator is selected from the second derivative calculated by Gap=1, Smooth=2 filter.

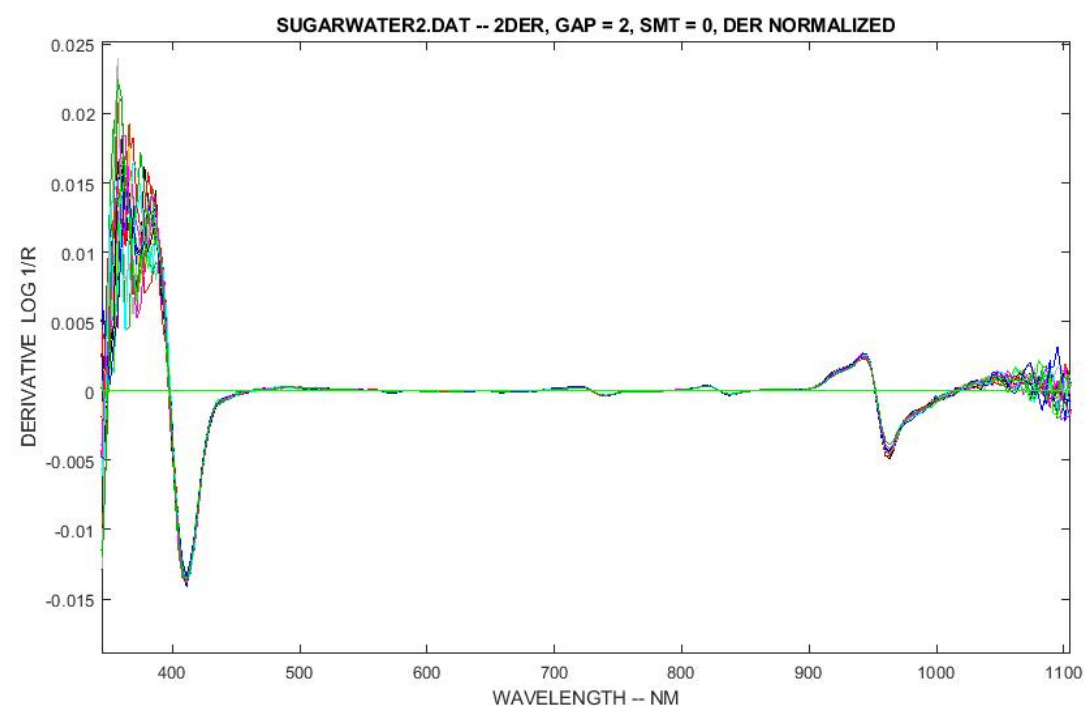

Figure 5.4. Second term denominator is selected from the second derivative calculated by Gap=2, Smooth=2 filter. 


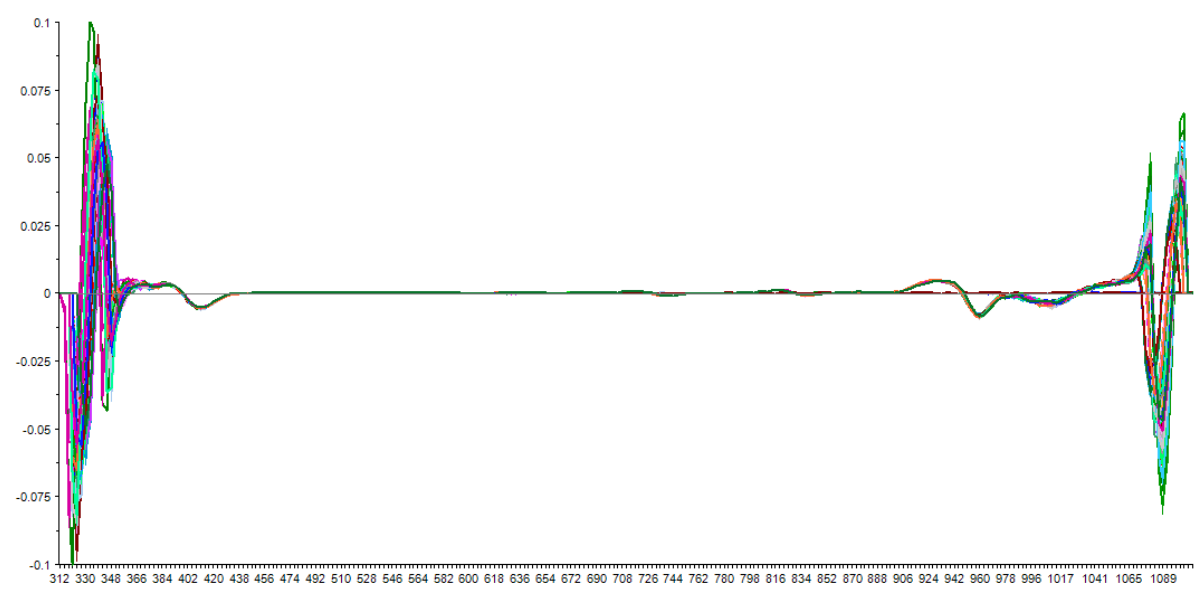

Figure 5.5. Second derivative spectra used by PLSR is calculated by 9 Point Second Order Savitzky-Golay filter.

As demonstrated in Figures 5.1-5.5 the Gap-Smoothing convolution function has the ability to change the overall shape of the second derivative spectra. For example, the shape and apparent noise around $1000 \mathrm{~nm}$ are drastically different between Figures 5.1 and 5.3. Whereas the spectra in Figure 5.5, used for PLSR, is limited to a single fixed convolution function and does not benefit from the ability to use a different smoothing functions for each wavelength band in the regression. Additionally, DQM uses wavelength ratios in regression whereas PLS uses an additive weighting for every pixel.

Looking at only the numerator terms in the second derivative DQM calibration, both 942 $\mathrm{nm}$ and $951 \mathrm{~nm}$ can be assigned to the $\mathrm{R}-\mathrm{OH}$ 3rd overtone. In addition to the center wavelength, $B$, it is important to consider all wavelengths operated on by the convolution function to generate the second derivative center wavelength. In the case of the first term numerator, the normalized convolution function, spans 10 wavelengths or pixels on each side of the center wavelength. Because the F-750 uses 3nm 
interpolated pixels, the convolution function is taking a weighted sum over $30 \mathrm{~nm}$ on each side of the center wavelength. From Figure 5.6 it can be seen that the first numerator term consists predominately of the $\mathrm{R}-\mathrm{OH}$ and water $3^{\text {rd }}$ overtone region (center wavelength $951 \mathrm{~nm}$, segment wavelength 981, $921 \mathrm{~nm}$ ). Whereas the numerator from the second term has a much shorter convolution function weighing 882,894 and $906 \mathrm{~nm}$. The denominator for both terms follow a similar format, the first term uses a rather large averaging window and the second term a smaller window. The first term denominator takes the weighted average of the $4^{\text {th }}$ overtone water peak ${ }^{102}$ (723 nm, $726 \mathrm{~nm}, 729 \mathrm{~nm})$, an R-CHx overtone peaks at (747 nm, $750 \mathrm{~nm}, 753 \mathrm{~nm}$ ) and (771 nm, $774 \mathrm{~nm}, 777 \mathrm{~nm}$ ). The second denominator term is taking the weighted averages $831,798,867 \mathrm{~nm}$. 


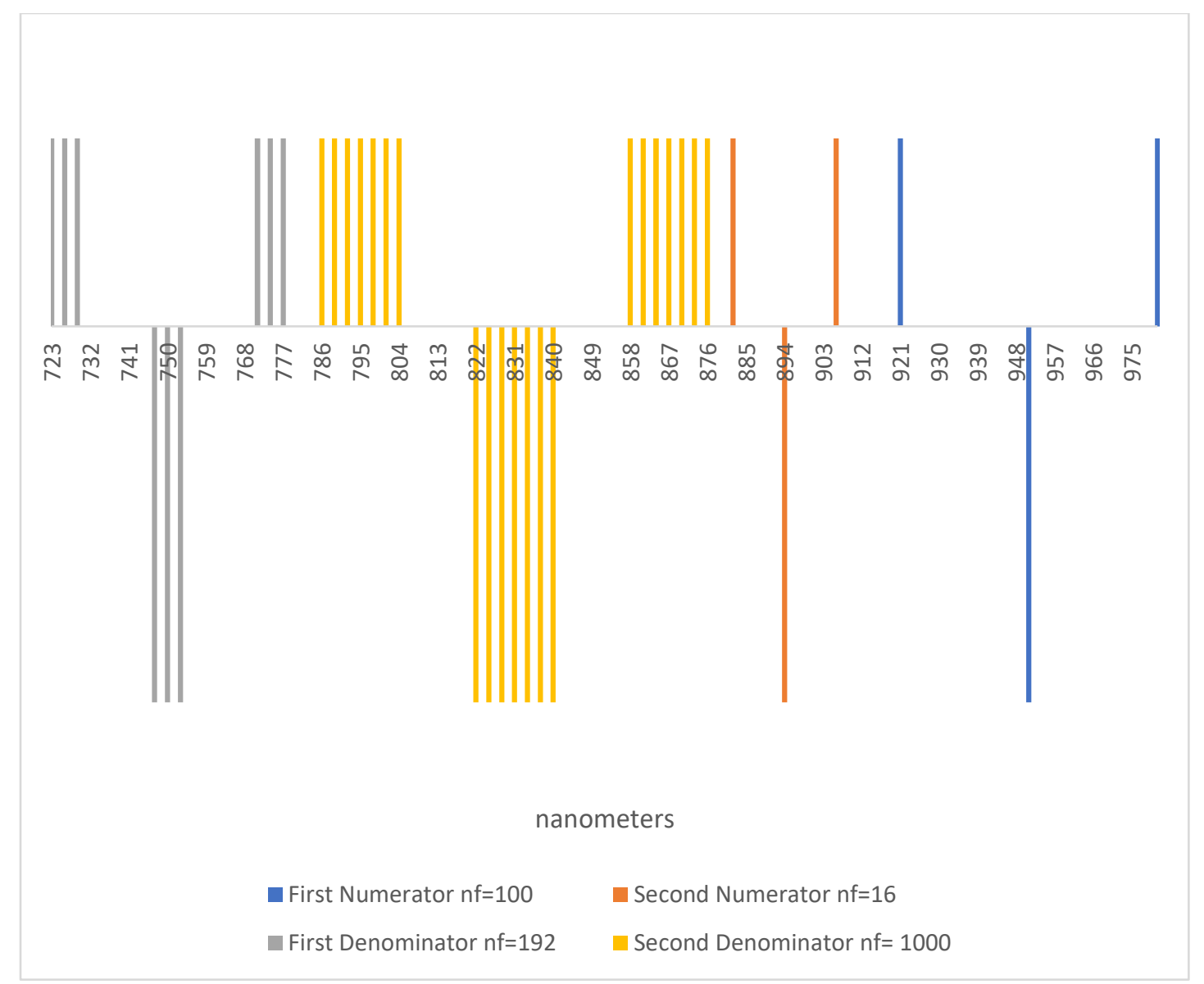

Figure 5.6. The convolution function for each for the numerator and denominator terms of the second derivative DQM sugar water calibration. 


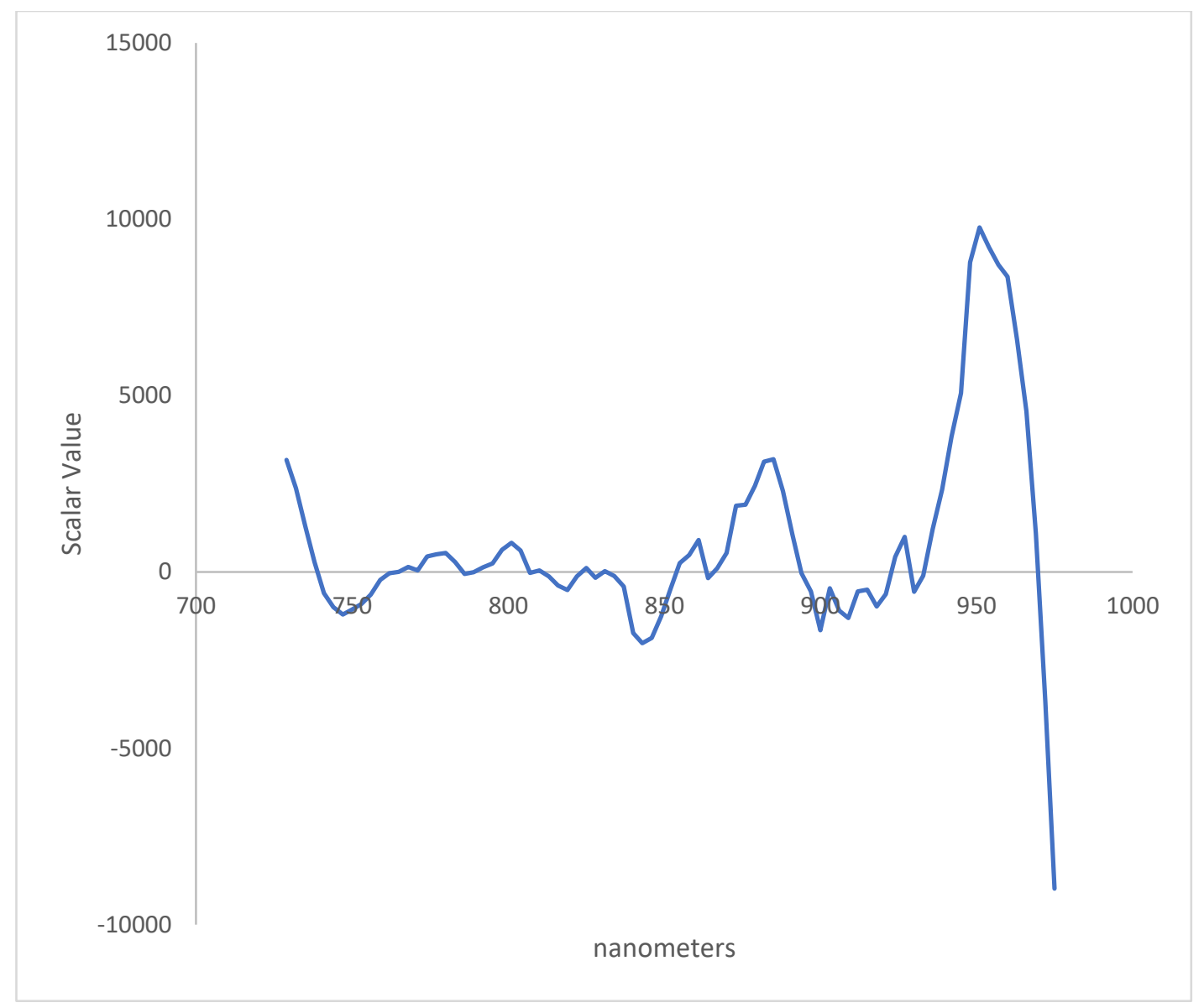

Figure 5.7. PLS Regression Vector for Sugar Water.

When attempting to understand what the DQM calibration is physically calibrating against, it is important to consider all of the absorbance wavelengths used to calculate the derivative wavelengths used in the regression. By looking at all of the absorbance wavelengths in Figure 5.6, it becomes clear that the calibration is actually dependent on 17 different wavelengths, which correspond to the chemical bonds that make up the sugar water mixture under investigation. Conversely, The PLS regression vector shown in Figure 5.7 spans 82 wavelengths. The PLS regression vector in Figure 5.3 also indicates 
that the sugar water calibration is heavily dependent on the $\mathrm{R}-\mathrm{OH}$ and water overtone peaks located at $940 \mathrm{~nm}$ and $960 \mathrm{~nm}$.

The DQM3 routine has a function which plots the correlation between a wavelength with a specific gap and smoothing derivative and reference value. Figure 5.8 shows that the numerator of term 1 is highly correlated with the sugar water concentration. Whereas, Figure 5.9 shows that the denominator of term 1 is not correlated with the sugar water concentration. This implies that the DQM routine is searching for wavelength pairs the first wavelength correlates with the reference value in question. The second wavelength acts as a reference or normalization for the regression.

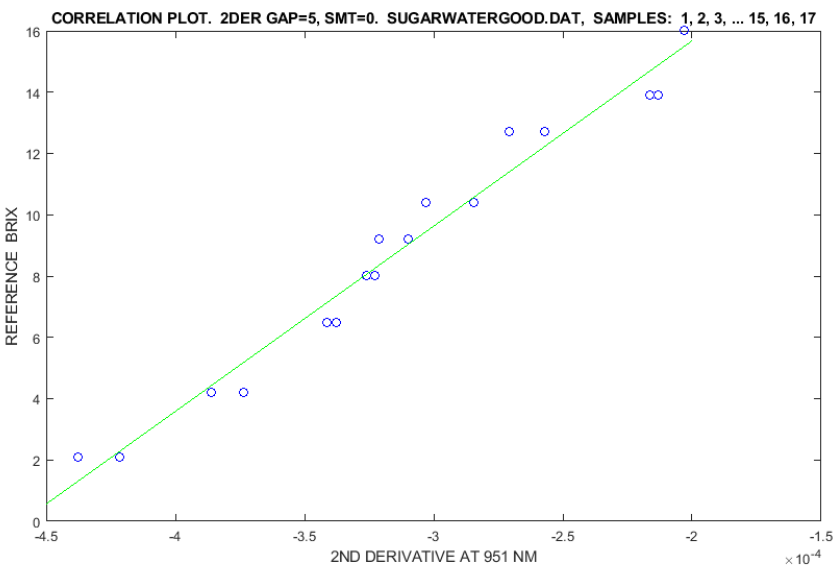

Figure 5.8. Correlation between first term numerator and sugar water. 


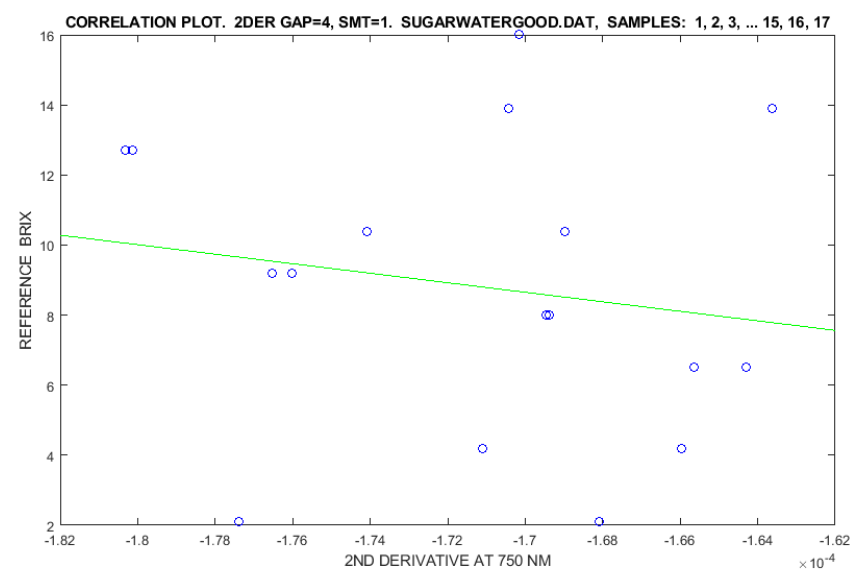

Figure 5.9. Correlation between first term denominator and sugar water.

\subsection{Aim of Study II}

The aim of this study was initially to beta test the DQM software with a non-wheat FTNIR dataset. Given the general success of the method, presented in 5.2, the study was expanded to include calibration transfer performance. Additionally, this study documents the largely ignored work of Karl Norris and presents an alternative to the increasing trend in recent literature towards neural networks and other Al methods.

To this aim, the following study compares transfer methods for the porting of models between instruments, expanding an earlier considerations ${ }^{103,104}$ to include Direct Transfer of DQM from master to slave and Global DQM calibrations. The work focused on the application of SWNIRS to internal quality of fruit, so this study was framed by work with a spectrometer system and data set relevant to that application. 


\subsubsection{Background on Model transfer}

Model transfer between instruments can be approached in three ways (Fearn ${ }^{105}$, Andrew and Fearn ${ }^{106}$, Soldado et al. ${ }^{107}$, Igne et al. ${ }^{108}$ ): (i) by making of a model that transfers without requiring standardization, e.g. using spectral pre-treatments, selecting wavelengths ranges that exhibit spectral 'stability', or by including data from multiple instruments in the calibration set; (ii) adjusting the model output such that it works on other instruments, e.g. simple slope and bias correction; (iii) correcting spectra from slave instruments to appear as they were acquired on master instrument, or (iv) transfer by orthogonal projection (TOP), with removal of spectral differences orthogonal to the calibration model.

The third approach in calibration transfer, of adjusting spectra from slave instruments to appear as master instrument spectra, has been addressed by a range of techniques. The spectral correction methods of spectral slope/bias correction (SSBC) and spectral bias correction ${ }^{109}$, involve wavelength by wavelength simple linear regressions of spectra from each instrument. Other techniques, as reviewed by Fearn ${ }^{105}$, include direct standardization (DS), piecewise direct standardization (PDS), double window piecewise direction standardization (DWPDS), orthogonal signal correction (OSC), finite impulse response (FIR) and wavelet transform (WT).

Other approaches achieve an 'implicit' orthogonalization, such as the 'repeatability' (REP) file concept ${ }^{110}$. In this method, difference spectra (of the same sample scanned on 
multiple instruments) are scaled proportional to the ratio of the number of samples in the REP file and calibration set, then assigned an attribute value of zero and included in the calibration set. In a similar approach, Saranwong and Kawano ${ }^{111}$ proposed a method for transfer of a partial least squares (PLS) calibration on apple total soluble solids (TSS) between two Foss NIR Systems 6500 spectrometers, in which the difference in the average spectra (second derivative absorbance) of a group of samples is used to adjust the slave instrument spectra by simple subtraction. This method affects a bias correction (difference spectrum multiplied by model coefficients) and cannot improve the SEP (bias corrected RMSEP) of the transferred model. In the case presented, the method was effective, with the resulting bias at the same level as the validation results on the master instrument.

\subsubsection{Equipment and software}

Spectra were acquired using F-750 Produce Quality Meters (Felix Instruments, Camas, U.S.A.). These units employ a Zeiss MMS1-NIR Enhanced (Carl Zeiss GmBbH, Jena, Germany) spectrometer in an interactance geometry employing a $5 \mathrm{~W}$ lamp with an inbuilt reflector (not parabolic) and a cylindrical barrel between lamp and sample. In this geometry a probe receiving light to the MMS1 fiber optic placed in front of a halogen lamp mounted in a parabolic reflector, such that a shadow is cast on the sample, and the detected light is largely derived from this shadowed area ${ }^{112}$. Additional details can also be found in Chapter 3. 
The current model of the MMS-1 units employs an S8381 Si PDA detector (Hamamatsu Photonics K.K., Hamamatsu City, Shizuoka, Japan). The original MMS-1 (part number 224001-9001-000; serial number 3017XX), employ an S4874 Si PDA detector (Hamamatsu Photonics K.K.). This original MMS-1 is employed by instrument in the following exercises. The change in the array used in the MMS-1 was made by Zeiss to reduce signal carryover between successive readouts and improve read-out time, but the array is less sensitive past $800 \mathrm{~nm}$.

PLSR models were developed using MATLAB R2014a (MathWorks Inc., Natick, MA, USA) with PLS toolbox 7.3 (Eigenvector Research Inc., Wenatchee, WA, USA). PLSR models were developed based on mean-centered second derivate absorbance spectra (Savitzky-Golay, second order, 9 points ${ }^{88}$ ). The wavelength range utilized was optimized in terms of RMSECV using a moving PLSR interval algorithm ${ }^{113}$, with the spectral window was iteratively varied to consider all possible ranges starting at $720 \mathrm{~nm}$ and ending at $1050 \mathrm{~nm}$, with a minimum window of $99 \mathrm{~nm}$.

DQM models were developed using the DQM program V2.3 written by David Hopkins ${ }^{100}$ in MATLAB R2014a. DQM models were developed using absorbance spectra based on a Gap and Smooth first or second derivative with center wavelengths in the $750-1020 \mathrm{~nm}$ wavelength range, using the DQM1 menu routine. DQM wavelength search was limited to the same range as used for PLSR, with the maximum gap and smooth interval set to 14 and 4, respectively, except as reported later. 
Model performance was assessed in terms of assessment of a test set (separate to the calibration set), using the statistics of validation $\mathrm{R}^{2}$, root mean square of errors of prediction (RMSEP) and bias-corrected RMSEP (SEP $)$.

5.3.4 Exercise 1 - Comparison of DQM and PLSR for sugar water mixtures Spectra of 19 sugar water solutions ranging from $0.0-13.2^{\circ} \mathrm{Brix}$, were acquired with each of three F-750 instruments, herby referred to as E, F, and G, with five replicate scans per sample. A set of 14 solutions were used for calibration $(n=70, \mu=6.1, \sigma=4.8$ ${ }^{\circ}$ Brix) and a set of five solutions for validation $\left(n=25, \mu=6.1, \sigma=3.8^{\circ}\right.$ Brix). These spectra were collected using a transflection geometry. This geometry involved placement of the F-750 probe onto one side of a cuvette (69 $\mathrm{mm}$ sided cube of $2.0 \mathrm{~mm}$ thick glass) with a $10 \mathrm{~mm}$ thick polytetrafluoroethylene (Teflon) block placed on the outer surface of the opposite side of the cuvette. Brix values were determined by a DR-103 Digital Refractometer (Bellingham and Stanley Ltd, Farnborough, U.K.) with a manufacturerrated $+/-0.2^{\circ}$ Brix uncertainty.

To control the temperature of the sugar water solutions, pre-made sugar water solutions were stored in a $25^{\circ} \mathrm{C}$ water bath, poured into the cuvette and spectra collected within 4 min. A thermocouple (on a digital multimeter, Klein Tools MM400, Klein Tools Inc., Lincolnshire, U.S.A.) confirmed that there was minimal temperature change during spectral acquisition $\left(<0.3^{\circ} \mathrm{C}\right.$ in any individual sample during spectra acquisition by the three spectrometers, and a range of $23.8-24.3^{\circ} \mathrm{C}$ across all 
measurements). PLSR models on each instrument were developed using 2 principal components using the wavelength range $816-918 \mathrm{~nm}$. The optimized calibration ranges are shown in Table 5.2.

The program DQM1, found that a second derivative two-term calibration best fit the sugar water calibration and validation data collected on the master instrument. DQM was able to produce equivalent calibrations using first derivative spectra (data not shown). The selection of center wavelengths, gap sizes, and smoothing windows, allows for the DQM calibration to span nearly the same wavelength range as the contiguous region spanned by PLSR. Using the calibration equation for instrument $F$ as an example, the numerator of Term 1 in equation 15 has a gap of 4 and a smoothing window of 4 , this results in the calculation of the second derivative spectrum by a convolution function which spans a $105 \mathrm{~nm}$ window of the absorbance spectrum.

Predicted Value $=\frac{(2 \mathrm{nd} \text { derivative } 873 \mathrm{~nm} \mathrm{gap}=4 \mathrm{smt}=4)}{(2 \mathrm{nd} \text { derivative } 879 \mathrm{~nm} \text { gap }=8 \mathrm{smt}=2)}+\frac{(2 \mathrm{nd} \text { derivative } 798 \mathrm{~nm} \text { gap }=2 \mathrm{smt}=1)}{(2 \mathrm{nd} \text { derivative } 807 \mathrm{~nm} \text { gap }=11 \mathrm{smt}=0)}$

Table 5.2. Wavelength range / parameters used for PLSR and DQM models for the sugar water data set, using Savitzky-Golay and gap smooth derivatives, respectively. DQM wavelengths are presented in the order of term 1 numerator, term 1 denominator, term 2 numerator, term 2 denominator, each with associated gap and smooth for the second derivative calculation.

\begin{tabular}{lll}
\hline Instrument & $\begin{array}{l}\text { PLS Wavelength } \\
\text { range }(\mathrm{nm})\end{array}$ & DQM wavelengths (gap, smooth) \\
\hline $\mathrm{X}$ & $807-912$ & $903(3,0), 852(12,0), 813(2,0), 807(6,0)$ \\
$\mathrm{Y}$ & $816-918$ & $873(4,4), 879(8,2), 798(2,1), 807(11,0)$ \\
$\mathrm{Z}$ & $753-912$ & $801(11,1), 846(12,0), 924(13,0), 885(3,0)$ \\
\hline
\end{tabular}




\subsubsection{Exercise 2 - Direct calibration transfer of sugar water models}

The effectiveness of DQM relative to PLSR for direct calibration transfer between instruments was tested using the sugar water validation data collected in Exercise 1 . To understand the potential of DQM for direct calibration transfer between instruments, the sugar water validation data collected on two slave instruments was predicted using the master calibration. The instrument $X$, has a wavelength assignment error of approximately $3 \mathrm{~nm}$ which in the case of sugar water dataset prevented PLS from adequately predicting the data. As shown by table 5.3 both PLSR and DQM gave approximately equivalent calibration and transfer results for nearly every combination of master and slave. There were no statistically significant differences between the SEP of each group as determined by one-way ANOVA $(F(2,15)=0.03, p=.05)$ (Table 5.3). 
Table 5.3. Validation statistics for sugar water (Brix) models used in prediction of an independent test set on several instruments. Exercise 1 (single instrument) results are shown in italicized bold, exercise 2 (model transfer between instruments) results in normal text.

\begin{tabular}{|c|c|c|c|c|}
\hline Instrument & Model & Bias & SEP & $R_{\text {val }}{ }^{2}$ \\
\hline \multicolumn{5}{|l|}{$\mathrm{Y}$ as master } \\
\hline$Y$ & PLSR & 0.02 & 0.17 & 1.000 \\
\hline$Y$ & $D Q M$ & -0.10 & 0.15 & 0.999 \\
\hline$x$ & PLSR & -12.47 & 0.35 & 0.996 \\
\hline$x$ & DQM & -9.11 & 0.99 & 0.980 \\
\hline Z & PLSR & -0.15 & 0.45 & 0.991 \\
\hline Z & DQM & -0.72 & 0.51 & 0.990 \\
\hline \multicolumn{5}{|l|}{$\mathrm{Z}$ as master } \\
\hline$Z$ & PLSR & 0.07 & 0.25 & 0.997 \\
\hline$z$ & $D Q M$ & -0.07 & 0.23 & 0.998 \\
\hline$x$ & PLSR & -8.93 & 0.44 & 0.995 \\
\hline$x$ & DQM & 4.25 & 1.08 & 0.977 \\
\hline$Y$ & PLSR & 0.11 & 0.26 & 0.999 \\
\hline$Y$ & DQM & 0.42 & 0.23 & 0.998 \\
\hline \multicolumn{5}{|l|}{$\mathrm{X}$ as master } \\
\hline$x$ & PLSR & 0.14 & 0.32 & 0.995 \\
\hline$x$ & $D Q M$ & 0.27 & 0.19 & 0.998 \\
\hline Z & PLSR & 14.32 & 0.63 & 0.990 \\
\hline Z & DQM & 6.14 & 0.45 & 0.992 \\
\hline$Y$ & PLSR & 15.77 & 0.64 & 1.000 \\
\hline$Y$ & DQM & -0.72 & 0.51 & 0.996 \\
\hline
\end{tabular}

\subsubsection{DQM and PLSR for Fruit Spectra}

Interactance spectra of 108 mango fruit of Kensington Pride cultivar were acquired on a F-750 instrument referred to as 'Master'. Two spectra were collected from two locations on each fruit. A 'test' population of 58 fruit was used for calibration ( $n=232, \mu=13.7$, $\sigma=1.6$ dry matter content (\%w/w DMC)), with two separate sets of 25 fruit of the same 
cultivar but different harvest dates used in validation (spectra $n=100, \mu=13.8, \sigma=1.5$

DMC). DQM and PLSR model statistics were compared.

After spectra collection, a $2 \mathrm{~cm}$ diameter by $1 \mathrm{~cm}$ deep core of flesh was taken at the point of spectra acquisition on each fruit, skin removed, and dry matter content (DMC) assessed gravimetrically following drying at $65^{\circ} \mathrm{C}$ to constant weight.

DQM models for fruit DMC based on first derivative spectra were slightly superior to those based on second derivative spectra (data shown in section 5.3.7). DQM models developed using the full region of $720-1020 \mathrm{~nm}$ with a gap limit of 14 and a smooth limit of 4 outperformed models based on the restricted range of $720 \mathrm{~nm}-981 \mathrm{~nm}$ with a gap limit of 7 and a smooth limit of 3 . The lower wavelength limit of $720 \mathrm{~nm}$ excluded the visible spectrum to avoid secondary relationships between plant pigments, fruit maturity and dry matter content. The restricted wavelength range for DQM was used to investigate what occurs when the routine is limited to a window slightly larger than the optimized PLS window. The relationship between dry matter content and pigment level varies with growing conditions and as such can vary year to year or farm to farm ${ }^{114}$. The upper limit of the wavelength range is constrained by instrumental noise, being at the edge of its spectral sensitivity. The improvement of the DQM model with use of an full wavelength range is ascribed to the effect of a large gap in reduction of noise in the higher wavelengths. 
PLSR models were slightly superior to the first derivative DQM model in the prediction of an independent test set (Table 5.4, also Table 5.12 and 5.13), with the notable exception of the precision of the result of the transfer of a master 2-term (720-1020 nm) DQM model to units $A$ and $B$ (Table 5.12).

Table 5.4, contrasts the performance of a 7 principle component PLSR calibration using the optimized wavelength range $732-936 \mathrm{~nm}$. PLSR slightly edged out the DQM calibration using the restricted wavelength range calibration. However, the full wavelength range DQM calibration (720-1020 $\mathrm{nm}$ search window) with the higher gap limit has a lower RMSEP and higher $\mathrm{R}_{\mathrm{val}}{ }^{2}$ than PLSR. The instrument selected for Table 5.4 was selected as the master instrument for exercise 4 presented in section 5.3.8.

Table 5.4. Performance of the master instrument for an optimized 7 factor PLSR model and DQM models based on two wavelength ranges in estimation of DMC of intact mango fruit (independent test set). DQM wavelengths are presented in the order of term 1 numerator, term 1 denominator, term 2 numerator, term 2 denominator, each with associated gap and smooth for the second derivative calculation.

\begin{tabular}{|c|c|c|c|}
\hline & $\mathrm{R}^{2}$ & Bias & RMSEP \\
\hline PLS (732-936 nm) & 0.898 & 0.009 & 0.503 \\
\hline $\begin{array}{l}\text { DQM (full range) : 2-term DQM } 981(10,1) \text {, } \\
894(5,1), 993(6,1), 996(12,4)\end{array}$ & 0.872 & 0.114 & 0.478 \\
\hline $\begin{array}{l}\text { DQM (restricted range) : 2-term DQM } 897 \\
(7,2), 900(3,2), 969(1,0), 900(1,0)\end{array}$ & 0.818 & 0.085 & 0.566 \\
\hline
\end{tabular}


5.3.7 Exercise 3 How the number of terms influence a single instrument DQM calibration For DMC of Mango

The optimal number of terms to be included in the DQM regression was investigated for the wavelength range $720-981 \mathrm{~nm}$ in context of performance of the model in prediction of an independent set (Table 5.5 and 5.6). Better prediction statistics were obtained with a model based on first rather than second derivative of absorbance spectra. The $\mathrm{R}_{\mathrm{val}}{ }^{2}$ suggests that a 2-term calibration is optimum, when the gap size is limited to 7 . The addition of a third term shows no significant increase in model performance when applied to the independent validation set.

Table 5.5. Influence of the number of terms for a 1st derivative calibration. DQM terms are reported as center wavelength (gap value, smooth value).

\begin{tabular}{lcc} 
& Numerator & Denominator \\
\hline Term \#1 & $897(7,2)$ & $900(3,2)$ \\
\hline Term \#2 & $869(1,0)$ & $900(1,0)$ \\
\hline Term \#3 & $819(7,2)$ & $852(4,2)$ \\
\cline { 2 - 3 } & $\mathbf{R}_{\text {val }}{ }^{\mathbf{2}}$ & RMSEP \\
\hline $\begin{array}{l}\text { 1-Term } \\
\text { Model }\end{array}$ & 0.775 & 0.616 \\
\hline $\begin{array}{l}\text { 2-Term } \\
\text { Model }\end{array}$ & 0.818 & 0.566 \\
\hline $\begin{array}{l}\text { 3-Term } \\
\text { Model }\end{array}$ & 0.814 & 0.567 \\
\hline
\end{tabular}


Table 5.6. Influence of the number of terms for a 2 nd derivative calibration. DQM terms are reported as center wavelength (gap value, smooth value).

\begin{tabular}{lcc} 
& Numerator & Denominator \\
\hline Term \#1 & $879(3,0)$ & $903(2,1)$ \\
\hline Term \#2 & $963(3,0)$ & $915(7,0)$ \\
\hline Term \#3 & $954(6,4)$ & $912(2,1)$ \\
\hline Term \#4 & $867(2,0)$ & $813(1,2)$ \\
\cline { 2 - 3 } $\mathbf{R}_{\text {val }}{ }^{2}$ & RMSEP \\
\hline $\begin{array}{l}\text { 1-Term } \\
\text { Model }\end{array}$ & 0.803 & 0.608 \\
\hline $\begin{array}{l}\text { 2-Term } \\
\text { Model }\end{array}$ & 0.791 & 0.637 \\
\hline $\begin{array}{l}\text { 3-Term } \\
\text { Model }\end{array}$ & 0.844 & 0.544 \\
\hline $\begin{array}{l}\text { 4-Term } \\
\text { Model }\end{array}$ & 0.86 & 0.528 \\
\hline
\end{tabular}

Increasing the gap size from 7 to 9 (Table 5.9) results in a 1-term model with an $R_{\text {val }}{ }^{2}$ of 0.84 . As shown by the decrease in $R^{2}$, subsequent terms for the 9 gap calibration are an over fit of the data. In contrast the 2-term model with an upper gap limit of 7 (Table 5.5) has an $\mathrm{R}_{\mathrm{val}}{ }^{2}$ of 0.59 . The first numerator of both calibrations take the weighted average of 9 wavelengths on either side of the center wavelength. Additionally the center wavelengths (894 $\mathrm{nm}$, gap=9, smoothing=0) and (897 nm, gap=7, smoothing=2) are adjacent pixels in the diode array. This implies that the wavelength range 864-924 nm, which contains $\mathrm{R}-\mathrm{CH}_{\mathrm{x}}$ and $\mathrm{R}-\mathrm{OH}$ overtones, are important for dry matter calibrations. 
Table 5.7. Influence of the number of terms for a 1st derivative calibration with gap limit 9. DQM terms are reported as center wavelength (gap value, smooth value).

\begin{tabular}{lcc} 
& Numerator & Denominator \\
\hline Term \#1 & $894(9,0)$ & $897(3,3)$ \\
\hline Term \#2 & $864(4,0)$ & $867(2,0)$ \\
\hline Term \#3 & $966(2,0)$ & $972(9,4)$ \\
\cline { 2 - 3 } & $\mathbf{R}_{\text {val }}{ }^{2}$ & RMSEP \\
\hline $\begin{array}{l}\text { 1-Term } \\
\text { Model }\end{array}$ & 0.83 & 0.53 \\
\hline $\begin{array}{l}\text { 2-Term } \\
\text { Model }\end{array}$ & 0.595 & 0.98 \\
\hline $\begin{array}{l}\text { 3-Term } \\
\text { Model }\end{array}$ & 0.63 & 0.92 \\
\hline
\end{tabular}

\subsubsection{Exercise 4 Mixing First and Second Derivative Terms}

Tables 5.8 and 5.9 investigate model performance when the order of the derivative in the numerator and denominator are varied. Table 5.8 shows the calibration statistics for a 2-term calibration with $1^{\text {st }}$ derivative numerators and $2^{\text {nd }}$ derivative denominators. Whereas, Table 5.9 shows the calibration statistics for a 2 -term calibration with a $2^{\text {nd }}$ derivative numerators and $1^{\text {st }}$ derivative denominators. Neither of the mixed order calibrations perform better than the single ordered calibrations in Table 5.6 and Table 5.7. 
Table 5.8. 2-term Second Derivative Numerator, First Derivative Denominator, gap limit 7. DQM terms are reported as derivative order center wavelength (gap value, smooth value).

\begin{tabular}{ccc} 
& Numerator & Denominator \\
\hline Term \#1 & 2D $888(3,1)$ & 1D 981 $(5,1)$ \\
\hline Term \#2 & 2D 837 $(6,1)$ & 1D 822 $(1,0)$ \\
\hline & Rval $^{2}$ & RMSEP \\
\cline { 2 - 3 } 2-Term Model & 0.673 & 1 \\
\cline { 2 - 3 } & &
\end{tabular}

Table 5.9. 2-term First Derivative Numerator, Second Derivative Denominator, gap limit 7. DQM terms are reported as derivative order center wavelength (gap value, smooth value).

\begin{tabular}{lcc} 
& Numerator & Denominator \\
\hline Term \#1 & 1D 891 (5,1) & 2D 888 (3,0) \\
\hline Term \#2 & 1D 801 (2,0) & 2D 867 (6,0) \\
\hline & Rval $^{2}$ & RMSEP \\
\cline { 2 - 3 } 2-Term Model & 0.779 & 0.66 \\
\cline { 2 - 3 } & &
\end{tabular}

\subsubsection{Exercise 5 Development of a Global 6 instrument DQM Calibration}

A global calibration consisting of 1104 spectra, was collected on 6 instruments. A second independent dataset from instrument master was used as validation for the DQM routine. Based on the results of 5.3.7 only $1^{\text {st }}$ derivative terms were considered for global calibration. 
As shown by the low $\mathrm{R}^{2}$, in Table 5.10 , the variability in the spectra of the global dataset is insufficiently modeled by a 2-term DQM regression. Increasing the calibration to a 4term allows for DQM to better span the variation in the multi-instrument dataset and result in calibration statistics similar to those found previously for a single instrument calibration. Table 5.11 shows how the number of terms improves the full range (750$1020 \mathrm{~nm}$ ) wavelength calibration.

Table 5.10. Influence of the number of terms for $720-981 \mathrm{~nm}$ Global $1^{\text {st }}$ derivative calibration for DMC. Model developed using restricted wavelength range with first derivative of absorbance spectra for instrument $F$ (master instrument). DQM terms are reported as center wavelength (gap value, smooth value).

\begin{tabular}{lcc} 
& Numerator & Denominator \\
\hline Term \#1 & $972(2,0)$ & $933(1,0)$ \\
\hline Term \#2 & $975(6,0)$ & $906(6,0)$ \\
\hline Term \#3 & $810(2,0)$ & $881(4,3)$ \\
\hline Term \#4 & $894(4,3)$ & $894(7,3)$ \\
\hline & $\mathbf{R}_{\text {val }}{ }^{2}$ & RMSEP \\
\cline { 2 - 3 } 1-Term Model & 0.3 & 1.12 \\
\cline { 2 - 3 } 2-Term Model & 0.45 & 0.98 \\
\cline { 2 - 3 } 3-Term Model & 0.623 & 0.81 \\
\cline { 2 - 3 } 4-Term Model & 0.8 & 0.69
\end{tabular}


Table 5.11. Influence of the number of terms for an 750-1020 $\mathrm{nm}$ Global $1^{\text {st }}$ derivative calibration for DMC. Based on 1st derivative spectra for instrument F Master. DQM terms are reported as center wavelength (gap value, smooth value).

\begin{tabular}{lcc} 
& Numerator & Denominator \\
\hline Term \#1 & $972(2,0)$ & $933(1,0)$ \\
\hline Term \#2 & $978(8,0)$ & $903(1,0)$ \\
\hline Term \#3 & $813(1,0)$ & $981(1,0)$ \\
\hline Term \#4 & $891(3,1)$ & $885(13,0)$ \\
\hline & $\mathbf{R}_{\text {val }^{2}}$ & RMSEP \\
\cline { 2 - 3 } 1-Term Model & 0.3 & 1.12 \\
\cline { 2 - 3 } 2-Term Model & 0.509 & 0.92 \\
\cline { 2 - 3 } 3-Term Model & 0.631 & 0.798 \\
4-Term Model & 0.8 & 0.43
\end{tabular}

\subsubsection{Exercise 6 - Calibration transfer of PLSR and DQM models for mangos}

For all calibration transfer exercises, a population of 25 Kensington Pride cultivar mango fruit ( $n=100, \mu=14.4, \sigma=1.3 \mathrm{DMC}$ ) was used, but having a different harvest date to the calibration and validation sets; and for further PDS transfer functions, spectra were collected of: (i) a set of cultivar Calypso fruit ( $n=280, \mu=16.6, \sigma=1.0$ DMC); (ii) R2E2 fruit ( $n=300, \mu=14.7, \sigma=1.4$ DMC) and (iii) apple fruit ( $n=176, \mu=12.9, \sigma=1.3$ DMC). These were acquired on all six instruments as per the method described above.

The PLSR models developed for the master unit were used 'direct' in prediction using unaltered spectra of other slave units. Results compared to those for several instrument standardization techniques for the PLSR model, involving either spectral correction (PDS, DSA), global models or model updating (Table 5.12 and Table 5.13). For the DSA technique, the mean difference spectra were calculated using spectra of the Kensington 
Pride fruit transfer set, and used in adjustment of all validation set spectra. This was also combined with the new pixel-to-wavelength assignments, as described above. PDS was performed using the four different transfer sets listed above.

Several PLSR MU and global model approaches for were trialed using the Kensington Pride transfer set: (i) a global model with all spectra of the calibration sets of units $A$ to D (with X included only on its own); (ii) same as (i) with increased PLS factors (iii) a master unit based model, updated with spectra from the transfer sets of units $A$ to $D$, (iv) same as (iii) with increased PLS factors, (v) model based on spectra of the master unit updated with spectra from the transfer set of the respective slave unit, (vi) a model based on spectra of the master unit, updated with spectra from the transfer set of the slave unit only, but predicted DMC values from the master unit, (vii) slave unit based model, using spectra from the slave transfer set and predicted DMC values from the master unit. The last two methods seek to improve practicality of implementation by avoiding the need for reference method analyses.

To compare the transfer of PLSR with DQM, the DQM model developed on the master was used directly on the slave instruments, as well as global models as per the PLSR models above. The calibration transfer dataset from the master instrument was used as validation for the DQM routine.

The performance of models created on one unit and used in prediction of spectra collected on another unit was impacted both in terms of $\mathrm{R}^{2}$ (and corresponding SEP - 
data not shown) and bias (Table 5.12 'Direct' results). The PLSR DSA method improved bias for all slave unit predictions, although bias magnitude $>0.5$ occurred in two cases, without affecting $\mathrm{R}^{2}$. In these two cases, the difference spectra, which was calculated using the transfer set, did not match the difference of the validation sets. The new pixel to wavelength assignment combined with DSA improved $R^{2}$ of unit $X$, as expected due to the discrepancy in wavelength calibration of this unit. All other transfer methods trialed were successful in decreasing bias (Table 5.13).

Prediction $\mathrm{R}^{2}$ was most consistently improved using PDS (based on a transfer set of the same variety of fruit as the calibration model), the Global (10 factors) and the Master model global updating (10 factors) approaches. However, the use of the same fruit variety for the PDS transfer set as used for the calibration set can be impractical due to seasonal availability of fruit. PDS using a related variety (Calypso) was successful across all units, however use of fruit of the R2E2 variety for the transfer set proved unsuccessful on some units. Surprising, the use of apple spectra as the PDS training set proved quite successful, except on unit X. This latter result is believed to be due to the drastic difference in wavelength alignment of the units.

Except for unit $\mathrm{X}$, a global model using spectra from all instruments gave satisfactory performance when the number of PLS factors was increased to 10, allowing the PLS calibration to model for instrument difference. 
The performance of models based on individual unit model updating (model based on master calibration set plus transfer set from slave unit) was also comparable to that of PDS based models. Interestingly, use of DMC values estimated by the master instrument (with no wet chemistry performed) for the transfer set (spectra collected on the slave unit), gave acceptable performance. This approach has the advantage of being able to be implemented rapidly in field situations.

For the DQM calibration, the full range $(720-1020 \mathrm{~nm})$ with the higher gap limit had marginally better direct transfer results than the restricted range $(750-981 \mathrm{~nm})$. It is strange that instrument A was well predicted by the initial model and poorly predicted by the full wavelength range model. 
Table 5.12. DQM vs PLS calibration transfer results. $R^{2}$ for fruit DMC models developed on a given unit using spectra of a calibration set of Kensington Pride fruit from respective units, used in prediction of an independent population of fruit for the slave units $A, B, C, D$ and $X$; using $(A)$ single instrument PLSR calibrations; (B) for a second derivative PLSR model transferred using various methods from the F-750 unit master (unit F); (C) for a first derivative based DQM model directly transferred from the master (unit F) to the slave units, and (D) a Global DQM model. For a given unit, highest results $R^{2}$ values within 0.2 units are shown in bold.

\begin{tabular}{|c|c|c|c|c|c|c|}
\hline \multirow[b]{3}{*}{ A. PLSR model on single unit } & \multicolumn{5}{|c|}{$\mathbf{R}^{2}$} & \multirow[b]{2}{*}{$\mathrm{X}$} \\
\hline & $\begin{array}{l}\text { Master } \\
\text { (Y) }\end{array}$ & $A$ & B & C & $\mathrm{D}$ & \\
\hline & 0.90 & 0.88 & 0.84 & 0.89 & 0.90 & 0.91 \\
\hline \multicolumn{7}{|l|}{$\begin{array}{l}\text { B. PLSR model transfer between } \\
\text { units }\end{array}$} \\
\hline Direct & 0.90 & 0.78 & 0.53 & 0.90 & 0.36 & 0.57 \\
\hline DSA & N/A & 0.78 & 0.53 & 0.90 & 0.36 & 0.57 \\
\hline DSA + wavelength reassignment & N/A & 0.82 & 0.51 & 0.86 & 0.56 & 0.82 \\
\hline PDS & N/A & 0.87 & 0.84 & 0.89 & 0.88 & 0.91 \\
\hline PDS (Calypso) & $\mathrm{N} / \mathrm{A}$ & N/A & 0.82 & 0.88 & 0.85 & 0.89 \\
\hline PDS (R2E2) & N/A & N/A & 0.41 & 0.82 & 0.86 & 0.92 \\
\hline PDS (Apple) & N/A & 0.89 & 0.83 & 0.88 & 0.87 & 0.65 \\
\hline Global (7 f) & 0.83 & 0.84 & 0.75 & 0.85 & 0.79 & 0.75 \\
\hline Global (10 f) & 0.90 & 0.89 & 0.85 & 0.92 & 0.86 & 0.79 \\
\hline Master model, global updating (7 f) & 0.86 & 0.86 & 0.82 & 0.88 & 0.85 & 0.77 \\
\hline Master model, global updating (10 f) & 0.92 & 0.88 & 0.86 & 0.92 & 0.87 & 0.81 \\
\hline Individual model updating & 0.91 & 0.86 & 0.83 & 0.89 & 0.84 & 0.87 \\
\hline $\begin{array}{l}\text { Individual model updating - master } \\
\text { predicted DMC }\end{array}$ & 0.90 & 0.85 & 0.82 & 0.87 & 0.83 & 0.86 \\
\hline $\begin{array}{l}\text { Individual models updated using } \\
\text { master predicted DMC }\end{array}$ & 0.89 & 0.85 & 0.82 & 0.84 & 0.84 & 0.81 \\
\hline \multicolumn{7}{|l|}{ C. DQM model transfer } \\
\hline 2-term restricted range & 0.86 & 0.84 & 0.76 & 0.81 & 0.80 & 0.72 \\
\hline 2-term full range & 0.88 & 0.97 & 0.98 & 0.85 & 0.85 & 0.81 \\
\hline \multicolumn{7}{|l|}{ D. DQM Global model transfer } \\
\hline 4-term restricted range & 0.87 & 0.76 & 0.75 & 0.79 & 0.78 & 0.80 \\
\hline 4-term full range & 0.85 & 0.78 & 0.76 & 0.83 & 0.80 & 0.83 \\
\hline
\end{tabular}


Table 5.13. DQM vs PLS calibration transfer results. Same as Table 5.12, with bias instead of $\mathrm{R}^{2}$ reported. For a given unit, lowest bias values within $0.2 \% \mathrm{w} / \mathrm{w}$ are shown in bold.

\begin{tabular}{|c|c|c|c|c|c|c|}
\hline \multirow[b]{3}{*}{ A. Calibration on single unit } & \multicolumn{3}{|r|}{ Bias } & \multirow{2}{*}{$\frac{1 \%}{\mathrm{C}}$} & \multicolumn{2}{|l|}{$w / w)$} \\
\hline & $\begin{array}{c}\text { Master } \\
(\mathrm{Y})\end{array}$ & A & B & & $\mathrm{D}$ & $x$ \\
\hline & 0.01 & -0.14 & 0.16 & 0.12 & 0.05 & -0.02 \\
\hline \multicolumn{7}{|l|}{ B. Transfer between units } \\
\hline Direct & 0.01 & 1.94 & -0.26 & -2.81 & -1.68 & -17.6 \\
\hline DSA & $\mathrm{N} / \mathrm{A}$ & -0.37 & -0.57 & 0.22 & -0.63 & 0.03 \\
\hline DSA + wavelength reassignment & N/A & 0.18 & -0.27 & 0.38 & -0.17 & 0.01 \\
\hline PDS & N/A & 0.01 & 0.09 & 0.14 & 0.20 & -0.01 \\
\hline PDS (Calypso) & N/A & $\mathrm{N} / \mathrm{A}$ & 0.04 & -0.07 & -0.09 & -0.66 \\
\hline PDS (R2E2) & N/A & N/A & 0.40 & -0.87 & -0.03 & -0.30 \\
\hline PDS (Apple) & N/A & -0.20 & 0.11 & 0.12 & 0.14 & -1.20 \\
\hline Global (7 f) & -0.03 & 0.30 & 0.42 & -0.08 & -0.13 & 0.38 \\
\hline Global (10 PCs) & 0.02 & -0.04 & 0.19 & 0.07 & -0.16 & 0.37 \\
\hline Master model, global updating (7 f) & 0.14 & 0.14 & 0.36 & -0.08 & -0.07 & 0.43 \\
\hline $\begin{array}{l}\text { Master model, global updating (10 } \\
\text { PCs) }\end{array}$ & -0.03 & -0.15 & 0.08 & 0.07 & -0.30 & 0.23 \\
\hline Individual model updating & -0.09 & -0.42 & -0.22 & -0.05 & -0.21 & 0.02 \\
\hline $\begin{array}{l}\text { Individual model updated master } \\
\text { predicted DMC }\end{array}$ & 0.01 & -0.22 & -0.07 & 0.10 & -0.07 & 0.12 \\
\hline $\begin{array}{l}\text { Individual models updated using } \\
\text { master predicted DMC }\end{array}$ & -0.01 & 0.14 & 0.14 & 0.07 & -0.03 & -0.24 \\
\hline \multicolumn{7}{|l|}{ C. DQM model transfer } \\
\hline 2-term restricted range & 0.07 & 2.63 & 0.53 & 0.13 & -2.35 & -5.91 \\
\hline 2-term full range & 0.00 & 0.55 & 0.66 & -1.79 & -2.08 & -11.5 \\
\hline \multicolumn{7}{|l|}{ D. DQM Global model transfer } \\
\hline 4-term restricted range & 0.52 & 0.32 & 0.46 & 0.29 & -0.20 & -5.73 \\
\hline 4-term full range & 0.56 & 0.42 & 0.44 & -0.02 & -0.37 & -3.95 \\
\hline
\end{tabular}

\subsection{Conclusion}

The DQM1. routine produced a calibration that was as statistically equivalent to PLSR under cross-validation for a binary mixture of sugar water. The DQM program is based on the work of Karl Norris on Gap and Smoothing derivatives. The DQM routine introduces the idea of a Gap-Smooth derivative as an alternative approach to iteratively find to 
optimize the Gap-Segments derivatives used in a Multilinear Regressions. For single instrument calibrations of Kingston pride mangoes, DQM has been found to produce calibrations with lower SEC than PLSR.

The best calibration transfer results ( $\mathrm{R}^{2}$ and bias) were obtained using PLSR+PDS using the same variety of fruit in calibration and transfer sets. This method is impractical due to seasonal availability of fruit. If the wavelength accuracy of the slave unit(s) is satisfactory, apples could be used to transfer the mango calibrations all year round (i.e. 'out of mango season'). A global model across units or model updating, using reference values estimated using the master unit can be utilized in field situations. Both the DQM global model and the single instrument model PLSR. The global DQM model was not able to overcome the wavelength misalignment of unit $\mathrm{X}$. 


\section{Chapter 6}

Conclusion and Future Work 
Both industrial and academic postharvest researchers need reliable sensors to monitor the maturation and senescence processes of fresh produce ${ }^{2,115}$. Many of their needs can be met by two different but complementary methods; spectroscopy to monitor the internal changes in fruit and electrochemical gas sensing for ethylene emitted by fruit. The objective of the conducted research was to address limitations in both of these sensing technologies with specific focus towards the needs of postharvest researchers. By advancing the state of the art of these sensing technologies, postharvest researchers can now collect more accurate data on how fruit and other fresh produce change as they pass through the supply chain.

The performance of an electrochemical sensor was optimized for the detection of ethylene emitted by apples through the development of an inline aqueous filter that trapped polar organic compounds. The removal of polar organic compounds from the airstream prior to the sensor resulted in a $50 \%$ improvement in the accuracy of electrochemical readings as confirmed by GC-FID. To avoid saturation of the trap, this study recommended that the inline filter be periodically regenerated by means of a closed loop flow of potassium permanganate. The frequency and duration of the regeneration period is application specific. Additionally, an economical alternative to cylinders of calibration gas was presented.

The performance of handheld commercial spectrometers for the fruit industry was evaluated under dynamic conditions. Changes to spectral collect protocols were 
suggested to reduce the influence of fluctuating environmental temperature and background or self-illumination of the sample. Additionally the issue of transferring calibrations between portable diode array spectrometers was evaluated. Best results $\left(\mathrm{R}^{2}\right.$ and bias) for calibration transfer were obtained using Piecewise Direct Standardization using the same variety of fruit in calibration and transfer sets. This method is impractical due to seasonal availability of fruit. The practical solution for model transfer is Model Updating at the beginning of each season using the predicted values of the master unit instead of new wet chemistry reference values. The Derivative Quotient Method of spectral regression is no better than Model Updating for calibration transfer between shortwave handheld diode array spectrometers.

With the increasing availability of the internet of things, the demand for portable sensors in the postharvest industry will continue to grow. Future work should focus on meeting industry's need of low cost sensors with sufficient accuracy to measure the various stages of fruit maturation and storage. The role of LED and MEMS based spectrometer solutions will continue to be a research interest for those developing new postharvest technologies. In addition, consideration should be given to the role of information systems and geotagging data collected by these new sensors. 
References

1. Apple Industry Statistics http://usapple.org/all-about-apples/apple-industrystatistics/ (2018).

2. Ivanov S, Bhargava K and Donnelly W. Precision Farming: Sensor Analytics. IEEE Intelligent Systems 2015; 30: 76-80. DOI: 10.1109/mis.2015.67.

3. Herold B, Truppel I, Zude M, et al. Spectral Measurements on 'Elstar' Apples during Fruit Development on the Tree. Biosystems Engineering 2005; 91: 173-182. DOI: 10.1016/j.biosystemseng.2005.03.005.

4. Alexander $L$ and Grierson $D$. Ethylene biosynthesis and action in tomato: a model for climacteric fruit ripening. J Exp Bot 2002; 53: 2039-2055. 2002/09/27. DOI: 10.1093/jxb/erf072.

5. Allroza Shekarriz WLA, Daniel James Faulkner, Christopher M. Ward, Debra M. Gilbuena Tree fruit postharvest chemical sensor. Patent 7,560,013, United States, 2007.

6. Mitcham EJ, Crisosto $\mathrm{CH}$ and Kader AA. Recommendations for Maintaining Postharvest Quality - Gala Apple, http://postharvest.ucdavis.edu/Commodity Resources/Fact Sheets/Datastores/Fruit E nglish/?uid=2\&ds=798 (1997, accessed December 2017).

7. Holmes La. Storage technology for apples and pears.: Dept of natural resources and environment, Victoria, Australia, 2000.

8. Saltveit ME. Effect of ethylene on quality of fresh fruits and vegetables. Postharvest Biology and Technology 1999; 15: 279-292. DOI: 10.1016/s09255214(98)00091-x.

9. Johnston JW, Gunaseelan K, Pidakala P, et al. Co-ordination of early and late ripening events in apples is regulated through differential sensitivities to ethylene. $J$ Exp Bot 2009; 60: 2689-2699. 2009/05/12. DOI: 10.1093/jxb/erp122.

10. Harker FR, Kupferman EM, Marin AB, et al. Eating quality standards for apples based on consumer preferences. Postharvest Biology and Technology 2008; 50: 70-78. DOI: 10.1016/j.postharvbio.2008.03.020.

11. Wills RB and Golding JB. Reduction of energy usage in postharvest horticulture through management of ethylene. J Sci Food Agric 2015; 95: 1379-1384. 2014/09/27. DOI: 10.1002/jsfa.6930.

12. Cristescu SM, Mandon J, Arslanov D, et al. Current methods for detecting ethylene in plants. Ann Bot 2013; 111: 347-360. 2012/12/18. DOI: 10.1093/aob/mcs259. 13. Caprioli $F$ and Quercia L. Ethylene detection methods in post-harvest technology: A review. Sensors and Actuators B: Chemical 2014; 203: 187-196. DOI: 10.1016/j.snb.2014.06.109.

14. Rowan DD, Hunt MB, Dimouro A, et al. Profiling fruit volatiles in the progeny of a 'Royal Gala' x 'Granny Smith' apple (Malus x domestica) cross. J Agric Food Chem 2009; 57: 7953-7961. 2009/08/21. DOI: 10.1021/jf901678v. 
15. Zevenbergen MA, Wouters D, Dam VA, et al. Electrochemical sensing of ethylene employing a thin ionic-liquid layer. Anal Chem 2011; 83: 6300-6307. 2011/07/05. DOI: 10.1021/ac2009756.

16. Jordan LR, Hauser PC and Dawson GA. Amperometric Sensor for Monitoring Ethylene. Analytical Chemistry 1997; 69: 558-562. DOI: 10.1021/ac9610117.

17. Hodgson AW, Jacquinot P and Hauser PC. Amperometric sensing of ethylene oxide in the gas phase. Anal Chem 2000; 72: 2206-2210. 2000/06/14. DOI: 10.1021/ac991247l.

18. Hodgson AWE, Jacquinot P, Jordan LR, et al. Amperometric Gas Sensors of High Sensitivity. Electroanalysis 1999; 11: 782-787. DOI: 10.1002/(sici)15214109(199907)11:10/11<782::Aid-elan782>3.0.Co;2-s.

19. SPECIFICATION SHEET FOR ETHYLENE OXIDE SENSOR TYPE ETO/M-10, http://www.membrapor.ch/sheet/ETO-M-10.pdf (2010).

20. Hossain M, Saffell J and Baron R. Differentiating NO2 and $\mathrm{O} 3$ at Low Cost Air Quality Amperometric Gas Sensors. ACS Sensors 2016; 1: 1291-1294. DOI: 10.1021/acssensors.6b00603.

21. Komthong P, Hayakawa S, Katoh T, et al. Determination of potent odorants in apple by headspace gas dilution analysis. LWT - Food Science and Technology 2006; 39: 472-478. DOI: 10.1016/j.Iwt.2005.03.003.

22. Beaudry CDGaRM. Determination of the low oxygen limit for several commercial apple cultivars by respiratory quotient breakpoint. Postharvest Biology and Technology 1993; 3: 259-267.

23. McGlone VA, Jordan RB, Seelye R, et al. Dry-matter-a better predictor of the post-storage soluble solids in apples? Postharvest Biology and Technology 2003; 28: 431-435. DOI: 10.1016/s0925-5214(02)00207-7.

24. McGlone VA and Kawano S. Firmness, dry-matter and soluble-solids assessment of postharvest kiwifruit by NIR spectroscopy. Postharvest Biology and Technology 1998; 13: 131-141. DOI: 10.1016/s0925-5214(98)00007-6.

25. Palmer JW, Harker FR, Tustin DS, et al. Fruit dry matter concentration: a new quality metric for apples. J Sci Food Agric 2010; 90: 2586-2594. 2010/08/19. DOI: 10.1002/jsfa.4125.

26. Zude M. Optimum Harvest Date Determination for Apples Using Spectral Analysis. Die Gartenbauwissenschaft 2002.

27. Bessemans N, Verboven P, Verlinden BE, et al. A novel type of dynamic controlled atmosphere storage based on the respiratory quotient (RQ-DCA). Postharvest Biology and Technology 2016; 115: 91-102. DOI: 10.1016/j.postharvbio.2015.12.019. 28. Theologis A. One rotten apple spoils the whole bushel: the role of ethylene in fruit ripening. Cell 1992; 70: 181-184. 1992/07/24. DOI: 10.1016/0092-8674(92)90093-R. 29. Blanke MM. Reducing ethylene levels along the food supply chain: a key to reducing food waste? J Sci Food Agric 2014; 94: 2357-2361. 2014/03/22. DOI: 10.1002/jsfa.6660. 
30. Park DS and Jeong CS. Fabrication and optimization of ethylene-producing tablet. Korean Journal of Food Preservation 2016; 23: 162-165. DOI: 10.11002/kjfp.2016.23.2.162.

31. Lerud RM, Beseau D, Hale CM, et al. Optimizing the performance of a commercial electrochemical ethylene sensor via controlled ethylene generation in situ. Sensors and Actuators B: Chemical 2019; 281: 535-541. DOI: 10.1016/j.snb.2018.09.127. 32. Hayes CJ, Greensill CV and Walsh KB. Temporal and Environmental Sensitivity of a Photodiode Array Spectrophometric System. Journal of Near Infrared Spectroscopy 2014; 22: 297-304. DOI: 10.1255/jnirs.1112.

33. Hayes $\mathrm{C}$. The influence of visible-shortwave NIR spectroscopy hardware components on prediction of fruit attributes. Central Queensland University, (Unpublished), 2016.

34. Wold H. Non-linear estimation by iterative least squares procedures. Research Papers in Statistics 1966: 411-444.

35. Norris KH. Interactions among Instrument Bandpass, Instrument Noise, SampleAbsorber Bandwidth and Calibration Error. NIR news 2017; 9: 3-5. DOI: 10.1255/nirn.474.

36. Norris K, and P. Williams. Optimization of Mathematical Treatments of Raw Near-Infrared Signal in the Measurement of Protein in Hard Red Spring Wheat. I. Influence of Particle Size. Cereal Chem 1984; 61: 158-165.

37. Lerud R. The Importance of the Reference Method Accuracy and Why it is Important Not to Cut Corners While Developing a Model. NIR news 2016; 27: 21-22. DOI: 10.1255/nirn.1603.

38. Lerud R. An Experiment on the Effects of Detector Design and Thermal Properties as a Function of Noise. NIR news 2016; 27: 25-27. DOI: 10.1255/nirn.1652. 39. Lerud R. Demystifying the Black Box: Improving Detection Limits. NIR news 2017; 26: 14-15. DOI: 10.1255/nirn.1523.

40. Klein I, Lavee S and Ben-Tal Y. Effect of water vapor pressure on the thermal decomposition of 2-chloroethylphosphonic Acid. Plant Physiol 1979; 63: 474-477. 1979/03/01. DOI: http://dx.doi.org/10.1104/pp.63.3.474.

41. Warner HL and Leopold AC. Ethylene evolution from 2-chloroethylphosphonic Acid. Plant Physiol 1969; 44: 156-158. 1969/01/01. DOI: 10.1104/pp.44.1.156.

42. Klein I, Lavee $S$ and Ben-Tal Y. Effect of Water Vapor Pressure on the Thermal Decomposition of 2-Chloroethylphosphonic Acid. Plant Physiology 1979; 63: 474-477. DOI: $10.1104 / p p .63 .3 .474$.

43. Yang SF. Ethylene evolution from 2-chloroethylphosphonic Acid. Plant Physiol 1969; 44: 1203-1204. 1969/08/01. DOI: 10.1104/pp.44.8.1203.

44. Zhang W and Wen CK. Preparation of ethylene gas and comparison of ethylene responses induced by ethylene, ACC, and ethephon. Plant Physiol Biochem 2010; 48: 4553. 2009/10/20. DOI: 10.1016/j.plaphy.2009.10.002. 
45. Maynard JA and Swan JM. Organophosphorus Compounds. I. 2-

Chloroalkylphosphonic Acids as Phosphorylating Agents. Australian Journal of Chemistry 1963; 16: 596-608. DOI: 10.1071/ch9630596.

46. ToolBox E. Solubility of Gases in Water, https://www.engineeringtoolbox.com/gases-solubility-water-d 1148.htm (2008, accessed August 18th, 2018).

47. Espino-Diaz M, Sepulveda DR, Gonzalez-Aguilar G, et al. Biochemistry of Apple Aroma: A Review. Food Technol Biotechnol 2016; 54: 375-397. 2017/01/25. DOI: 10.17113/ftb.54.04.16.4248.

48. Yahia EM. Fruit and Vegetable Phytochemicals: Chemistry and Human Health. John Wiley \& Sons, Inc, 2017.

49. Warburton and Richard P. Filter for gas sensor. Patent 7,491,547, United States, 2009.

50. Jordan LR, Hauser PC and Dawson GA. Portable Trap-Sensor System for Monitoring Low Levels of Ethylene. The Analyst 1997: 811-814. DOI: 10.1039/A702444D.

51. Ethylene Sensor Zero Calibration, https://felixinstruments.com/static/media/uploads/manuals/f900 ethylenezerocalibrati on.pdf (2015).

52. Takahara I, Saito M, Inaba M, et al. Dehydration of Ethanol into Ethylene over Solid Acid Catalysts. Catalysis Letters 2005; 105: 249-252. DOI: 10.1007/s10562-0058698-1.

53. Nogueira JM, Fernandes PJ and Nascimento AM. Composition of volatiles of banana cultivars from Madeira Island. Phytochem Anal 2003; 14: 87-90. 2003/04/16. DOI: 10.1002/pca.691.

54. Watkins CB, Bowen JH and Walker VJ. Assessment of ethylene production by apple cultivars in relation to commercial harvest dates. New Zealand Journal of Crop and Horticultural Science 1989; 17: 327-331. DOI: 10.1080/01140671.1989.10428052.

55. J. Guthrie KW. Non-invasive assessment of pinapple and mango fruit quality using near infra-red spectroscopy. Australian Journal of Experimental Agriculture 1997; 37: 253-263.

56. DAHM DJ, \& DAHM, K. D. Interpreting diffuse reflectance and transmittance: $a$ theoretical introduction to absorption spectroscopy of scattering materials. Chichester: IM Publications LLP, 2007.

57. Rinnan $\AA$, Berg Fvd and Engelsen SB. Review of the most common pre-processing techniques for near-infrared spectra. TrAC Trends in Analytical Chemistry 2009; 28: 1201-1222. DOI: 10.1016/j.trac.2009.07.007.

58. Barnes RJ, Dhanoa MS and Lister SJ. Standard Normal Variate Transformation and De-trending of Near-Infrared Diffuse Reflectance Spectra. Applied Spectroscopy 1989; 43: 772-777. 
59. Martens $\mathrm{H}$, Nielsen JP and Engelsen SB. Light scattering and light absorbance separated by extended multiplicative signal correction. application to near-infrared transmission analysis of powder mixtures. Anal Chem 2003; 75: 394-404. 2003/02/15. DOI: 10.1021/ac020194w.

60. Fearn T. Multiplicative Pre-Treatments Spoil Beer-Lambert. NIR news 2016; 27: 25-26. DOI: 10.1255/nirn.1596.

61. Fearn T, Riccioli C, Garrido-Varo A, et al. On the geometry of SNV and MSC. Chemometrics and Intelligent Laboratory Systems 2009; 96: 22-26. DOI: 10.1016/j.chemolab.2008.11.006.

62. Difoggio R. Examination of Some Misconceptions about Near-Infrared Analysis. Applied Spectroscopy 2016; 49: 67-75. DOI: 10.1366/0003702953963247.

63. Cen $\mathrm{H}$ and $\mathrm{He} \mathrm{Y}$. Theory and application of near infrared reflectance spectroscopy in determination of food quality. Trends in Food Science \& Technology 2007; 18: 72-83. DOI: 10.1016/j.tifs.2006.09.003.

64. Rodriguez-Otero JL, Hermida M and Cepeda A. Determination of fat, protein, and total solids in cheese by near-infrared reflectance spectroscopy. J AOAC Int 1995; 78: 802-806. 1995/05/01.

65. Nicolaï BM, Beullens K, Bobelyn E, et al. Nondestructive measurement of fruit and vegetable quality by means of NIR spectroscopy: A review. Postharvest Biology and Technology 2007; 46: 99-118. DOI: 10.1016/j.postharvbio.2007.06.024.

66. Wold S, Sjöström M and Eriksson L. PLS-regression: a basic tool of chemometrics. Chemometrics and Intelligent Laboratory Systems 2001; 58: 109-130. DOI: 10.1016/s0169-7439(01)00155-1.

67. Xu Q-S, Liang Y-Z and Shen H-L. Generalized PLS regression. Journal of Chemometrics 2001; 15: 135-148. DOI: 10.1002/cem.605.

68. Phatak $A$ and De Jong $S$. The geometry of partial least squares. Journal of Chemometrics 1997; 11: 311-338. DOI: 10.1002/(sici)1099-128x(199707)11:4<311::Aidcem478>3.0.Co;2-4.

69. Geladi P and Kowalski BR. Partial least-squares regression: a tutorial. Analytica Chimica Acta 1986; 185: 1-17. DOI: 10.1016/0003-2670(86)80028-9.

70. Wold S, Sjöström M, Carlson R, et al. Multivariate design. Analytica Chimica Acta 1986; 191: 17-32. DOI: 10.1016/s0003-2670(00)86294-7.

71. Mortensen PP. Myth: A Partial Least Squares Calibration Model Can Never Be More Precise Than the Reference Method.... NIR news 2014; 25: 20-22. DOI: 10.1255/nirn.1438.

72. Schaare PN and Fraser DG. Comparison of reflectance, interactance and transmission modes of visible-near infrared spectroscopy for measuring internal properties of kiwifruit (Actinidia chinensis). Postharvest Biology and Technology 2000; 20: 175-184. DOI: 10.1016/s0925-5214(00)00130-7.

73. T. Temma KHaFS. Development of a portable near infrared sugar-measuring instrument. J Near Infrared Spectroscopy 2002; 10: 77-83. 
74. al. JMe. LED-based NIR spectrometer module for hand-held and process analyser applications. Sensors and Actuators B: Chemical 1998.

75. Yuan L-m, Cai J-r, Sun L, et al. Nondestructive Measurement of Soluble Solids Content in Apples by a Portable Fruit Analyzer. Food Analytical Methods 2015; 9: 785794. DOI: 10.1007/s12161-015-0251-2.

76. Hale GM and Querry MR. Optical Constants of Water in the 200-nm to 200microm Wavelength Region. Appl Opt 1973; 12: 555-563. 1973/03/01. DOI: 10.1364/AO.12.000555.

77. User:Kebes. Absorption spectrum of liquid water. Wikipedia, 2008.

78. Metrohm AG: Herisay S. Metrohm Monograph. 2016.

79. Clancy PJ. The Effects of Stray Light and Saturation on NIR Spectra. NIR news 2001; 12: 7-9.

80. Wulfert F, Kok WT and Smilde AK. Influence of temperature on vibrational spectra and consequences for the predictive ability of multivariate models. Anal Chem 1998; 70: 1761-1767. 1998/05/01. DOI: 10.1021/ac9709920.

81. Chen ZP, Morris J and Martin E. Correction of temperature-induced spectral variations by loading space standardization. Anal Chem 2005; 77: 1376-1384. 2005/03/01. DOI: 10.1021/ac040119g.

82. Wulfert F, Kok WT, de Noord OE, et al. Correction of temperature-induced spectral variation by continuous piecewise direct standardization. Anal Chem 2000; 72: 1639-1644. 2000/04/14. DOI: 10.1021/ac9906835.

83. Anderson NT, Subedi PP and Walsh KB. Manipulation of mango fruit dry matter content to improve eating quality. Scientia Horticulturae 2017; 226: 316-321. DOI: 10.1016/j.scienta.2017.09.001.

84. Greensill C, \& Walsh, K. Optimization of Instrumentation Precision and Wavelength Resolution for the Performance of NIR Calibrations of Sucrose in a WaterCellulose Matrix. Applied Spectroscopy 2000; 54: 426-430.

85. Felix I. F-750 Mango Model Building Standard Operating Procedure. 2015.

86. Subedi PP, Walsh KB and Owens G. Prediction of mango eating quality at harvest using short-wave near infrared spectrometry. Postharvest Biology and Technology 2007; 43: 326-334. DOI: 10.1016/j.postharvbio.2006.09.012.

87. Walsh KB. The ratio of specular to diffuse radiation detected using a "shadow probe" configuration. In: NIR 20132013.

88. Savitzky AG, Marcel JE. Smoothing and differentiation of data by simplified least squares procedures. Analytical chemistry 1964; 36: 1627-1639.

89. Davidson MW. Tungsten Lamp Profile as Function of Blackbody. In: tungstenlampsfigure1.jpg, (ed.). National High Magnetic Field Laboratory - The Florida State University, 2017.

90. Characteristic and use of NMOS Linear Image Sensors. Hamamatsu, 2017. 
91. Kaur H, Künnemeyer $\mathrm{R}$ and McGlone A. Comparison of hand-held near infrared spectrophotometers for fruit dry matter assessment. Journal of Near Infrared Spectroscopy 2017; 25: 267-277. DOI: 10.1177/0967033517725530.

92. Norris K. The Algorithm for Norris Gap Derivative http://www.impublications.com/discus/messages/5/237.html?1172691604 (2004, accessed 08/28/2018 2018).

93. Norris KH. More on the Factors Affecting Diffuse Transmittance and Reflectance Spectra. NIR news 2002; 13: 8-11. DOI: 10.1255/nirn.664.

94. Ritchie GE and Mark H. Investigating NIR Transmittance Measurements through the Use of the Norris Regression (NR) Algorithm: Part 1: How Do We Come to "Norris Regression"? NIR news 2002; 13: 4-6. DOI: https://doi.org/10.1255/nirn.644.

95. Ritchie GE and Mark H. Investigating NIR Transmittance Measurements through the Use of the Norris Regression (NR) Algorithm: Part 2: Theoretical Considerations. NIR news 2002; 13: 3-5. DOI: https://doi.org/10.1255/nirn.653.

96. The algortihm for Norris Gap derivatives?, http://www.impublications.com/discus/messages/5/237.html?1172691604 (2007, accessed 01/13/2019 2019).

97. FOSS/NIRSystems. Vision and NSAS User Manual. In: FOSS/NIRSystems, (ed.). Silver Spring, MD, USA.

98. Hruschka W. SPANUSER.DOC Manual for Spectral Analysis Programs (SPAN). In: Laboratory UDoAARSIaS, (ed.). 1998.

99. Dardenne P. Calibration Transfer in near Infrared Spectroscopy. NIR news 2017; 13: 3-7. DOI: $10.1255 /$ nirn.668.

100. Hopkins DW. Revisiting the Norris Derivative Quotient Math in Regression. NIR news 2016; 27: 23-28. DOI: 10.1255/nirn.1643.

101. Hopkins DW. What is a Norris Derivative? NIR news 2017; 12: 3-5. DOI: 10.1255/nirn.611.

102. Bazar G, Kovacs Z and Tsenkova R. Evaluating Spectral Signals to Identify Spectral Error. PLoS One 2016; 11: e0146249. 2016/01/06. DOI: 10.1371/journal.pone.0146249. 103. Hayes CJ, Walsh KB and Greensill CV. Improving Calibration Transfer between Shortwave near Infrared Silicon Photodiode Array Instruments. Journal of Near Infrared Spectroscopy 2016; 24: 59-68. DOI: 10.1255/jnirs.1194.

104. C. Hayes KW, R. Lerud. Calibration transfer between short wave near infrared photodiode array instruments. NIR2017. Copenhagen, DE2017.

105. Fearn T. Standardisation and Calibration Transfer for near Infrared Instruments: A Review. Journal of Near Infrared Spectroscopy 2001; 9: 229-244.

106. Andrew A and Fearn T. Transfer by orthogonal projection: making near-infrared calibrations robust to between-instrument variation. Chemometrics and Intelligent Laboratory Systems 2004; 72: 51-56. DOI: 10.1016/j.chemolab.2004.02.004. 
107. Soldado A, Fearn T, Martinez-Fernandez A, et al. The transfer of NIR calibrations for undried grass silage from the laboratory to on-site instruments: comparison of two approaches. Talanta 2013; 105: 8-14. 2013/04/20. DOI: 10.1016/j.talanta.2012.11.028. 108. Igne B, Roger J-M, Roussel S, et al. Improving the transfer of near infrared prediction models by orthogonal methods. Chemometrics and Intelligent Laboratory Systems 2009; 99: 57-65. DOI: 10.1016/j.chemolab.2009.07.007.

109. Roggo Y, Duponchel L, Noe B, et al. Sucrose Content Determination of Sugar Beets by near Infrared Reflectance Spectroscopy. Comparison of Calibration Methods and Calibration Transfer. Journal of Near Infrared Spectroscopy 2017; 10: 137-150. DOI: 10.1255/jnirs.330.

110. Westerhaus MO. Improving Repeatability of Nir Calibrations across Instruments. In: Third international conference on Near Infrared Spectroscopy (ed Bartiaux-Thill RBaN), Brussels, Belgium, 1990, p.671.

111. Saranwong S and Kawano S. A Simple Method of Instrument Standardisation for a near Infrared Sorting Machine: The Utilisation of Average Spectra as Input Vectors. Journal of Near Infrared Spectroscopy 2017; 12: 359-365. DOI: 10.1255/jnirs.444. 112. Greensill CV and Walsh KB. A remote acceptance probe and illumination configuration for spectral assessment of internal attributes of intact fruit. Measurement Science and Technology 2000; 11: 1674-1684. DOI: 10.1088/0957-0233/11/12/304.

113. Guthrie JA, Walsh KB, Reid DJ, et al. Assessment of internal quality attributes of mandarin fruit. 1. NIR calibration model development. Australian Journal of Agricultural Research 2005; 56. DOI: 10.1071/ar04257.

114. Subedi PP and Walsh KB. Assessment of sugar and starch in intact banana and mango fruit by SWNIR spectroscopy. Postharvest Biology and Technology 2011; 62: 238245. DOI: 10.1016/j.postharvbio.2011.06.014.

115. Reyns P, Missotten B, Ramon $\mathrm{H}$, et al. A Review of Combine Sensors for Precision Farming. Precision Agriculture 2002; 3: 169-182. DOI: $10.1023 / a: 1013823603735$.

116. Ting VJ, Soukoulis $C$, Silcock $P$, et al. In vitro and in vivo flavor release from intact and fresh-cut apple in relation with genetic, textural, and physicochemical parameters. $J$ Food Sci 2012; 77: C1226-1233. 2012/10/13. DOI: 10.1111/j.1750-3841.2012.02947.x. 117. Yahia EM. Apple Flavor. Horticultural Reviews. John Wiley \& Sons, Inc., pp.197234. 
Appendix A - Supplemental Information Chapter 2

\section{Supplementary Information}

\section{Optimizing the Performance of a Commercial Electrochemical Ethylene}

\section{Gas Sensor}

Ryan M. Lerud ${ }^{a^{*}}$, Drew S. Beseau ${ }^{\mathrm{b}}$, Cameron M. Hale ${ }^{\mathrm{b}}$, Charles Noll ${ }^{\mathrm{b}}$, Shankar B. Rananavare ${ }^{\mathrm{a}, \mathrm{b}}$

${ }^{a}$ Department of Physics, ${ }^{b}$ Department of Chemistry, Portland State University. Portland OR 97206

*Corresponding author email: LERUD@PDX.EDU

Preparation of Ethephon/KCl pellets: A mixture of $0.324 \mathrm{~g}$ of ethephon with $9.000 \mathrm{~g}$ of $\mathrm{KCl}$ was prepared. $\mathrm{KCl}$ acts as an inert bulk diluent. The ethephon $/ \mathrm{KCl}$ mixture was dissolved in $250 \mathrm{~mL} \mathrm{2:1} \mathrm{ratio} \mathrm{of} \mathrm{deionized} \mathrm{(DI)} \mathrm{water} \mathrm{and} \mathrm{anhydrous} \mathrm{EtOH} \mathrm{(v:v).} \mathrm{It} \mathrm{was}$ dried on a hot plate with a magnetic stirrer at a solution temperature of $80^{\circ} \mathrm{C}$ and an initial stir speed of 600 RPM. During the final drying stages, the mixture was stirred using a glass stir rod replacing the magnetic stir bar to ensure the loose powdery solid had minimal clumping. The bulk material is assumed to be homogeneous and can be weighed out to form pellets using standard IR pellet press. The above procedure results in some loss of ethephon due to thermal degradation. Therefore, the weight of the pellets to releases fixed concentration of $\mathrm{C}_{2} \mathrm{H}_{4}$ was calibrated against $\mathrm{GC}$ and Electrochemical sensors. The effective concentration of Ethephon in pellets was constant yielding a reproducible fixed $\mathrm{C}_{2} \mathrm{H}_{4}$ concentration over a year. 
The schematic outline of F-900 electrochemical sensor (Felix Instruments, USA) appears below in Figure S-1.

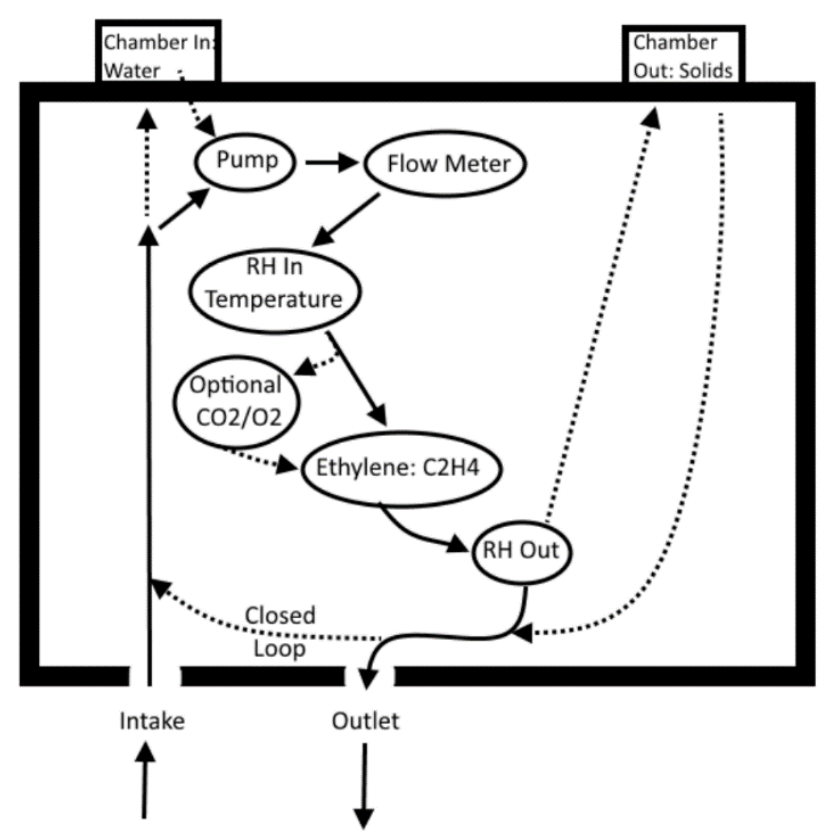

Figure S-1 Flow path diagram of the F-900 ethylene analyzer (Image taken from F-900 user manual). Dashed lines indicate optional flow path capabilities such as; additional $\mathrm{CO}_{2}$ sensor, additional $\mathrm{O}_{2}$ sensor, switches to enable/disable the polar VOC trap, switches to enable/disable onboard $\mathrm{KMnO}_{4}$, and switches to enable/disable internal closed loop. The input chamber which normally housed polar VOC trap (water) was replaced with the phosphate buffer solution of ethephon for field calibration of the electrochemical sensor with $\mathrm{C}_{2} \mathrm{H}_{4}$. While during the trap regeneration cycle, exhaust from the sensor was redirected into the output chamber containing 
$\mathrm{KMnO}_{4}$. During regeneration cycle (9-10 minutes for apple headspace), polar volatile components released from aqueous trap are oxidized to $\mathrm{CO}_{2}$.

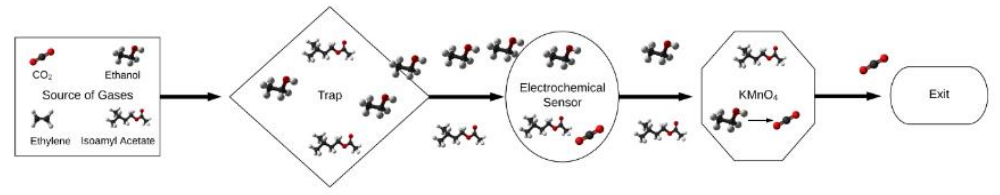

Figure S-2 A schematic illustration of the operation of a gas trap for interfering compounds such as acetates, alcohols. $\mathrm{CO}_{2}$ and ethylene pass through; ethanol is absorbed, and esters such as isoamyl acetate are expected to be partially trapped. $\mathrm{KMnO}_{4}$ solution oxidizes organic compounds to $\mathrm{CO}_{2}$ and $\mathrm{H}_{2} \mathrm{O}$.

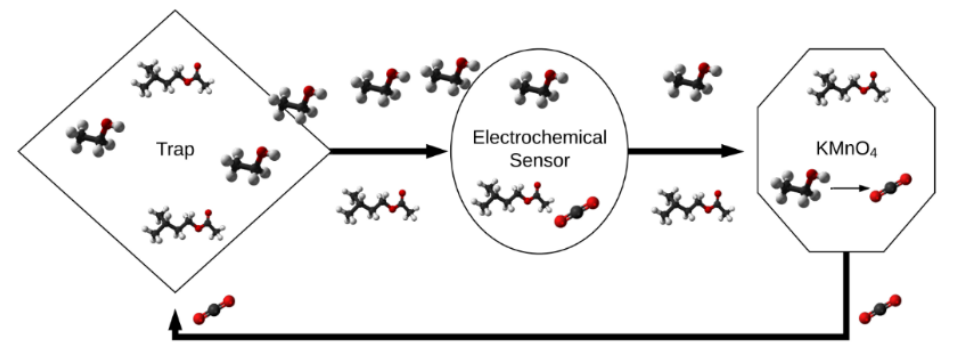

Figure S-3 The closed loop for regenerating the aqueous gas trap. The output chamber of F-900 contains $\mathrm{KMnO}_{4}$ pellets. The interfering species as well as species trapped in trap are removed by passing the gas from the output chamber through electrochemical and chemical oxidation steps as indicated. 


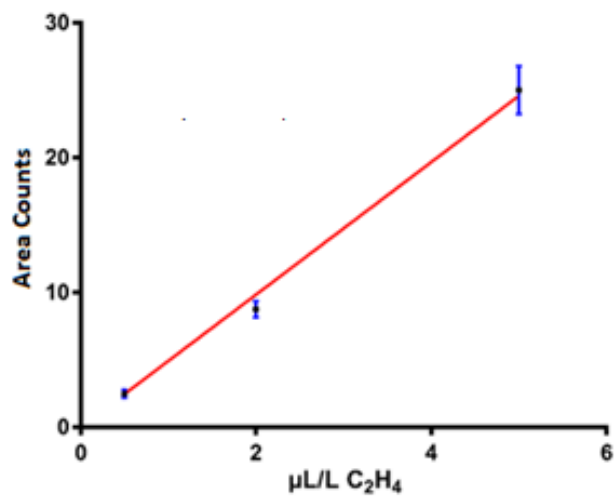

Figure S-4 Calibration curve for Ethylene determined from GC-FID detector. The peak area appearing at 2-minute elution point is determined numerically by integration, after applying a linear baseline correction. The slope and the intercepts are $0.004 \pm 0.001,-0.00 \pm 0.27$. 
Analysis of GC-MS data for changes in composition of polar VOCs before and after the input trap

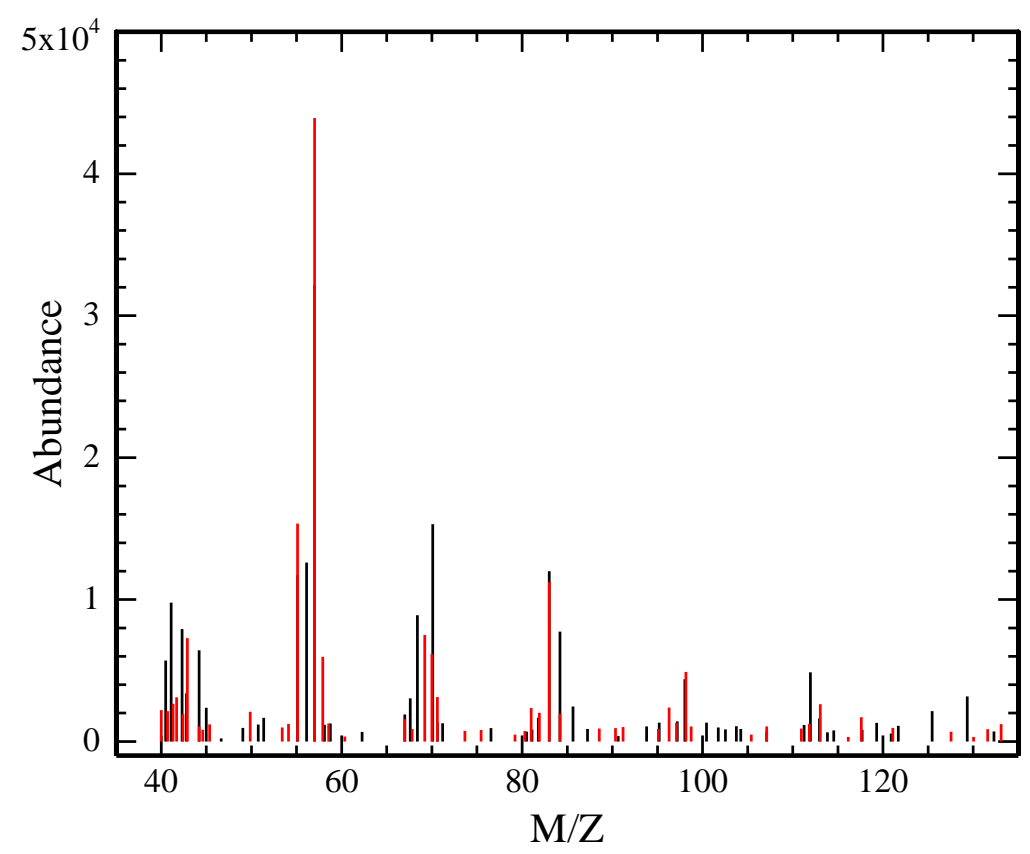

Figure S-5 MS Spectra from the headspace. Prior to (black) and after (red) vertical lines show relative abundance of mass fragments. 
Table S-1 Parent peaks that were lost after insertion of the polar trap are shown below.

\begin{tabular}{lll}
\hline $\mathbf{M} / \mathbf{Z}$ & Relative Abundance & Potential Compound \\
\hline 40.5 & 5701 & C3H3 \\
41.1 & 9775 & C3H5 \\
44.2 & 6432 & Acetaldehyde(44.01 \\
56.1 & 12599 & Propanal (58.8) \\
68.4 & 8891 & C3H5 \\
70.1 & 15315 & C5H10, Butanal (72), butanol \\
84.2 & 7743 & C6H12, 2-ethylbutanol \\
111.95 & 4868 & heptanol \\
100.45 & 1330 & Hexanal, hexanol \\
153.85 & 796 & -- \\
\hline
\end{tabular}

Table S-1 displays a partial list of mass fragments and their relative change in abundance before and after the trap. Heavier mass fragments are listed in Table S-2. As expected polar watersoluble components such as propanol, butanol, hexanol and heptanol are lost. Prior extensive studies of apple headspace ${ }^{47,116}$ have shown that the mass fragments at M/Z ratio of 70,84 correspond to $\mathrm{C}_{5} \mathrm{H}_{11}{ }^{+}$arising from the breakdown of Alcohol, ester fragments and well-known ${ }^{117}$ cis-3hexenol/ pentenal/alcohol/ester fragments respectively. The higher molecular components $>150$ have been ascribed to various terpenes/terpenoids ${ }^{117}$. 


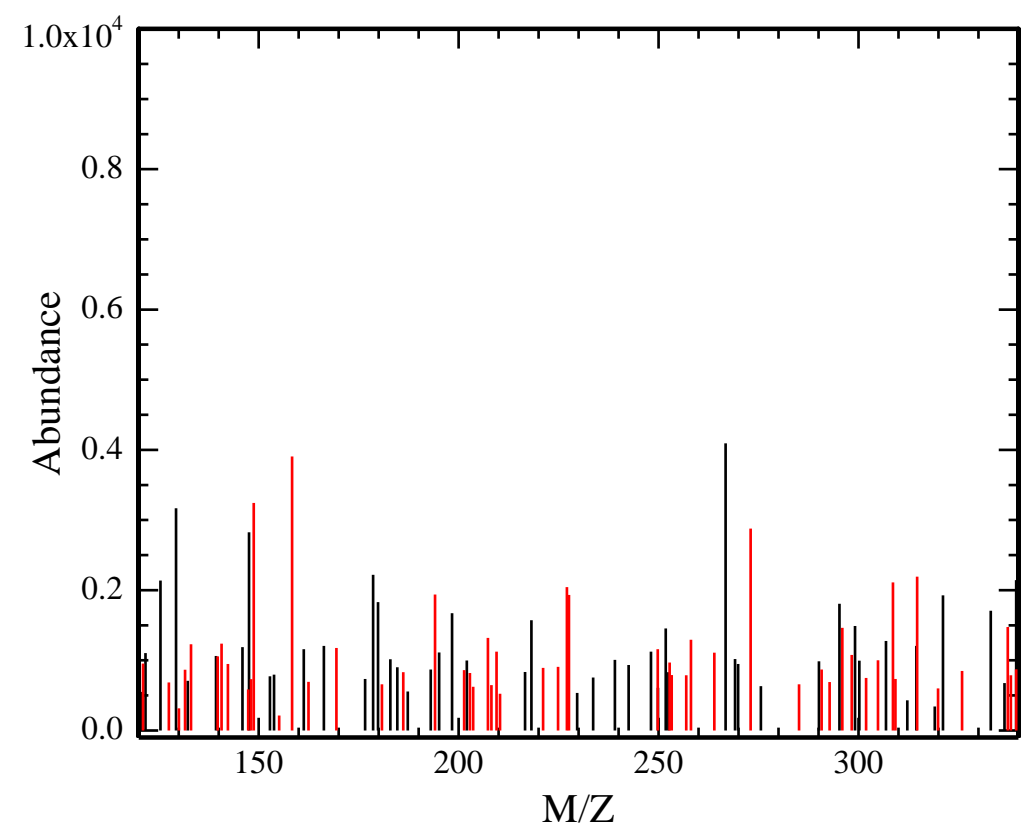

Figure S-6 MS Spectra from the headspace for mass fragments 100-350M/Z. Prior to (black) and after (red) vertical lines show relative abundance of mass fragments.

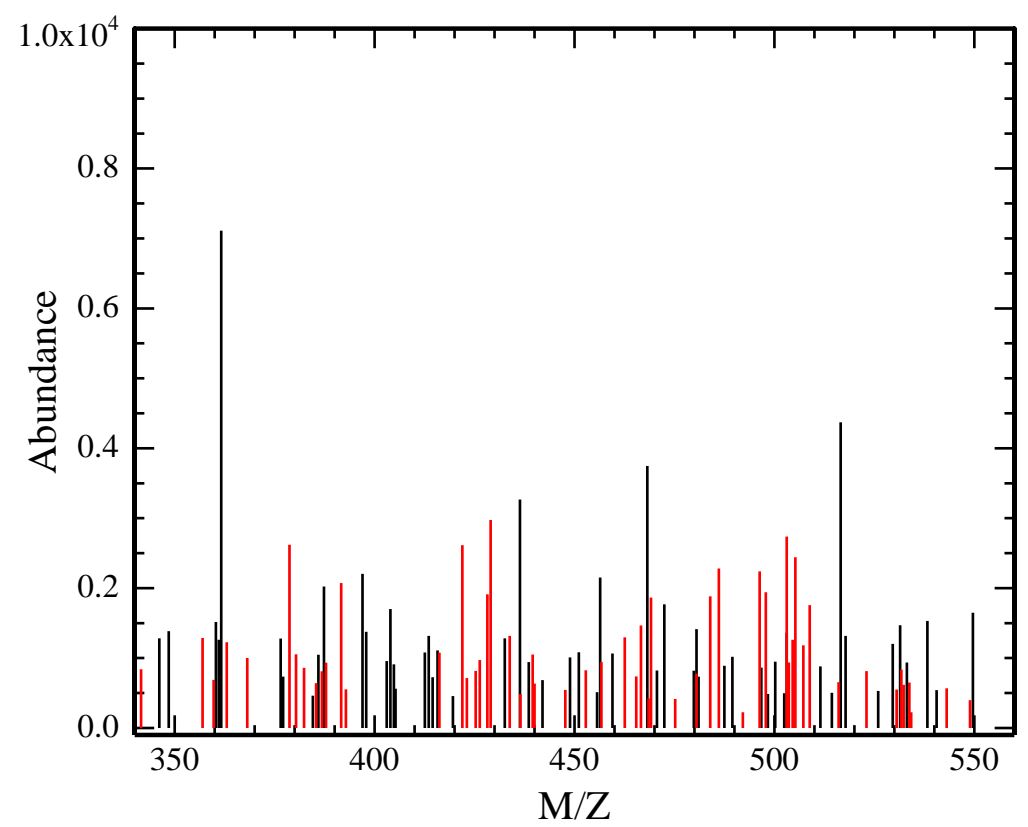

Figure S-7 MS Spectra from the headspace for mass fragments 350-550 M/Z. Prior to (black) and after (red) vertical lines show relative abundance of mass fragments. 
Table S-2 Heavier mass fragments and their relative abundance before and after the trap.

\begin{tabular}{lll}
\hline M/Z & Relative Abundance & Potential Compound \\
\hline 125.45 & 2138 & -- \\
129.35 & 3168 & 2-METHYLBUTYL ACETATE \\
178.65 & 2221 & Fragmentation of 2,3-Dihydro farnesyl acetate \\
266.8 & 4094 & $2,3-$ Dihydro farnesyl acetate \\
321.15 & 1927 & -- \\
339.45 & 2145 & -- \\
346.2 & 1282 & -- \\
348.55 & 1384 & -- \\
361.65 & 7112 & -- \\
386 & 1047 & -- \\
387.35 & 2024 & -- \\
404.85 & 910 & -- \\
436.4 & 3267 & -- \\
468.25 & 3748 & -- \\
516.6 & 4370 & -- \\
549.65 & 1649 & -- \\
\hline
\end{tabular}

\section{Equation S-1}

Ethylene Production Rate $=($ Ethylene ppmv * Flow Rate $) /($ Sample Mass $)$

$(1 \mu \mathrm{L} / \mathrm{L} * 6 \mathrm{~L} / \mathrm{hr}) /(1.65 \mathrm{~kg})=>$ ethylene production rate of $\sim 3.6 \mu \mathrm{L}$ per $\mathrm{kg} \cdot \mathrm{hr}$ 
Appendix B - Calibration transfer between short wave near infrared photodiode array instruments

C. Hayes ${ }^{a}$, K. Walsh ${ }^{b}$ and R. Lerud ${ }^{c}$

${ }^{a}$ Central Queensland University, Rockhampton, QLD, 4702, Australia. E-mail:

c.hayes@cqu.edu.au

${ }^{b}$ Central Queensland University, Rockhampton, QLD, 4702, Australia. E-mail:

k.walsh@cqu.edu.au

'Felix Instruments, Camas, WA, 98607, USA. E-mail: rlerud@felixinstruments.com

Transfer methods were compared for the porting of partial least squares models for intact mango dry matter content between short wave near infrared silicon photodiode array instruments. Methods included bias adjustment using average difference spectrum, new pixelto-wavelength assignments, piecewise direct standardisation (PDS), global models, model updating (MU) and combinations of these. Best results $\left(R^{2}>0.84\right.$ and bias $\left.<0.2\right)$ were obtained by PDS using the same variety of fruit in calibration and transfer sets. The use of an apple spectra transfer set was also successful, if the wavelength accuracy of the slave unit(s) is satisfactory. Alternatively, a field practical solution that gave acceptable prediction results involved development of a global model across units or model updating by inclusion of spectra of the new population, using reference values estimated using the master unit.

\section{Introduction}

Spectrometers employing silicon photodiode array (PDA) detectors operating over the short wave NIR (780-1000 $\mathrm{nm}$ ) are attractive for their low cost and relative ruggedness. However units can vary in terms of wavelength accuracy and photometric response, impacting on the success of use of a model transfer between units. Any shift in the wavelength scale will create prediction errors when a model created on one unit is used with another unit, especially when there is high model weighting on spectral regions with high slopes (see e.g. Fearn1). A wavelength dependant photometric response difference between units will result in spectra of different shapes, and thus also impact model transfer. 


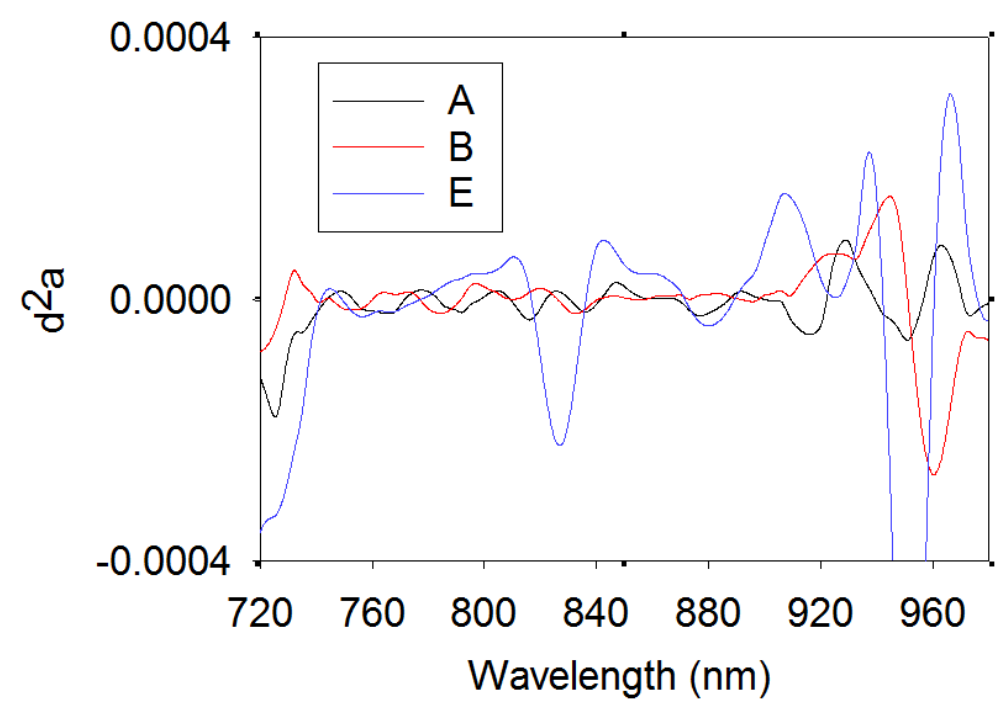

Figure 1 Difference second derivative of absorbance spectra (slave minus master) of a mango fruit for three instruments

Calibration models can be developed for each device, but this is inefficient. Model transfer between instruments can be approached in three ways (Fearn ${ }^{1}$, Andrew and Fearn ${ }^{2}$, Soldado et al. ${ }^{3}$, Igne et al. ${ }^{4}$ ): (i) by making of a model that transfers without requiring standardisation, e.g. using spectral pre-treatments, selecting wavelengths ranges that exhibit spectral 'stability', or by including data from multiple instruments in the calibration set; (ii) adjusting the model output such that it works on other instruments, e.g. simple slope and bias correction; (iii) correcting spectra from slave instruments to appear as they were acquired on master instrument, or (iv) transfer by orthogonal projection (TOP), with removal of spectral differences orthogonal to the calibration model.

The third approach in calibration transfer, of adjusting spectra from slave instruments to appear as master instrument spectra, has been addressed by a range of techniques. The spectral correction methods of spectral slope/bias correction (SSBC) and spectral bias correction5, involve wavelength by wavelength simple linear regressions of spectra from each instrument. Other techniques, as reviewed by Fearn1, include direct standardization (DS), piecewise direct standardization (PDS), double window piecewise direction standardization (DWPDS), orthogonal signal correction (OSC), finite impulse response (FIR) and wavelet transform (WT).

Other approaches achieve an 'implicit' orthogonalization, such as the 'repeatability' (REP) file concept ${ }^{6}$. In this method, difference spectra (of the same sample scanned on multiple instruments) are scaled proportional to the ratio of the number of samples in the REP file and calibration set, then assigned an attribute value of zero and included in the calibration set. In a similar approach, Saranwong and Kawano 7 proposed a method for transfer of a partial least 
squares (PLS) calibration on apple total soluble solids (TSS) between two Foss NIRSystems 6500 spectrometers, in which the difference in the average spectra (second derivative absorbance) of a group of samples is used to adjust the slave instrument spectra by simple subtraction. This method effects a bias correction (difference spectrum multiplied by model coefficients) and cannot improve the SEP (bias corrected RMSEP) of the transferred model. In the case presented, the method was effective, with the resulting bias at the same level as the validation results on the master instrument.

There are few reports on calibration transfer work involving silicon PDA based spectrometers. Greensill et al. ${ }^{8,9}$ compared the performance of the seven techniques mentioned above and a model updating (MU) technique based on use of Kennard-Stone selected representative spectra. WT and MU proved to be the best methods, decreasing the RMSEP from 7.03 to $0.21 \%$ TSS, with very little difference in the RMSEP of the two techniques, although PDS and DWPDS methods also performed well. Hayes et. al. ${ }^{10}$ investigated the impact of wavelength accuracy on PLS model performance for predicting apple TSS for SWNIR Si PDA based instruments and the benefit of improvement in wavelength calibration in concert with transfer routines for the porting of models between instruments. The instruments used employ a Zeiss MMS1 spectrometer with an interactance geometry. Wavelength assignment errors of up to $2.3 \mathrm{~nm}$ the range used in the PLS regressions on TSS were noted in some units. Both SEP and bias increased with inaccuracies in wavelength assignments. The transfer methodologies of PDS, TOP, MU and the difference spectrum adjustment (DSA) of Saranwong and Kawano7 were trialled. The DSA method combined with new wavelength assignments and model updating gave results comparable to the performance of the master instrument and to models directly developed on the slave instruments $\left(r^{2}=0.95\right.$, SEP $_{-b}=0.47$ and bias $=-0.03 \% \mathrm{TSS}$, for a population of mean $=$ 14.45 and $\mathrm{SD}=1.64 \% \mathrm{w} / \mathrm{v})$. Although comparable, the DSA method combined with new wavelength assignments and model updating was preferred over PDS due to ease of implementation.

An issue of practical importance is that of the selection of the updating or transfer set. Bouveresse and Massart ${ }^{11}$, Fearn ${ }^{1}$, and de Noord ${ }^{12}$ suggest that the standardisation samples used should cover the same spectral intensity range as the model/prediction set, and those which cover a larger spectral range generally lead to poor results. Thus the use of 'real' or similar samples with the same spectral features as associated with samples to be predicted is advocated. However, the definition of 'similar' requires consideration. For fruit, for example, does the PDS transfer set need to be based on the same fruit commodity or indeed the same fruit cultivar as that used in the model? Previous studies have used a subset of a calibration set for transfer.

Blanco et al. ${ }^{13}$ asserted that the wavelength accuracy is of more importance than absorbance response differences for model transfer across diode array UV-Vis spectrometers. The reverse is generally true for scanning monochromator NIR spectrometers. The wavelength to pixel assignments of PDA units are commonly calculated from a third order polynomial based on peak positions of mercury/argon lamp spectra. Peak assignment differences of $0.26 \mathrm{~nm}$ for a Si PDA 
with approximately $3.3 \mathrm{~nm}$ pixel dispersion have been reported ${ }^{14}$. Given a wavelength resolution (full width half maximum) of around $10 \mathrm{~nm}$ in these instruments, and as SWNIR features are wide, a wavelength accuracy of around $1 \mathrm{~nm}$ would appear appropriate for matching of instruments. However, this is not so, e.g. Kaur et al. ${ }^{15}$ observed significant variation in Si PDA instrument performance (RMSEP) for (fruit dry matter content) models developed using the same wavelength range, a result ascribed to the variation in wavelength to pixel assignments of the two units.

The current study compares transfer methods for the porting of models between instruments, expanding an earlier consideration 10 to include the methods of global modelling, global model updating and wavelength reassignment using non-biological samples. Our work is focused on the application of SWNIRS to internal quality of fruit, so this study was framed by work with a spectrometer system and data set relevant to that application.

\section{Materials and Methods}

Fruit spectra were acquired on six F-750 instruments (Felix Instruments, Camas, WA, USA) and destructively sampled for oven dry matter (\%DM). These instruments employ a Zeiss MMS1-Nir enhanced spectrometer in an interactance geometry. This geometry involves a probe receiving light to the MMS1 fiber optic placed in front of a halogen lamp mounted in a parabolic reflector, such that a shadow is cast on the sample, and the detected light is largely derived from this shadowed area. The MMS1 in five of the units employed a S8381 Si PDA detector (Hamamatsu Photonics K.K., Hamamatsu City, Shizuoka, Japan), while the older sixth unit employed a S4874 Si PDA detector (Hamamatsu Photonics K.K.). The change in array used in the MMS1 was made by Zeiss to reduce signal carryover between successive readouts and improve read-out time. The pixel to wavelength assignment in this unit differed to that of other units.

Two spectra were collected from two locations on each fruit. After spectra collection, $2 \mathrm{~cm}$ diameter by $1 \mathrm{~cm}$ deep cores of flesh were taken, skin removed, and dry matter content (DM) assessed gravimetrically following drying at $65^{\circ} \mathrm{C}$ to constant weight.

The following fruit sets were scanned using all instruments. A population of mango cultivar Kensington Pride fruit ( $n=232, \mu=13.7, \sigma=1.6 \% D M$ ) were used for calibration, with separate sets fruit of the same cultivar but different harvest dates used in validation ( $n=100, \mu=13.8, \sigma=1.5$ $\% D M)$, and all transfer methods ( $n=100, \mu=14.4, \sigma=1.3 \% D M)$. For further PDS transfer functions, spectra were collected of: (i) a set of cultivar Calypso fruit ( $n=280, \mu=16.6, \sigma=1.0 \% D M$ ); (ii) R2E2 fruit ( $n=300, \mu=14.7, \sigma=1.4 \% D M)$ and (iii) apple fruit ( $n=176, \mu=12.9, \sigma=1.3 \% D M)$.

As HgAr lamp spectra could not be collected without disassembly of the instruments, the master instrument was arbitrarily defined as having "correct" pixel-to-wavelength assignments. Pixelto-wavelength assignments for the $695-1014 \mathrm{~nm}$ range were generated using a fourth order polynomial fit to wavelength peaks of spectra of polypropylene, using wavelength assignments from the master unit. 
PLS regression models were developed using MATLab R2014a (MathWorks Inc., Natick, MA, USA) with PLS toolbox 7.3 (Eigenvector Research Inc., Wenatchee, WA, USA), using mean centered 9 point Savitzky-Golay second derivative absorbance data, interpolated to $3 \mathrm{~nm}$ steps, and the wavelength range 732-936 nm. The choice of number of PLS factors was based on minimisation of RMSECV, with 7 factors chosen in most cases.

The PLS DM model developed for the master unit was used 'direct' in prediction using unaltered spectra of other units, with results compared to those for several instrument standardisation techniques involving either spectral correction (PDS, DSA), global models or model updating (Table 1). For the DSA technique, the mean difference spectra was calculated using spectra of the Kensington Pride fruit transfer set, and used in adjustment of all validation set spectra. This was also combined with the new pixel-to-wavelength assignments, as described above. PDS was performed using the four different transfer sets listed above.

Several MU and global model approaches were trialed using the Kensington Pride transfer set: (i) a global model with all spectra of the calibration sets of units $A$ to $D$ (with $E$ included only on its own); (ii) same as (i) with increased PLS factors (iii) a master unit based model, updated with spectra from the transfer sets of units A to D, (iv) same as (iii) with increased PLS factors, (v) model based on spectra of the master unit updated with spectra from the transfer set of the respective slave unit, (vi) a model based on spectra of the master unit, updated with spectra from the transfer set of the slave unit only, but predicted DM values from the master unit, (vii) slave unit based model, using spectra from the slave transfer set and predicted DM values from the master unit. The last two methods seek to improve practicality of implementation by avoiding the need for reference method analyses.

\section{Results and Discussion}

Spectra of the same sample differed between units, with peak positions varying in wavelength assignment by up to $4 \mathrm{~nm}$ in the case of the older unit (unit E), as revealed in difference spectra (difference in the second derivative of absorbance spectra of polypropylene between master and slave units, Fig. 1). This result is similar to that reported by Kaur et al. ${ }^{15}$ for the same spectrometer type. The variation in difference spectra between units $A$ to $E$ and the master unit is ascribed to the wavelength assignment issues, although detector wavelength sensitivity could also contribute to this observation. Due to this, when attempting methods (i) - (iv) above, spectra from unit $E$ was only included when developing models for use on unit $E$,

The performance of models created on one unit and used in prediction of spectra collected on another unit was impacted both in terms of $\mathrm{R}^{2}$ (and corresponding SEP - data not shown) and bias (Table 1. 'Direct' results). The DSA method improved bias for all slave unit predictions, although bias magnitude $>0.5$ occurred in two cases, without affecting $R^{2}$. In these two cases, the difference spectra, which was calculated using the transfer set, did not match the difference of the validation sets. The new pixel to wavelength assignment combined with DSA improved $\mathrm{R}^{2}$ of unit $E$, as expected due to the discrepancy in wavelength calibration of this unit. All other transfer methods trialed were successful in decreasing bias (Table 1). 
Table 1. (A) DM prediction statistics for models developed on a given unit using spectra of a calibration set of Kensington Pride fruit from respective units, used in prediction of an independent population of fruit; (B) DM prediction statistics for the calibration model transferred (using various methods) from the F-750 unit master to slave units A, B, C, D and E. For a given unit, best results are bolded ( $R^{2}$ values within 0.2 units, bias of 0.3 or less).

\begin{tabular}{lcccccc}
\hline $\mathbf{R}^{2}$ & Master & $\mathbf{A}$ & $\mathbf{B}$ & $\mathbf{C}$ & $\mathbf{D}$ & $\mathbf{E}$ \\
\hline A. Calibration on single unit & 0.90 & 0.88 & 0.84 & 0.89 & 0.90 & 0.91 \\
\hline B. Transfer between units & & & & & & \\
Direct & 0.90 & 0.78 & 0.53 & $\mathbf{0 . 9 0}$ & 0.36 & 0.57 \\
DSA & $\mathrm{N} / \mathrm{A}$ & 0.78 & 0.53 & $\mathbf{0 . 9 0}$ & 0.36 & 0.57 \\
DSA + wavelength & $\mathrm{N} / \mathrm{A}$ & 0.82 & 0.51 & 0.86 & 0.56 & 0.82 \\
reassignment & & & & & & \\
PDS & $\mathrm{N} / \mathrm{A}$ & $\mathbf{0 . 8 7}$ & $\mathbf{0 . 8 4}$ & 0.89 & $\mathbf{0 . 8 8}$ & $\mathbf{0 . 9 1}$ \\
PDS(Calypso) & $\mathrm{N} / \mathrm{A}$ & $\mathrm{N} / \mathrm{A}$ & 0.82 & 0.88 & 0.85 & 0.89 \\
PDS(R2E2) & $\mathrm{N} / \mathrm{A}$ & $\mathrm{N} / \mathrm{A}$ & 0.41 & 0.82 & $\mathbf{0 . 8 6}$ & $\mathbf{0 . 9 2}$ \\
$\begin{array}{l}\text { PDS (Apple) } \\
\text { Global }\end{array}$ & $\mathrm{N} / \mathrm{A}$ & $\mathbf{0 . 8 9}$ & $\mathbf{0 . 8 3}$ & 0.88 & $\mathbf{0 . 8 7}$ & 0.65 \\
Global (10 PCs) & 0.83 & 0.84 & 0.75 & 0.85 & 0.79 & 0.75 \\
$\begin{array}{l}\text { Master model, global updating } \\
\text { Master model, global updating }\end{array}$ & $\mathbf{0 . 9 0}$ & $\mathbf{0 . 8 9}$ & $\mathbf{0 . 8 5}$ & $\mathbf{0 . 9 2}$ & $\mathbf{0 . 8 6}$ & 0.79 \\
$\begin{array}{l}\text { (10 PCs) } \\
\text { Individual model updating }\end{array}$ & $\mathbf{0 . 9 1}$ & 0.86 & 0.82 & 0.88 & 0.85 & 0.77 \\
$\begin{array}{l}\text { Individual model updating - } \\
\text { master predicted DM } \\
\text { Individual models using }\end{array}$ & $\mathbf{0 . 9 0}$ & 0.85 & $\mathbf{0 . 8 6}$ & $\mathbf{0 . 9 2}$ & $\mathbf{0 . 8 7}$ & 0.81 \\
master predicted DM & 0.89 & 0.85 & 0.82 & 0.84 & 0.84 & 0.81 \\
\hline
\end{tabular}


Table 2. (A) DM prediction statistics for models developed on a given unit using spectra of a calibration set of Kensington Pride fruit from respective units, used in prediction of an independent population of fruit; (B) DM prediction statistics for the calibration model transferred (using various methods) from the $F-750$ unit master to slave units $A, B, C, D$ and $E$. For a given unit, best results are bolded ( $R^{2}$ values within 0.2 units, bias of 0.3 or less).

\begin{tabular}{|c|c|c|c|c|c|c|}
\hline Bias & Master & $\mathbf{A}$ & B & C & D & $E$ \\
\hline A. Calibration on single unit & 0.01 & -0.14 & 0.16 & 0.12 & 0.05 & -0.02 \\
\hline \multicolumn{7}{|l|}{ B. Transfer between units } \\
\hline Direct & 0.01 & 1.94 & -0.26 & -2.81 & -1.68 & $\begin{array}{c}- \\
17.64\end{array}$ \\
\hline DSA & $\mathrm{N} / \mathrm{A}$ & -0.37 & -0.57 & 0.22 & -0.63 & 0.03 \\
\hline $\begin{array}{l}\text { DSA + wavelength } \\
\text { reassignment }\end{array}$ & $\mathrm{N} / \mathrm{A}$ & 0.18 & -0.27 & 0.38 & -0.17 & 0.01 \\
\hline PDS & $\mathrm{N} / \mathrm{A}$ & 0.01 & 0.09 & 0.14 & 0.20 & -0.01 \\
\hline PDS(Calypso) & $\mathrm{N} / \mathrm{A}$ & $\mathrm{N} / \mathrm{A}$ & 0.04 & -0.07 & -0.09 & -0.66 \\
\hline PDS(R2E2) & $\mathrm{N} / \mathrm{A}$ & $\mathrm{N} / \mathrm{A}$ & 0.40 & -0.87 & -0.03 & -0.30 \\
\hline PDS (Apple) & $\mathrm{N} / \mathrm{A}$ & -0.20 & 0.11 & 0.12 & 0.14 & -1.20 \\
\hline Global & -0.03 & 0.30 & 0.42 & -0.08 & -0.13 & 0.38 \\
\hline Global (10 PCs) & 0.02 & -0.04 & 0.19 & 0.07 & -0.16 & 0.37 \\
\hline $\begin{array}{l}\text { Master model, global } \\
\text { updating }\end{array}$ & 0.14 & 0.14 & 0.36 & -0.08 & -0.07 & 0.43 \\
\hline $\begin{array}{l}\text { Master model, global } \\
\text { updating (10 PCs) }\end{array}$ & -0.03 & -0.15 & 0.08 & 0.07 & -0.30 & 0.23 \\
\hline Individual model updating & -0.09 & -0.42 & -0.22 & -0.05 & -0.21 & 0.02 \\
\hline $\begin{array}{l}\text { Individual model updating - } \\
\text { master predicted DM }\end{array}$ & 0.01 & -0.22 & -0.07 & 0.10 & -0.07 & 0.12 \\
\hline $\begin{array}{l}\text { Individual models using } \\
\text { master predicted DM }\end{array}$ & -0.01 & 0.14 & 0.14 & 0.07 & -0.03 & -0.24 \\
\hline
\end{tabular}

Prediction $\mathrm{R}^{2}$ was most consistently improved using PDS (based on a transfer set of the same variety of fruit as the calibration model), the Global (10 factors) and the Master model global updating (10 factors) approaches. However, the use of the same fruit variety for the PDS transfer set as used for the calibration set can be impractical due to seasonal availability of fruit. PDS using a related variety (Calypso) was successful across all units, however use of fruit of the R2E2 variety for the transfer set proved unsuccessful on some units. Surprising, the use of apple spectra as the PDS training set proved quite successful, except on unit $\mathrm{E}$. This latter result is believed to be due to the drastic difference in wavelength alignment of the units. 
Except for unit $E$, a global model using spectra from all instruments gave satisfactory performance when the number of PLS factors was increased to 10, allowing the PLS calibration to model for instrument difference.

The performance of models based on individual unit model updating (model based on master calibration set plus transfer set from slave unit) was also comparable to that of PDS based models. Interestingly, use of DM values estimated by the master instrument (with no wet chemistry performed) for the transfer set (spectra collected on slave unit), gave acceptable performance. This approach has the advantage of being able to be implemented rapidly in field situations.

\section{Conclusion}

Best results ( $\mathrm{R}^{2}$ and bias) were obtained using PDS using the same variety of fruit in calibration and transfer sets. This method is impractical due to seasonal availability of fruit. If the wavelength accuracy of the slave unit(s) is satisfactory, apples could be used to transfer the mango calibrations all year round (i.e. 'out of mango season'). A global model across units or model updating, using reference values estimated using the master unit can be utilized in field situations.

\section{Appendix B References}

1. T. Fearn, "Standardisation and Calibration Transfer for near Infrared Instruments: A Review", J. Near Infrared Spectrosc 9, 229 (2001). http://dx.doi.org/10.1255/jnirs.309

2. A. Andrew and T. Fearn, "Transfer by Orthogonal Projection: Making near-Infrared Calibrations Robust to between-Instrument Variation", Chemometrics and Intelligent Laboratory Systems 72, 51 (2004). http://dx.doi.org/10.1016/j.chemolab.2004.02.004

3. A. Soldado, T. Fearn, A. Martínez-Fernández and B. de la Roza-Delgado, "The Transfer of Nir Calibrations for Undried Grass Silage from the Laboratory to on-Site Instruments: Comparison of Two Approaches", Talanta 105, 8 (2013). http://dx.doi.org/10.1016/j.talanta.2012.11.028

4. B. Igne, J.-M. Roger, S. Roussel, V. Bellon-Maurel and C.R. Hurburgh, "Improving the Transfer of near Infrared Prediction Models by Orthogonal Methods", Chemometrics and Intelligent Laboratory Systems 99, 57 (2009). http://dx.doi.org/10.1016/j.chemolab.2009.07.007

5. Y. Roggo, L. Duponchel, B. Noe and J.P. Huvenne, "Sucrose Content Determination of Sugar Beets by near Infrared Reflectance Spectroscopy. Comparison of Calibration Methods and Calibration Transfer", J. Near Infrared Spectrosc 10, 137 (2002). http://dx.doi.org/10.1255/jnirs.330

6. M.O. Westerhaus, "Improving Repeatability of Nir Calibrations across Instruments," in Third international conference on Near Infrared Spectroscopy, R. Biston and N. Bartiaux-Thill, Eds., Brussels, Belgium, pp. 671 (1990). 
7. S. Saranwong and S. Kawano, "A Simple Method of Instrument Standardisation for a near Infrared Sorting Machine: The Utilisation of Average Spectra as Input Vectors", J. Near Infrared Spectrosc 12, 359 (2004). http://dx.doi.org/10.1255/jnirs.444

8. C.V. Greensill and K.B. Walsh, "Calibration Transfer between Miniature Photodiode Array-Based Spectrometers in the near Infrared Assessment of Mandarin Soluble Solids Content", J. Near Infrared Spectrosc 10, 27 (2002). http://dx.doi.org/10.1255/jnirs.318

9. C.V. Greensill, P.J. Wolfs, C.H. Spiegelman and K.B. Walsh, "Calibration Transfer between Pda-Based Nir Spectrometers in the Nir Assessment of Melon Soluble Solids Content", Applied Spectroscopy 55, 647 (2001). http://dx.doi.org/10.1366/0003702011952280

10. C. Hayes, K. Walsh and C. Greensill, "Improving Calibration Transfer between Shortwave near Infrared Silicon Photodiode Array Instruments", J. Near Infrared Spectrosc 24, 59 (2016). http://dx.doi.org/10.1255/jnirs.1194

11. E. Bouveresse, D.L. Massart and P. Dardenne, "Modified Algorithm for Standardization of near-Infrared Spectrometric Instruments", Analytical Chemistry 67, 1381 (1995). http://dx.doi.org/10.1021/ac00104a013

12. O.E. de Noord, "Multivariate Calibration Standardization", Chemometrics and Intelligent Laboratory Systems 25, 85 (1994). http://dx.doi.org/10.1016/0169-7439(94)85037-2

13. M. Blanco, J. Coello, H. Iturriaga, S. Maspoch and E. Rovira, "Wavelength Calibration Transfer between Diode Array Uv-Visible Spectrophotometers", Applied Spectroscopy 49, 593 (1995). http://dx.doi.org/10.1366/0003702953964084

14. Zeiss, "Test Cetificate." Carl Zeiss Jena GmbH, Jena, (2002).

15. H. Kaur, R. Künnemeyer and A. McGlone, "Comparison of Hand-Held near Infrared Spectrophotometers for Fruit Dry Matter Assessment", J. Near Infrared Spectrosc 25, 267 (2017). http://dx.doi.org/10.1177/0967033517725530 